\title{
DIXMIER TRACES AND SOME APPLICATIONS IN NONCOMMUTATIVE GEOMETRY
}

\author{
Alan L. Carey \\ Mathematical Sciences Institute \\ Australian National University \\ Canberra, ACT. 0200, AUSTRALIA \\ e-mail: acarey@maths.anu.edu.au
}

\author{
Fyodor A. Sukochev \\ School of Informatics and Engineering \\ Flinders University \\ Bedford Park S.A 5042 AUSTRALIA \\ e-mail: sukochev@infoeng.flinders.edu.au
}

\section{Contents}

1. Introduction 3

2. Preliminaries: spaces and functionals 6

2.1. Marcinkiewicz function and sequence spaces 6

2.2. Singular symmetric functionals on Marcinkiewicz spaces. $\quad 7$

2.3. Symmetric operator spaces and functionals. $\quad 8$

3. General facts about symmetric functionals. 10

4. Preliminaries on dilation and translation invariant states. 12

5. Concrete constructions of singular symmetric functionals. 16

5.1. Dixmier traces 16

5.2. Connes-Dixmier traces 19

5.3. Rearrangement invariant functionals and singular traces. 20

6. Class of measurable elements. 20

7. Norming properties of Dixmier and Connes-Dixmier functionals 23

8. Fredholm modules and spectral triples 25

8.1. Notation and definitions 25

8.2. Bounded versus unbounded 26

8.3. More on Semifinite Spectral Triples 26

8.4. Summability and Dimension 27

9. $\quad$ Spectral Flow 28

9.1. Spectral Flow Formulae 29 
9.2. Relation to Cyclic Cohomology 29

10. The Dixmier trace and residues of the zeta function 31

10.1. Preliminaries 31

10.2. The zeta function and the Dixmier trace 35

11. The heat semigroup formula 38

12. The case of $p>1 \quad 40$

13. Generalised Toeplitz operators and their index 41

14. Non-smooth foliations and pseudo-differential operators 43

15. The algebra of almost periodic functions. 44

15.1. Almost periodic pseudodifferential operators. 44

15.2. Almost periodic spectral triple $\quad 47$

16. Lesch's Index Theorem 48

17. The Hochschild Class of the Chern Character 51

18. Lidskii type formula for Dixmier traces 53

$\begin{array}{ll}\text { References } & 55\end{array}$ 


\section{INTRODUCTION}

The Dixmier trace $\tau_{\omega}$ arose from the problem of whether the algebra $B(\mathcal{H})$ of all bounded linear operators on a Hilbert space $\mathcal{H}$ had a unique non-trivial trace. Dixmier resolved this question in the negative in 1966 in a note in Comptes Rendus [41]. To construct the trace he used an invariant mean $\omega$ on the solvable ' $a x+b$ ' group. His trace vanishes on the ideal of trace class operators and hence is completely disjoint from the usual trace. It is also non-normal.

Applications of the Dixmier trace to classical geometry are facilitated by a remarkable result [33] relating the trace to the Wodzicki [93] residue for pseudo-differential operators on a closed manifold. The latter was introduced independently by Adler [1], Manin [76] in the one dimensional case and developed further by Wodzicki [93] and Guillemin [62] in higher dimensions. The intriguing and most useful property of the Wodzicki residue apart from its computability in examples is the fact that it makes sense for pseudo-differential operators of arbitrary order. Moreover it is the unique trace on the pseudo-differential operators which extends the Dixmier trace on operators of order $\leqslant$ minus the dimension of the underlying manifold [33].

Both the Dixmier trace and the Wodzicki residue play important roles in noncommutative geometry and its applications [31,57]. In particular, the Dixmier trace $\tau_{\omega}$ is an appropriate noncommutative analogue of integration on a compact $n$-dimensional Riemannian spin manifold $M$, more exactly

$$
\tau_{\omega}\left(f(x)|D|^{-n}\right)=\frac{1}{(2 \pi)^{n} n} 2^{[n / 2]} \Omega_{n-1} \int_{M} f(x) d \mathrm{vol},
$$

where $f$ is a smooth function on $M, D$ is the Dirac operator on $M, \Omega_{n-1}$ is the volume of $n-1$-dimensional unit sphere and $d$ vol is the Riemannian volume form. The usefulness of the Dixmier trace is extended by the results of Connes [31] which relates it to residues of zeta functions.

Important applications which we do not have the space to include are to the theory of gravitation, classical field theory and particle physics. The former is well covered in the book [57] and has its origins in the relationship of the Wodzicki residue of powers of the Dirac operator to the Einstein-Hilbert action [33]. Equally we do not try to cover other material in the books [31] and [57] which describe several interesting physical applications of the Dixmier trace. We refer the reader to the survey [34] and the extensive literature on the application of noncommutative geometry to the 'standard model' of particle physics where, starting with an elegant expression for a noncommutative action principle expressed in terms of the Dixmier trace, Connes and Lott [39] and Connes [35] show how to derive from it the Euclidean version of the action for the standard model. Analogously, one may also give a noncommutative formulation of the Hamiltonian version of classical field theory again using the Dixmier trace [65]. Some background to these developments is provided by [67].

These applications to physics can be traced back to the research announcement [38] where a series of foundational results are described (some of these were later collected in [31]). After calculating the Dixmier trace for pseudodifferential operators Connes obtained the Yang-Mills and Polyakov actions from an action functional involving the Dixmier trace. The theorem on a residue formula for the Hochschild class of the Chern character, which we will describe in Section 17, was also announced. The next step appears in [37] where Connes introduced the 
axioms of noncommutative spin geometry for a noncommutative algebra $\mathcal{A}$. The very first axiom uses the Dixmier trace to introduce a noncommutative integration theory on $\mathcal{A}$ which is completely natural in view of (1). This point of view resurfaces in our discussion of Lesch's index theorem in Section 16 although we will not digress further to introduce the other axioms of Connes except to mention in Section 17 the role of the Hochschild class.

While these applications to physical theory form a motivational background they are not the focus of this article. Our aim is to give a unified and coherent account of some recent functional analytic advances in the theory of Dixmier traces. In addition to surveying these new results we also offer in some cases new proofs. Part of our motivation is to extend and clarify questions raised by [31] Chapter IV. Specifically we characterise the class of measurable operators defined in [31], explain the role of the Cesaro mean in Connes' version of Dixmier traces (called ConnesDixmier traces here) and give a complete analysis of zeta function formulae for the Dixmier trace. Most importantly, however, our whole treatment is within the framework of 'semifinite spectral triples' which we explain in Section 8. This notion arises when one extends the theory in [31], which deals with subalgebras of the bounded operators on a separable Hilbert space equipped with the standard trace, to the situation where the von Neumann algebra of bounded operators is replaced by a general semifinite von Neumann algebra equipped with some faithful normal semifinite trace ( $\mathrm{cf}[7]$ ). As a result of the extra effort needed to handle this level of generality it is possible to find improvements even in the standard type $I$ theory of [31]. A number of authors have contributed to the development of this framework and new applications of Dixmier and more general singular traces [2], [3], [4], [8], [10], [32] [19], [20], [21], [22], [23], [24], [25], [47], [48], [58], [59], [68], [83], [61], [60], [91].

Our exposition is organized around three issues. The first issue dominates the early part of the article (culminating in Section 6) and gives the characterization of measurable operators in the sense of [31] Section IV.2. We approach the topic from the study of the general theory of singular symmetric functionals (this and its preliminaries occupy Sections 2 to 5). Our exposition is based on the approach articulated in [47, 25], which considers such traces as a special class of continuous linear functionals on the corresponding operator ideals. Many features of the theory may be well understood, even in the most trivial situation, when the von Neumann algebras in question are commutative. In this situation, the theory of Dixmier traces roughly corresponds to the theory of symmetric functionals on rearrangement invariant function spaces $[45,46,47]$ and allows an alternative treatment based on the methods drawn from real analysis.

In preparation for the rest of the paper we then introduce the notions of semifinite spectral triple, type $I I$ spectral flow and some notation for cyclic cohomology. This leads us into the second issue, occupying Sections 10 and 12, where we describe the expression of the Dixmier trace in terms of residues of zeta functions. The key observation, made in Section 4, that enables us to prove a considerable generalisation of Proposition IV.2.4 of [31] and also the measurability theorem of Section 6, is the existence of two kinds of Dixmier trace. One is associated with the multiplicative group of the positive reals and its invariant mean and the second, which is naturally associated with the zeta function, arises from the invariant mean of the additive group of the reals. The relationship between the two captures the formula for the Dixmier trace in terms of the zeta function. 
These results use the language of spectral triples and lead naturally to the third issue, namely some applications, which begin in Section 11. We give a proof of (an extension of) the heat semigroup formula of [31] (pp 563) for the Dixmier trace. We then give a formula for the index of generalised Toeplitz operators (Section 13), describe a special case of the Wodzicki residue formula of Benameur and Fack [10] for pseudodifferential operators along the leaves of a foliation and discuss in Section 15 a similar formula for pseudodifferential operators with almost periodic coefficients. The formula of Section 13 has, as a consequence, the index theorem of Lesch for Toeplitz operators with noncommutative symbol and this is described in Section 16. An extension of Theorem IV.2.8 of [31] on a residue formula for the Hochschild class of the Chern character of Fredholm modules is given in Section 17 (see also [10]).

Finally, following [8], we show how Lidskii's formula may be extended to Dixmier traces in the von Neumann setting in Section 18. In a series of corollaries we explain its relevance to the question of measurability. In [31], except for the case of pseudodifferential operators, measurability results are proved for positive operators. The approach of [8] allows one to address the problem of removing the positivity assumption.

We present below a short list of symbols and terminology used in this paper with the indication of the place where these symbols and notations are introduced:

- Symmetric and rearrangement invariant (r.i.) functionals and rearrangement invariant spaces $E(J)$ (Section 2, Definition 2.1);

- Marcinkiewicz spaces, $M(\psi), \mathcal{L}^{(1, \infty)}, \mathcal{L}^{(p, \infty)}$ (Section 2.1);

- Generalized singular value function $\mu_{(\cdot)}(x)$ (Section 4);

- Semifinite von Neumann algebra $\mathcal{N}$, faithful normal semifinite trace on $\mathcal{N}, \tau$. $\tau$ measurable operators $\widetilde{\mathcal{N}}$, the fully symmetric operator space associated to $(\mathcal{N}, \tau)$ and Banach function space $E$ is denoted $E(\mathcal{N}, \tau)$ (Section 2.3);

- Operator Marcinkiewicz spaces $M(\psi)(\mathcal{N}, \tau)$ (Section 2.3);

- Symmetric functionals on a fully symmetric space $E=E(0, \infty): E_{\text {sym }}^{*}$ (Section 3.1);

- Sets of states $B L(\mathbb{R}), D\left(\mathbb{R}_{+}^{*}\right), B L\left(\mathbb{R}_{+}\right)$(Section 4 );

- Dixmier traces $\tau_{\omega}$ (Definitions 5.1,5.4), traces $F_{\mathbb{L}}, F_{\mathbb{L}}$ (Section 5.1);

- Connes-Dixmier traces $\tau_{\omega}$ with $\omega \in C D\left(\mathbb{R}_{+}^{*}\right)$ (Section 5.2);

- Measurable operators (Definitions 6.1 and 6.2);

- Spectral triples $(\mathcal{A}, \mathcal{H}, \mathcal{D})$, grading operator $\Gamma, \mathcal{I}$-summability (Definition 8.1 );

- $Q C^{k}$ semifinite spectral triples (Definition 8.6);

- Spectral dimension of $(\mathcal{A}, \mathcal{H}, \mathcal{D})$ (Definition 8.7);

- Spectral flow $s f\left(\left\{F_{t}\right\}\right), s f\left(D, u D u^{*}\right)$ (Section 9);

- (b,B)-cochain, (b,B)-cocycle, $\left(b^{T}, B^{T}\right)$-chain, $\left(b^{T}, B^{T}\right)$-cycle (Section 9$)$;

- Zeta functions $\zeta(s), \zeta_{A}(s)$ (Section 10.2);

- Bohr compactification $\mathbb{R}_{B}^{n}, C^{*}$-algebra $\mathcal{A} P\left(\mathbb{R}^{n}\right)$ (Section 15.1).

Acknowledgement The authors would like to thank N.A. Azamov and A.A. Sedaev, for comments, assistance and criticism. 


\section{PReliminaries: SPACES AND FUnCtionals}

Consider a Banach space $\left(E,\|\cdot\|_{E}\right)$ of real valued Lebesgue measurable functions (with identification $\lambda$ a.e.) on the interval $J=[0, \infty)$ or else on $J=\mathbb{N}$. Let $x^{*}$ denote the non-increasing, right-continuous rearrangement of $|x|$ given by

$$
x^{*}(t)=\inf \{s \geqslant 0 \mid \lambda(\{|x|>s\}) \leqslant t\}, t>0,
$$

where $\lambda$ denotes Lebesgue measure. Then $E$ will be called rearrangement invariant if

(i). $E$ is an ideal lattice, that is if $y \in E$, and $x$ is any measurable function on $J$ with $0 \leqslant|x| \leqslant|y|$, then $x \in E$ and $\|x\|_{E} \leqslant\|y\|_{E}$;

(ii). if $y \in E$ and if $x$ is any measurable function on $J$ with $x^{*}=y^{*}$, then $x \in E$ and $\|x\|_{E}=\|y\|_{E}$.

In the case $J=\mathbb{N}$, it is convenient to identify $x^{*}$ with the rearrangement of the sequence $|x|=\left\{\left|x_{n}\right|\right\}_{n=1}^{\infty}$ in the descending order. For basic properties of rearrangement invariant $(=$ r.i. $)$ spaces we refer to the monographs [69], [71], [72]. We note that for any r.i. space $E=E(J)$ the following continuous embeddings hold

$$
L_{1} \cap L_{\infty}(J) \subseteq E \subseteq L_{1}+L_{\infty}(J) .
$$

The r.i. space $E$ is said to be fully symmetric Banach space if it has the additional property that if $y \in E$ and $L_{1}+L_{\infty}(J) \ni x \prec \prec y$, then $x \in E$ and $\|x\|_{E} \leqslant\|y\|_{E}$. Here, $x \prec \prec y$ denotes submajorization in the sense of Hardy-Littlewood-Pólya:

$$
\int_{0}^{t} x^{*}(s) d s \leqslant \int_{0}^{t} y^{*}(s) d s, \quad \forall t>0 .
$$

A classical example of non-separable fully symmetric function and sequence spaces $E(J)$ is given by Marcinkiewicz spaces.

2.1. Marcinkiewicz function and sequence spaces. Let $\Omega$ denote the set of concave functions $\psi:[0, \infty) \rightarrow[0, \infty)$ such that $\lim _{t \rightarrow 0^{+}} \psi(t)=0$ and $\lim _{t \rightarrow \infty} \psi(t)=\infty$. Important functions belonging to $\Omega$ include $t, \log (1+t), t^{\alpha}$ and $(\log (1+t))^{\alpha}$ for $0<\alpha<1$. Let $\psi \in \Omega$. Define the weighted mean function

$$
a(x, t)=\frac{1}{\psi(t)} \int_{0}^{t} x^{*}(s) d s \quad t>0
$$

and denote by $M(\psi)$ the Marcinkiewicz space of measurable functions $x$ on $[0, \infty)$ such that

$$
\|x\|_{M(\psi)}:=\sup _{t>0} a(x, t)=\|a(x, \cdot)\|_{\infty}<\infty .
$$

The definition of the Marcinkiewicz sequence space $\left(m(\psi),\|x\|_{m(\psi)}\right)$ is similar,

$$
m(\psi)=\left\{x=\left\{x_{n}\right\}_{n=1}^{\infty}:\|x\|_{m(\psi)}:=\sup _{N \geqslant 1} \frac{1}{\psi(N)} \sum_{n=1}^{N} x_{n}^{*}<\infty\right\} .
$$


The norm closure of $M(\psi) \cap L^{1}\left([0, \infty)\right.$ ) (respectively, of $\left.\ell_{1}=\ell_{1}(\mathbb{N})\right)$ in $M(\psi)$ (respectively, in $m(\psi)$ ) is denoted by $M_{1}(\psi)$ (respectively, $m_{1}(\psi)$ ). For every $\psi \in \Omega$, we have $M_{1}(\psi) \neq M(\psi)$. The Banach spaces $\left(M(\psi),\|\cdot\|_{M(\psi)}\right),\left(m(\psi),\|\cdot\|_{m(\psi)}\right),\left(M_{1}(\psi),\|\cdot\|_{M(\psi)}\right),\left(m_{1}(\psi),\|\cdot\|_{m(\psi)}\right)$ are examples of fully symmetric spaces [71], [69].

Let $M_{+}(\psi)$ (respectively, $\left.m_{+}(\psi)\right)$ denote the set of positive functions of $M(\psi)$ (respectively, $m(\psi))$. For every $x \in M(\psi)$, we write $x=x_{+}-x_{-}$, where $x_{+}:=x \chi_{\{t: x(t)>0\}}$ and $x_{-}:=$ $x-x_{+}$. The spaces

$$
\mathcal{L}^{(1, \infty)}:=M(\log (1+t)) \cap L_{\infty} \quad \text { and } \quad \mathcal{L}^{(p, \infty)}:=M\left(t^{1-\frac{1}{p}}\right) \cap L_{\infty}, \quad 1<p<\infty
$$

play very important part in the sequel. Note that these spaces are still Marcinkiewicz spaces. Indeed, $\mathcal{L}^{(1, \infty)}$ (respectively, $\mathcal{L}^{(p, \infty)}, p>1$ ) may be identified with the space $M\left(\psi_{1}\right)$ (respectively $\left.M\left(\psi_{p}\right), p>1\right)$, where

$$
\psi_{1}(t)=\left\{\begin{array}{ll}
t \cdot \log 2, & 0 \leqslant t \leqslant 1 \\
\log (1+t), & 1 \leqslant t<\infty
\end{array},\right.
$$

respectively,

$$
\psi_{p}(t)=\left\{\begin{array}{ll}
t, & 0 \leqslant t \leqslant 1 \\
t^{1-\frac{1}{p}}, & 1 \leqslant t<\infty
\end{array} .\right.
$$

The (Marcinkiewicz) norm given by formula $(2)$ on the space $\mathcal{L}^{(p, \infty)}$ is denoted by $\|\cdot\|_{(p, \infty)}$, $1 \leqslant p<\infty$.

\subsection{Singular symmetric functionals on Marcinkiewicz spaces.}

Definition 2.1 (cf. [47], Definition 2.1). A positive functional $f \in M(\psi)^{*}$ is said to be symmetric (respectively, r.i.) if $f(x) \leqslant f(y)$ for all $x, y \in M_{+}(\psi)$ such that $x \prec \prec y$ (respectively, $x^{*}=y^{*}$ ). Such a functional is said to be supported at infinity (or singular) if $f(|x|)=0$ for all $x \in M_{1}(\psi)$ (equivalently, $f\left(x^{*} \chi_{[0, s]}\right)=0$, for every $x \in M(\psi)$ and the indicator function $\chi_{[0, s]}$ of the interval $[0, s]$ for all $\left.s>0\right)$.

The following theorem completely characterizes Marcinkiewicz spaces admitting non-trivial symmetric functionals.

Theorem $2.2([47,45,46])$. If $\psi \in \Omega$, then

(i). a non-zero symmetric functional on $M(\psi)$ (respectively, $m(\psi))$ supported at infinity exists if and only if

$$
\liminf _{t \rightarrow \infty} \frac{\psi(2 t)}{\psi(t)}=1
$$

(ii). a non-zero symmetric functional on $M(\psi)$ supported at zero exists if and only if

$$
\liminf _{t \downarrow 0} \frac{\psi(2 t)}{\psi(t)}=1 .
$$


Thus, for example, $\left(\mathcal{L}^{(1, \infty)}\right)_{\text {sym }, \infty}^{*} \neq\{0\}$, whereas $\left(\mathcal{L}^{(p, \infty)}\right)_{\text {sym, } \infty}^{*}=\{0\}$, for all $1<p<\infty$. The conditions (3) and (4) admit the following geometric interpretation. Let us denote by $N(\psi)$ the norm closure in $M(\psi)$ of the (order) ideal

$$
N(\psi)^{0}:=\left\{f \in M(\psi): f^{*}(\cdot) \leqslant k \psi^{\prime}\left(\frac{\dot{b}}{k}\right) \text { for some } k \in \mathbb{N}\right\} .
$$

Clearly, $N(\psi)$ is a Banach function space (a subspace of $M(\psi)$ ) and is rearrangement invariant. Assuming (for simplicity) that $\psi$ is linear in a neighbourhood of 0 , the space $N(\psi)$ is fully symmetric (and thus coincides with $M(\psi)$ ) if and only if (3) fails. In other words, $M(\psi)_{\text {sym }, \infty}^{*} \neq\{0\}$ if and only if $N(\psi) \neq M(\psi)$. For this and other geometric interpretations of conditions (3) and (4) we refer the reader to [13],[69, II.5.7] and [86]. For various constructions of singular symmetric functionals on $M(\psi)$ (and more generally on fully symmetric spaces and their non-commutative counterparts) we refer to [47], [45], [46]. Constructions relevant to our main topic will be reviewed below, in Section 5 .

Our focus on symmetric functionals supported at infinity is explained by the numerous applications of their non-commutative counterparts in non-commutative geometry. Noncommutative analogues of symmetric functionals supported at zero can be thought of as "Dixmier traces associated with von Neumann algebras of type $I I_{1}$ " and have not found any applications to date.

2.3. Symmetric operator spaces and functionals. Here, we extend the ideas of the previous sections to the setting of (noncommutative) spaces of measurable operators. We denote by $\mathcal{N}$ a semifinite von Neumann algebra on the Hilbert space $\mathcal{H}$, with a fixed faithful and normal semifinite trace $\tau$. We shall be mainly concerned with $\tau(1)=\infty$, where 1 is the identity in $\mathcal{N}$. A linear operator $x: \operatorname{dom}(x) \rightarrow \mathcal{H}$, with domain $\operatorname{dom}(x) \subseteq \mathcal{H}$, is called affiliated with $\mathcal{N}$ if $u x=x u$ for all unitary $u$ in the commutant $\mathcal{N}^{\prime}$ of $\mathcal{N}$. The closed and densely defined operator $x$, affiliated with $\mathcal{N}$, is called $\tau$-measurable if for every $\epsilon>0$ there exists an orthogonal projection $p \in \mathcal{N}$ such the $p(\mathcal{H}) \subseteq \operatorname{dom}(x)$ and $\tau(1-p)<\epsilon$. The set of all $\tau$-measurable operators is denoted $\widetilde{\mathcal{N}}$.

We next recall the notion of generalized singular value function $[53,51]$. Given a self-adjoint operator $x$ in $\mathcal{H}$, we denote by $e^{x}(\cdot)$ the spectral measure of $x$. Now assume that $x$ is $\tau$ measurable. Then $e^{|x|}(B) \in \mathcal{N}$ for all Borel sets $B \subseteq \mathbb{R}$, and there exists $s>0$ such that $\tau\left(e^{|x|}(s, \infty)\right)<\infty$. For $t \geqslant 0$, we define

$$
\mu_{t}(x)=\inf \left\{s \geqslant 0: \tau\left(e^{|x|}(s, \infty)\right) \leqslant t\right\} .
$$

The function $\mu(x):[0, \infty) \rightarrow[0, \infty]$ is called the generalized singular value function (or decreasing rearrangement) of $x$; note that $\mu_{t}(x)<\infty$ for all $t>0$. For the basic properties of this singular value function we refer the reader to [53].

If we consider $\mathcal{N}=L_{\infty}([0, \infty), m)$, where $m$ denotes Lebesgue measure on $[0, \infty)$, as an abelian von Neumann algebra acting via multiplication on the Hilbert space $\mathcal{H}=$ $L^{2}([0, \infty), m)$, with the trace given by integration with respect to $m$, it is easy to see that the set of all $\tau$-measurable operators affiliated with $\mathcal{N}$ consists of all measurable functions on $[0, \infty)$ which are bounded except on a set of finite measure, and that the generalized singular value function $\mu(f)$ is precisely the decreasing rearrangement $f^{*}$. 
If $\mathcal{N}=\mathcal{L}(\mathcal{H})$ (respectively, $\ell_{\infty}(\mathbb{N})$ ) and $\tau$ is the standard trace $\operatorname{Tr}$ (respectively, the counting measure on $\mathbb{N}$ ), then it is not difficult to see that $\widetilde{\mathcal{N}}=\mathcal{N}$. In this case, $x \in \mathcal{N}$ is compact if and only if $\lim _{t \rightarrow \infty} \mu_{t}(x)=0$; moreover,

$$
\mu_{n}(x)=\mu_{t}(x), \quad t \in[n, n+1), \quad n=0,1,2, \ldots,
$$

and the sequence $\left\{\mu_{n}(x)\right\}_{n=0}^{\infty}$ is just the sequence of eigenvalues of $|x|$ in non-increasing order and counted according to multiplicity.

Given a semifinite von Neumann algebra $(\mathcal{N}, \tau)$ and a fully symmetric Banach function space $\left(E,\|\cdot\|_{E}\right)$ on $([0, \infty), m)$, we define the corresponding non-commutative space $E(\mathcal{N}, \tau)$ by setting

$$
E(\mathcal{N}, \tau)=\{x \in \widetilde{\mathcal{N}}: \mu(x) \in E\} .
$$

Equipped with the norm $\|x\|_{E(\mathcal{N}, \tau)}:=\|\mu(x)\|_{E}$, the space $\left(E(\mathcal{N}, \tau),\|\cdot\|_{E(\mathcal{N}, \tau)}\right)$ is a Banach space and is called the (non-commutative) fully symmetric operator space associated with $(\mathcal{N}, \tau)$ corresponding to $\left(E,\|\cdot\|_{E}\right)$. If $\mathcal{N}=\ell_{\infty}(\mathbb{N})$, then the space $E(\mathcal{N}, \tau)$ is simply the (fully) symmetric sequence space $\ell_{E}$, which may be viewed as the linear span in $E$ of the vectors $e_{n}=\chi_{[n-1, n)}, n \geqslant 1$ (see e.g. [71]). In the case $(\mathcal{N}, \tau)=(\mathcal{L}(\mathcal{H}), T r)$, we denote $E(\mathcal{N}, \tau)$ simply by $E(\mathcal{H})$. Note, that the latter space coincides with the (symmetricallynormed) ideal of compact operators on $\mathcal{H}$ associated with (symmetric) sequence space $\ell_{E}$ (see e.g $[56])$.

We shall be mostly concerned with fully symmetric operator spaces $E(\mathcal{N}, \tau)$ and $E(\mathcal{H})$ when $E=M(\psi)$, in particular, when $E=\mathcal{L}^{(p, \infty)}, 1 \leqslant p<\infty$. We refer to the spaces $M(\psi)(\mathcal{N}, \tau)$ as to operator Marcinkiewicz spaces. Sometimes, for brevity, we shall omit the symbols $(\mathcal{N}, \tau)$ and $\mathcal{H}$ from the notations and this should not cause a confusion. $18]$.

Further references to the theory of fully symmetric operator spaces can be found in $[47,17$,

Definition 2.3. A linear functional $\varphi \in E(\mathcal{N}, \tau)^{*}$ is called symmetric (respectively, r.i.) if $\varphi$ is positive, (that is, $\varphi(x) \geqslant 0$ whenever $0 \leqslant x \in E(\mathcal{N}, \tau)$ ) and $\varphi(x) \leqslant \varphi(y)$ whenever $\mu(x) \prec \prec \mu(y)$ (respectively, $\varphi(x)=\varphi(y)$ whenever $x, y \geqslant 0$ and $\mu(x)=\mu(y))$.

For a given $x \in \tilde{\mathcal{N}}$, the set $\Omega(x)=\{y \in \widetilde{\mathcal{N}}: y \prec \prec x\}$ is called the orbit of the operator $x$. If $x \in L_{1}(\mathcal{N}, \tau)+\mathcal{N}$, then the set $\Omega(x)$ is conveniently described in terms of absolute contractions. Denote by $\Sigma$ the set of all linear operators $T: L_{1}(\mathcal{N}, \tau)+\mathcal{N} \rightarrow L_{1}(\mathcal{N}, \tau)+\mathcal{N}$ such that $T(a) \in L_{1}(\mathcal{N}, \tau)$ (respectively, $\mathcal{N}$ ) if $a \in L_{1}(\mathcal{N}, \tau)$ (respectively, $\mathcal{N}$ ) and such that $\|T\|_{L_{1}(\mathcal{N}, \tau) \rightarrow L_{1}(\mathcal{N}, \tau)} \leqslant 1,\|T\|_{\mathcal{N} \rightarrow \mathcal{N}} \leqslant 1$. It follows from [44] that $y \prec \prec x, x \in L_{1}(\mathcal{N}, \tau)+\mathcal{N}$, $y \in \widetilde{\mathcal{N}}$ if and only if there exists $T \in \Sigma$ such that $T(x)=y$. Thus,

$$
\Omega(x)=\{T x: T \in \Sigma\} .
$$

If $E(\mathcal{N}, \tau)$ is a fully symmetric operator space, we have $\Omega(x) \subseteq E(\mathcal{N}, \tau)$ for every $x \in E(\mathcal{N}, \tau)$ and therefore a bounded positive linear functional $\varphi$ on $E(\mathcal{N}, \tau)$ is symmetric if and only if $\varphi(|T x|) \leqslant \varphi(x)$ for every $T \in \Sigma$ and $0 \leqslant x \in E(\mathcal{N}, \tau)$.

Now we assume that $\alpha: \mathcal{N} \rightarrow \mathcal{N}$ is a $*$-automorphism which is in addition trace preserving, that is, $\tau(\alpha(a))=\tau(a)$ for all $0 \leqslant a \in \mathcal{N}$. It is easy to see that such an automorphism 
extends (uniquely) to a $*$-automorphism $\tilde{\alpha}: \widetilde{\mathcal{N}} \rightarrow \widetilde{\mathcal{N}}$, which is rearrangement preserving, that is, $\mu(\tilde{\alpha}(x))=\mu(x)$ for all $x \in \tilde{\mathcal{N}}$. Thus, we can view rearrangement invariant functionals on $E(\mathcal{N}, \tau)$ as positive functionals which are invariant with respect to the action of the group of all trace preserving $*$-automorphisms of $\mathcal{N}$, which is a subgroup of $\Sigma$. If $\mathcal{N}=\mathcal{L}(\mathcal{H})$, then every rearrangement invariant functional on $E(\mathcal{H})$ is simply a trace (i.e. unitary invariant positive functional on $\mathcal{L}(\mathcal{H})$ ), which extends to a continuous linear functional on $E(\mathcal{H})$. Clearly, every symmetric functional is rearrangement invariant. However, a priori, it is not clear (see [61]) whether there exists (for example on the ideal $\mathcal{L}^{(1, \infty)}(\mathcal{H})$ ) a rearrangement invariant singular functional, which is not necessarily symmetric, or whether there exists a trace on $\mathcal{L}^{(1, \infty)}(\mathcal{H})$, which does not coincide with a Dixmier trace (see Section 5 below). Very recently the first example of such a trace has appeared in [68]. In fact, if

$$
\lim _{t \rightarrow \infty} \frac{\psi(2 t)}{\psi(t)}=1
$$

then there exists a non-zero trace (or r.i. functional) on every operator Marcinkiewicz space $M(\psi)(\mathcal{N}, \tau)$ which vanishes on $N(\psi)(\mathcal{N}, \tau)$. In particular, if $\psi(t)=\log (1+t)$, then such a functional vanishes on every operator $0 \leqslant x \in \mathcal{L}^{(1, \infty)}(\mathcal{N}, \tau)$ with $\mu_{t}(x)=\frac{1}{t}$ (or, if $\mathcal{N}=B(\mathcal{H}$ ) then on every compact operator $0 \leqslant x \in \mathcal{L}^{(1, \infty)}(\mathcal{H})$, such that $\left.\mu_{n}(x)=\frac{1}{n}, n \geqslant 1\right)$. It is not clear yet whether r.i. functionals which are not symmetric exist on every $M(\psi)(\mathcal{N}, \tau)$ with $\psi$ satisfying condition (3).

\section{General facts about symmetric functionals.}

Let $E=E(0, \infty)$ be a fully symmetric space. By $E_{+}$we denote the set of all nonnegative functions from $E$ and by $E_{\text {sym }}^{*}$ the set of all symmetric functionals of $E$.

A positive linear functional $\varphi$ on $E$ is called normal (or order continuous), if from $f_{n} \downarrow 0$ it follows that $\varphi\left(f_{n}\right) \downarrow 0$.

Proposition 3.1. [47] If a functional $\varphi \in E_{\text {sym }}^{*}$ is order continuous, then $E \subset L^{1}[0, \infty)$, and $\varphi$ is proportional to the integral against the Lebesgue measure.

We define the dilation operator $D_{s}$ as in [69] by $D_{s} f(t)=f(t / s)$. Note that $D_{s}$ is a bounded operator on $E$ and $\left\|D_{s}\right\|_{E \rightarrow E} \leqslant \max \{1, s\}$, moreover $\left(D_{s} f\right)^{*}=D_{s} f^{*}$ for any function $f \in E$. The following result is established in [47, Proposition 2.3] under the assumption that $\varphi$ is symmetric, however the proof holds also for r.i. functionals.

Proposition 3.2. If $0 \leqslant \varphi \in E^{*}$ is rearrangement invariant, then $\varphi\left(D_{s} f\right)=s \varphi(f)$ for all $f \in E$ and $s>0$.

A positive $\varphi \in E^{*}$ is said to be singular, if from $0 \leqslant \varphi^{\prime} \leqslant \varphi, \varphi^{\prime} \in E^{*}, \varphi^{\prime}$ is order-continuous, it follows that $\varphi^{\prime}=0$.

Proposition 3.3. [47] (i) Every symmetric functional on $E$ can be uniquely decomposed into the sum of a normal functional and a singular symmetric functional. Moreover, the normal functional is zero unless $E \subseteq L^{1}[0, \infty)$.

(ii) Any singular symmetric functional can be uniquely decomposed into the sum of singular symmetric functionals, supported at zero and at infinity.

(iii) The set of symmetric functionals forms a lattice. 
The following result shows that every symmetric functional on $E$ admits a "natural extension" up to a symmetric functional on $E(\mathcal{N}, \tau)$ for every semifinite von Neumann algebra $(\mathcal{N}, \tau)$. By $E(\mathcal{N}, \tau)_{+}$we denote the set of all positive operators from $E(\mathcal{N}, \tau)$.

Theorem $3.4([47])$. Let $\varphi_{0} \in E_{\text {sym }}^{*}$. If $\varphi(x):=\varphi_{0}(\mu(x))$, for all $x \in E(\mathcal{N}, \tau)_{+}$, then $\varphi$ extends to a symmetric functional $0 \leqslant \varphi \in E(\mathcal{N}, \tau)^{*}$.

Proof. It clearly suffices to show that $\varphi$ is additive on $E(\mathcal{N}, \tau)_{+}$. Let $x, y \in E(\mathcal{N}, \tau)_{+}$. Since $\mu(x+y) \ll \mu(x)+\mu(y)\left(\left[53\right.\right.$, Theorem 4.4]), and since $\varphi_{0}$ is symmetric, it follows that

$$
\varphi(x+y)=\varphi_{0}(\mu(x+y)) \leqslant \varphi_{0}(\mu(x)+\mu(y))=\varphi(x)+\varphi(y) .
$$

To prove the converse inequality, we use the easily verified fact (see e.g. [58, Proposition 1.10]) that

$$
\int_{0}^{t} \mu_{s}(x) d s+\int_{0}^{t} \mu_{s}(y) d s \leqslant \int_{0}^{2 t} \mu_{s}(x+y) d s, \quad \forall t>0 .
$$

Observing that (6) is equivalent to the submajorization

$$
\mu(x)+\mu(y) \prec 2 D_{\frac{1}{2}} \mu(x+y),
$$

it follows from Proposition 3.2 that

$$
\begin{aligned}
\varphi(x)+\varphi(y) & =\varphi_{0}(\mu(x)+\mu(y)) \\
& \leqslant 2 \varphi_{0}\left(D_{\frac{1}{2}} \mu(x+y)\right)=\varphi_{0}(\mu(x+y))=\varphi(x+y) .
\end{aligned}
$$

Thus $\varphi$ is additive on $E(\mathcal{N}, \tau)_{+}$and this suffices to complete the proof of the Theorem.

Theorem 3.5. Let $(\mathcal{N}, \tau)$ be a semifinite von Neumann algebra without minimal projections, and let $E$ be a fully symmetric Banach function space on $[0, \infty)$. If $0 \leqslant \varphi \in E(\mathcal{N}, \tau)^{*}$ is a symmetric functional, then there exists a symmetric functional $0 \leqslant \varphi_{0} \in E^{*}$ such that $\varphi(x)=\varphi_{0}(\mu(x))$ for all $0 \leqslant x \in E(\mathcal{N}, \tau)$.

Proof. It is sufficient to show that there exists a symmetric functional $0 \leqslant \varphi_{0} \in E[0, \tau(1))^{*}$ satisfying $\varphi(x)=\varphi_{0}(\mu(x))$ for all $0 \leqslant x \in E(\mathcal{N}, \tau)$. Let $\mathcal{M}$ be the commutative von Neumann algebra $L_{\infty}[0, \tau(1))$, with trace given by integration. The algebra $\widetilde{\mathcal{M}}$ may be identified with the space of all measurable functions on $[0, \tau(1))$ which are bounded except on a set of finite measure. Since $\mathcal{M}$ does not contain any minimal projections, there exists a positive rearrangement-preserving algebra homomorphism $J: \widetilde{\mathcal{M}} \rightarrow \widetilde{\mathcal{N}}([18$, Lemma 4.1], [43, Theorem 3.5]). Let $0 \leqslant \varphi \in E(\mathcal{N}, \tau)^{*}$ be symmetric. For $f \in E[0, \tau(1))$, define $\varphi_{0}(f):=\varphi(J f)$. It is clear that $0 \leqslant \varphi_{0} \in E[0, \tau(1))^{*}$ is symmetric. Moreover, if $0 \leqslant x \in E(\mathcal{N}, \tau)$, then $\mu(J(\mu(x)))=\mu(x)$ and hence $\varphi(x)=\varphi(J(\mu(x)))=\varphi_{0}(\mu(x))$.

It is even easier to see that the correspondence between the sets $E_{\text {sym }}^{*}$ given in Theorems 3.4 and 3.5 also exists for the set of symmetric functionals $\left(\ell_{E}\right)_{\text {sym }}^{*}$ and $(E(\mathcal{H}))_{\text {sym }}^{*}$. Furthermore, as the following result shows there exists a simple connection also between the sets $\left(\ell_{E}\right)_{s y m}^{*}$ and $E_{\text {sym }}^{*}$.

Theorem 3.6 ([47]). Let $E$ be a fully symmetric Banach function space on $[0, \infty)$ and let $E(\mathcal{H})$ be the corresponding ideal of compact operators on infinite-dimensional Hilbert space $\mathcal{H}$. If $0 \leqslant \varphi \in E(\mathcal{N}, \tau)^{*}$ is a symmetric functional, then there exists $\varphi_{0} \in E_{\text {sym }}^{*}$ such that $\varphi(x)=\varphi_{0}(\mu(x))$ for all $x \in E(\mathcal{N}, \tau)_{+}$. 
Now, let us consider the following question naturally arising from Theorem 3.4 and suggested in [61]. Suppose that $E$ (respectively, $\ell_{E}$ ) is a fully symmetric function (respectively, sequence) space and $\varphi_{0}$ is a positive r.i. functional on $E$ (respectively, $\ell_{E}$ ). Is the functional

$$
\varphi(x):=\varphi_{0}(\mu(x))
$$

additive on $E(\mathcal{N}, \tau)$ (respectively, $E(\mathcal{H}))$ ? Very recently this question has been answered in the affirmative in [68], provided that $\mathcal{N}$ is a factor. The proof given in [68] is based on the deep results from [48] (see also $[66,50]$ ) concerning the structure of the commutator space $[I, J]$ spanned by all commutators $[T, S], T \in I, S \in J$ (here, $I$ and $J$ are ideals of compact operators from $\mathcal{L}(\mathcal{H})$ ). Note that every r.i. functional on the ideal $I$ vanishes on $[I, \mathcal{L}(\mathcal{H})]$. Many important results from $[48,66,50]$ admit an extension to the case of general semifinite von Neumann algebras and their ideals $[49,52]$

\section{Preliminaries on Dilation AND translation inVARiant States.}

A construction of Dixmier traces $\tau_{\omega}$ depends crucially on the choice of the "invariant mean" $\omega$. In this section we recall and review the most important classes of such means. We denote by $\ell_{\infty}=\ell_{\infty}(\mathbb{N})$ the Banach space of all bounded sequences of complex numbers. By a state on a unital $C^{*}$-algebra we mean a positive linear functional with value 1 on the unit of the algebra. We recall that a positive linear functional $\mathbb{L} \in \ell_{\infty}^{*}$ is called a Banach limit if $\mathbb{L}$ is translation invariant and $\mathbb{L}(\mathbf{1})=1$ (here, $\mathbf{1}=(1,1,1 \ldots)$ ). A Banach limit $\mathbb{L}$ satisfies in particular $\mathbb{L}(\xi)=0$ for all $\xi \in c_{0}$ (= all sequences from $\ell_{\infty}$ converging to zero). We denote the collection of all Banach limits on $\ell_{\infty}$ by $B L(\mathbb{N})$. Note that $\|\mathbb{L}\|=1$ for all $\mathbb{L} \in B L(\mathbb{N})$.

We recall that sequence $\xi=\left\{\xi_{n}\right\}_{n=1}^{\infty} \in \ell_{\infty}$ is said to be almost convergent to $\alpha \in \mathbb{R}$, denoted $F$ - $\lim _{n \rightarrow \infty} \xi_{n}=\alpha$ if and only if $\mathbb{L}(\xi)=\alpha$ for all $\mathbb{L} \in B L(\mathbb{N})$. The notion of an almost convergent sequence is due to G.G. Lorentz [75], who showed that the sequence $\left\{\xi_{n}\right\}_{n=1}^{\infty}$ is

almost convergent to $\alpha$ if and only if the equality $\lim _{p \rightarrow \infty} \frac{\xi_{n}+\xi_{n+1}+\cdots+\xi_{n+p-1}}{p}=\alpha$ holds uniformly for $n=1,2, \ldots$. We denote by $a c$ (respectively, $a c_{0}$ ) the set of all almost convergent (respectively, all almost convergent to 0 ) sequences from $\ell_{\infty}$. Clearly, $a c$ and $a c_{0}$ are closed subspaces in $\ell_{\infty}$. We define the shift operator $T: \ell_{\infty} \rightarrow \ell_{\infty}$, the Cesàro operator $H: \ell_{\infty} \rightarrow \ell_{\infty}$ and dilation operators $D_{n}: \ell_{\infty} \rightarrow \ell_{\infty}$ for $n \in \mathbb{N}$ by formulas

$$
\begin{gathered}
T\left(x_{1}, x_{2}, x_{3}, \ldots\right)=\left(x_{2}, x_{3}, x_{4}, \ldots\right) . \\
H\left(x_{1}, x_{2}, x_{3}, \ldots\right)=\left(x_{1}, \frac{x_{1}+x_{2}}{2}, \frac{x_{1}+x_{2}+x_{3}}{3}, \ldots\right), \\
D_{n}\left(x_{1}, x_{2}, x_{3}, \ldots\right)=(\underbrace{x_{1}, \ldots, x_{1}}_{n}, \underbrace{x_{2}, \ldots, x_{2}}_{n}, \ldots),
\end{gathered}
$$

for all $x=\left(x_{1}, x_{2}, x_{3}, \ldots\right) \in \ell_{\infty}$.

Each of the above operators is positive and leaves invariant the unit element 1 of the algebra $\ell_{\infty}$ and consequently is bounded with the norm equal to 1 . Moreover, $\left\{D_{n}\right\}_{n=1}^{\infty}$ is an abelian semigroup. The main tool in our construction of various classes of invariant means is the well known Markov-Kakutani fixed point theorem. 
Theorem 4.1 (Markov-Kakutani). Let $F$ be a locally convex Hausdorff space and let $K$ be a non-empty convex compact subset of $F$. Let $\mathcal{T}$ be an abelian semigroup of linear continuous operators on $F$ such that $S(K) \subseteq K$ for all $S \in \mathcal{T}$. Then there exists $x \in K$ such that $S x=x$ for all $S \in \mathcal{T}$.

It is easy to see that the set of all fixed points from Theorem 4.1 forms a convex compact subset of $K$.

We shall be applying the Markov-Kakutani theorem in the setting when $F=\left(\ell_{\infty}\right)^{*}$, $\left(L_{\infty}(\mathbb{R})\right)^{*},\left(L_{\infty}\left(\mathbb{R}_{+}^{*}\right)\right)^{*}$ equipped with the weak $*$-topology. For simplicity of exposition we present the proofs only for the first case.

Lemma $4.2([46,25])$. The following is true.

(i) $D_{n} T=T^{n} D_{n} \quad \forall n \geqslant 1$;

(ii) $H T x-T H x \in c_{0} \quad \forall x \in \ell_{\infty}$;

(iii) $H D_{n} x-D_{n} H x \in c_{0} \quad \forall x \in \ell_{\infty}$.

Proof. The proof of (i) is straightforward. For the proof of (ii) note that for all $x \in \ell_{\infty}$

$$
\left|(H T x)_{k}-(T H x)_{k}\right|=\left|\frac{1}{k+1} \frac{x_{2}+\cdots+x_{k+1}}{k}-\frac{1}{k+1} x_{1}\right| \leqslant \frac{2}{k+1}\|x\|_{\infty},
$$

which shows that $H T x-T H x \in c_{0}$.

We now indicate the proof of (iii). Let $n \geqslant 1$ and $x \in \ell_{\infty}$. For $1 \leqslant k \in \mathbb{N}$ there exist $l \geqslant 1$ and $1 \leqslant r \leqslant n$ such that $k=(l-1) n+r$. Hence

$$
\left(H D_{n} x\right)_{k}=\frac{1}{k} \sum_{i=1}^{k}\left(D_{n} x\right)_{i}=\frac{n}{k} \sum_{j=1}^{l-1} x_{j}+\frac{r}{k} x_{l}
$$

and

$$
\left(D_{n} H x\right)_{k}=(H x)_{l}=\frac{1}{l} \sum_{j=1}^{l} x_{j} .
$$

Noting that, $n l-k=n-r \leqslant n$ and $r l-k \leqslant n l-k \leqslant n$, it follows that

$$
\left|\left(H D_{n} x\right)_{k}-\left(D_{n} H x\right)_{k}\right|=\left|\frac{n l-k}{k l} \sum_{j=1}^{l-1} x_{j}+\frac{r l-k}{k l} x_{l}\right| \leqslant \frac{n}{k}\left(\frac{1}{l} \sum_{j=1}^{l-1}\left|x_{j}\right|\right)+\frac{n}{k}\left|x_{l}\right| \leqslant \frac{2 n}{k}\|x\|_{\infty} .
$$

This shows that $H D_{n} x-D_{n} H x \in \ell^{(1, \infty)} \subseteq c_{0}$.

Theorem 4.3. There exists a state $\tilde{\omega}$ on $\ell_{\infty}$ such that for all $n \geqslant 1$

$$
\tilde{\omega} \circ T=\tilde{\omega} \circ H=\tilde{\omega} \circ D_{n}=\tilde{\omega} \text {. }
$$

Proof. Let $K=\left\{0 \leqslant \varphi \in\left(\ell_{\infty}\right)^{*}: \varphi(\mathbf{1})=1, T^{*} \varphi=\varphi\right\}$. Since $K$ contains ordinary Banach limits it is not empty. It is clear that $K$ is convex and $*$-weakly compact. We claim that $D_{n}^{*}(K) \subseteq K$. Indeed, by Lemma $4.2(\mathrm{i})$ above we know that $T^{*} D_{n}^{*}=D_{n}^{*}\left(T^{*}\right)^{n}$, hence for $\varphi \in K$

$$
T^{*}\left(D_{n}^{*} \varphi\right)=D_{n}^{*}\left(T^{*}\right)^{n} \varphi=D_{n}^{*} \varphi
$$


which implies that $D_{n}^{*} \varphi \in K$. Therefore we may apply Theorem 4.1 to the set $K$ and the abelian semigroup $\left\{D_{j}^{*}\right\}_{j=1}^{\infty}$. Consequently the set

$$
K_{1}=\left\{0 \leqslant \varphi \in\left(\ell_{\infty}\right)^{*}: \varphi(\mathbf{1})=1, T^{*} \varphi=\varphi, D_{n}^{*} \varphi=\varphi, n \geqslant 1\right\}
$$

is non-empty and again it is clear that $K_{1}$ is convex and $*$-weakly compact.

We now show that $H^{*}\left(K_{1}\right) \subseteq K_{1}$. To this end, first observe that $\varphi(z)=0$ for all $z \in c_{0}$ and all $\varphi \in K_{1}$ (as $T^{*} \varphi=\varphi$ ). Given $\varphi \in K_{1}$ it follows from Lemma 4.2(iii) that

$$
\left(D_{n}^{*} H^{*} \varphi\right)(x)-\left(H^{*} D_{n}^{*} \varphi\right)(x)=\varphi\left(H D_{n} x-D_{n} H x\right)=0
$$

for all $x \in \ell_{\infty}$ and so

$$
D_{n}^{*}\left(H^{*} \varphi\right)=H^{*}\left(D_{n}^{*} \varphi\right)=H^{*} \varphi
$$

Similarly it follows from Lemma 4.2(ii) that $T^{*}\left(H^{*} \varphi\right)=H^{*} \varphi$ for all $\varphi \in K_{1}$. Consequently, $H^{*}\left(K_{1}\right) \subseteq K_{1}$. Applying Theorem 4.1 to the set $K_{1}$ and the semigroup $\left\{\left(H^{*}\right)^{n}\right\}_{n=0}^{\infty}$, we may conclude that there exists $\tilde{\omega} \in K_{1}$ such that $H^{*}(\tilde{\omega})=\tilde{\omega}$, by which the proof is complete.

We define the isomorphism $L: L_{\infty}(\mathbb{R}) \rightarrow L_{\infty}\left(\mathbb{R}_{+}^{*}\right)$ by $L(f)=f \circ \log$. Firstly, we define the Cesaro means (transforms) on $L_{\infty}(\mathbb{R})$ and $L_{\infty}\left(\mathbb{R}_{+}^{*}\right)$, respectively by:

$$
H(f)(u)=\frac{1}{u} \int_{0}^{u} f(v) d v \quad \text { for } \quad f \in L_{\infty}(\mathbb{R}), u \in \mathbb{R}
$$

and,

$$
M(g)(t)=\frac{1}{\log t} \int_{1}^{t} g(s) \frac{d s}{s} \quad \text { for } \quad g \in L_{\infty}\left(\mathbb{R}_{+}^{*}\right), t>0
$$

A brief calculation yields for $g \in L_{\infty}\left(\mathbb{R}_{+}^{*}\right)$,

$$
L H L^{-1}(g)(r)=\frac{1}{\log r} \int_{0}^{\log r} g\left(e^{u}\right) d u=\frac{1}{\log r} \int_{1}^{r} g(v) \frac{d v}{v}=M(g)(r),
$$

i.e $L$ intertwines the two means.

We shall now consider analogues of the operators $T, D_{n}$ and $H$ acting on $L_{\infty}(\mathbb{R})$ and $L_{\infty}\left(\mathbb{R}_{+}^{*}\right)$.

Definition 4.4. Let $T_{b}$ denote translation by $b \in \mathbb{R}, D_{a}$ denote dilation by $\frac{1}{a} \in \mathbb{R}_{+}^{*}$ and let $P^{a}$ denote exponentiation by $a \in \mathbb{R}_{+}^{*}$. That is,

$$
\begin{aligned}
T_{b}(f)(x) & =f(x+b) \quad \text { for } \quad f \in L_{\infty}(\mathbb{R}), \\
D_{a}(f)(x) & =f\left(\frac{x}{a}\right) \text { for } \quad f \in L_{\infty}(\mathbb{R}), \\
P^{a}(f)(x) & =f\left(x^{a}\right) \text { for } \quad f \in L_{\infty}\left(\mathbb{R}_{+}^{*}\right) .
\end{aligned}
$$

Some of the basic relations between these $L_{\infty}$ spaces and their self-maps are provided for easy access by the following proposition, whose proof is similar to Lemma 4.2.

Proposition $4.5([25]) . L_{\infty}(\mathbb{R})$ together with the self-maps, $D_{a}, T_{b}$, and $H(a>0, b \in \mathbb{R})$ is related to $L_{\infty}\left(\mathbb{R}_{+}^{*}\right)$ together with the self-maps, $P^{a}, D_{a}$, and $M(a>0)$ via the isomorphism

$$
L: L_{\infty}(\mathbb{R}) \rightarrow L_{\infty}\left(\mathbb{R}_{+}^{*}\right)
$$

and the following identities:

(1) $L D_{\frac{1}{a}} L^{-1}=P^{a}$ for $a>0$, 
(2) $L T_{b} L^{-1}=D_{(\exp (b))^{-1}}$ for $b \in \mathbb{R}$,

(3) $L H L^{-1}=M$,

(4) $D_{a} H=H D_{a}$ and $P^{a} M=M P^{a}$ for $a>0$,

(5) $\lim _{t \rightarrow \infty}\left(H T_{b}-T_{b} H\right) f(t)=0$ for $f \in L_{\infty}(\mathbb{R})$ and $b \in \mathbb{R}$,

(6) $\lim _{t \rightarrow \infty}\left(M D_{a}-D_{a} M\right) f(t)=0$ for $f \in L_{\infty}\left(\mathbb{R}_{+}^{*}\right)$ and $a>0$.

Proposition 4.6 ([25]). If a continuous functional $\tilde{\omega}$ on $L_{\infty}(\mathbb{R})$ is invariant under the Cesaro operator $H$, the shift operator $T_{a}$ or the dilation operator $D_{a}$ then $\tilde{\omega} \circ L^{-1}$ is a continuous functional on $L_{\infty}\left(\mathbb{R}_{+}^{*}\right)$ invariant under $M$, the dilation operator $D_{a}$ or $P^{a}$ respectively. Conversely, composition with $L$ converts an $M, D_{a}$ or $P^{a}$ invariant continuous functional on $L_{\infty}\left(\mathbb{R}_{+}^{*}\right)$ into an $H, T_{a}$ or $D_{a}$ invariant continuous functional on $L_{\infty}(\mathbb{R})$.

We denote by $C_{0}(\mathbb{R})$ (respectively, $C_{0}\left(\mathbb{R}_{+}^{*}\right)$ ) the continuous functions on $\mathbb{R}$ (respectively, $\mathbb{R}_{+}^{*}$ ) vanishing at infinity.

The proof of the following theorem is similar to that of Theorem 4.3.

Theorem $4.7([25])$. There exists a state $\tilde{\omega}$ on $L_{\infty}(\mathbb{R})$ satisfying the following conditions:

(1) $\tilde{\omega}\left(C_{0}(\mathbb{R})\right) \equiv 0$.

(2) If $f$ is real-valued in $L_{\infty}(\mathbb{R})$ then

$$
\text { ess } \liminf _{t \rightarrow \infty} f(t) \leqslant \tilde{\omega}(f) \leqslant e s s \limsup _{t \rightarrow \infty} f(t) .
$$

(3) If the essential support of $f$ is compact then $\tilde{\omega}(f)=0$.

(4) For all $a>0$ and $c \in \mathbb{R}$

$$
\tilde{\omega}=\tilde{\omega} \circ T_{c}=\tilde{\omega} \circ D_{a}=\tilde{\omega} \circ H
$$

Combining Theorem 4.7 and Proposition 4.6, we obtain

Corollary 4.8. There exists a state $\omega$ on $L_{\infty}\left(\mathbb{R}_{+}^{*}\right)$ satisfying the following conditions:

(1) $\omega\left(C_{0}\left(\mathbb{R}_{+}^{*}\right)\right) \equiv 0$.

(2) If $f$ is real-valued in $L_{\infty}\left(\mathbb{R}_{+}^{*}\right)$ then

$$
\text { ess } \liminf _{t \rightarrow \infty} f(t) \leqslant \omega(f) \leqslant e s s \limsup _{t \rightarrow \infty} f(t) .
$$

(3) If the essential support of $f$ is compact then $\omega(f)=0$.

(4) For all $a, c>0$

$$
\omega=\omega \circ D_{c}=\omega \circ P^{a}=\omega \circ M
$$

The results given in Theorem 4.7 and Corollary 4.8 allow one to exercise an alternative approach to the theory of Dixmier [41] and Connes-Dixmier traces [31]. Whereas Dixmier's original approach is based on the use of dilation invariant functionals, we replace the latter with Banach limits (= translation invariant functionals) and make use of the well-developed theory of almost convergent sequences. 
We introduce the following notation

$$
\begin{aligned}
& B L(\mathbb{R})=\left\{\text { the set of all states } \tilde{\omega} \text { on } L_{\infty}(\mathbb{R}) \text { satisfying conditions }(1)-(3) \text { of Theorem } 4.7\right. \\
& \text { such that } \left.\tilde{\omega} \circ T_{c}=\tilde{\omega} \text { for every } c \in \mathbb{R}\right\} \text {, } \\
& D\left(\mathbb{R}_{+}^{*}\right)=\left\{\text { the set of all states } \omega \text { on } L_{\infty}\left(\mathbb{R}_{+}^{*}\right) \text { satisfying conditions (1)-(3) of Corollary } 4.8\right. \\
& \text { such that } \left.\omega \circ D_{c}=\omega \text { for every } c \in \mathbb{R}_{+}^{*}\right\} \text {, } \\
& B L\left(\mathbb{R}_{+}\right)=\left\{\text {the set of all states } \tilde{\omega} \text { on } L_{\infty}\left(\mathbb{R}_{+}\right) \text {satisfying conditions (1)-(3) of Corollary } 4.8\right. \\
& \text { such that } \left.\tilde{\omega} \circ T_{c}=\tilde{\omega} \text { for every } c \in \mathbb{R}_{+}\right\} \text {. }
\end{aligned}
$$

The following simple remark plays an important role in the sequel. If $\omega \in D\left(\mathbb{R}_{+}^{*}\right)$, then $\mathbb{L}:=\omega \circ L$ belongs to $B L(\mathbb{R})$. If $\mathbb{L} \in B L\left(\mathbb{R}_{+}\right)$, then $\tilde{\omega}:=\mathbb{L} \circ L^{-1}$ belongs to $D\left(\mathbb{R}_{+}^{*}\right)$. Finally, note that the isomorphism $L: L_{\infty}(\mathbb{R}) \rightarrow L_{\infty}\left(\mathbb{R}_{+}^{*}\right)$ sends the space $C_{b}(\mathbb{R})$ of all bounded continuous functions on $\mathbb{R}$ onto the space $C_{b}\left(\mathbb{R}_{+}\right)$of all bounded continuous functions on $\mathbb{R}_{+}$. Thus, one can reformulate all the results from Propositions 4.5, 4.6, Theorem 4.7 and Corollary 4.8 for the spaces of continuous bounded functions on $\mathbb{R}$ and $\mathbb{R}_{+}$.

\section{Concrete Constructions of Singular symmetric functionals.}

5.1. Dixmier traces. If $\omega$ is a state on $\ell_{\infty}$ (respectively, on $\left.L_{\infty}(\mathbb{R}), L_{\infty}\left(\mathbb{R}_{+}^{*}\right)\right)$, then we shall frequently denote its value on the element $\left\{x_{i}\right\}_{i=1}^{\infty}$ (respectively, $f \in L_{\infty}(\mathbb{R}), L_{\infty}\left(\mathbb{R}_{+}^{*}\right)$ ) by $\omega-\lim _{i \rightarrow \infty} x_{i}$ (respectively, $\omega-\lim _{t \rightarrow \infty} f(t)$ ). Recall that Theorems 4.3, 4.7 and Corollary 4.8 guarantee the existence of translation and/or dilation invariant states on $\ell_{\infty}, L_{\infty}(\mathbb{R}), L_{\infty}\left(\mathbb{R}_{+}^{*}\right)$. For simplicity, we explain the construction of Dixmier traces for the ideal of compact operators $\left(\mathcal{L}^{(1, \infty)}(\mathcal{H}),\|\cdot\|_{(1, \infty)}\right)$ defined in Section 2.1.

Definition 5.1. Let $\omega$ be a $D_{2}$-invariant state on $\ell_{\infty}$. Dixmier trace of $T \in \mathcal{L}_{+}^{(1, \infty)}(\mathcal{H})$ is a number

$$
\tau_{\omega}(T):=\omega-\lim _{N \rightarrow \infty} \frac{1}{\log (1+N)} \sum_{n=1}^{N} \mu_{n}(T) .
$$

Remark. We have deliberately chosen $\omega$ to satisfy only the dilation invariance assumption in the proof below, even though Dixmier originally imposed on $\omega$ the assumption of dilation and translation invariance. We shall discuss differences below.

Proposition 5.2. $\tau_{\omega}(S+T)=\tau_{\omega}(S)+\tau_{\omega}(T) \quad \forall S, T \in \mathcal{L}_{+}^{(1, \infty)}(\mathcal{H})$.

Proof. Set, for brevity

$$
\sigma_{N}(X):=\sum_{n=1}^{N} \mu_{n}(X), \quad X \in \mathcal{L}^{(1, \infty)}(\mathcal{H})
$$

and note that

$$
\sigma_{N}(X)=\sup \{\operatorname{Tr}(X P): P=P(\mathcal{H}) \text { is a projection and } \operatorname{dim} P(\mathcal{H})=N\} .
$$


(see e.g. [56, 53]). For a given $\epsilon>0$, let projections $P_{1}$ and $P_{2}$ satisfy the conditions $\operatorname{dim} P_{1}(\mathcal{H})=\operatorname{dim} P_{2}(\mathcal{H})=N$ and $\operatorname{Tr}\left(S P_{1}\right)>\sigma_{N}(S)-\epsilon, \operatorname{Tr}\left(T P_{2}\right)>\sigma_{N}(T)-\epsilon$. Setting $P:=P_{1} \vee P_{2}$, we have

$$
\operatorname{Tr}((S+T) P)=\operatorname{Tr}(S P)+\operatorname{Tr}(T P) \geqslant \operatorname{Tr}\left(S P_{1}\right)+\operatorname{Tr}\left(T P_{2}\right)>\sigma_{N}(S)+\sigma_{N}(T)-2 \epsilon .
$$

Since $\operatorname{dim} P(\mathcal{H}) \leqslant 2 N$ and $\epsilon$ is an arbitrary positive number, we have

$$
\sigma_{2 N}(S+T) \geqslant \sigma_{N}(S)+\sigma_{N}(T), \quad N \geqslant 1 .
$$

Setting, for brevity,

$$
\alpha_{N}=\frac{1}{\log (1+N)} \sigma_{N}(S), \quad \beta_{N}=\frac{1}{\log (1+N)} \sigma_{N}(T), \quad \gamma_{N}=\frac{1}{\log (1+N)} \sigma_{N}(S+T) .
$$

we restate the above inequality as

$$
\frac{\log (2 N+1)}{\log (N+1)} \gamma_{2 N} \geqslant \alpha_{N}+\beta_{N}, \quad N \geqslant 1
$$

Assume, for a moment, that we know

$$
\omega-\lim _{N \rightarrow \infty} \gamma_{2 N}=\omega-\lim _{N \rightarrow \infty} \gamma_{N} .
$$

Noting that $\left\{\gamma_{2 N}\right\}_{N \geqslant 1} \in \ell_{\infty}$ and so $\frac{\log (1+2 N)}{\log (1+N)} \gamma_{2 N}-\gamma_{2 N} \rightarrow 0$, we infer from the above inequality that

$$
\tau_{\omega}(S+T)=\omega-\lim _{N \rightarrow \infty} \gamma_{N} \geqslant \omega-\lim _{N \rightarrow \infty} \alpha_{N}+\omega-\lim _{N \rightarrow \infty} \beta_{N}=\tau_{\omega}(S)+\tau_{\omega}(T) .
$$

Since the converse inequality $\tau_{\omega}(S+T) \leqslant \tau_{\omega}(S)+\tau_{\omega}(T)$ follows immediately from the wellknown inequality $\sigma_{N}(S+T) \leqslant \sigma_{N}(S)+\sigma_{N}(T)$ (see [56,53]), the proof is completed. It remains to explain equality (8).

Note that $D_{2}$-invariance of $\omega$ immediately implies that

$$
\omega\left(\left\{\gamma_{2 N}\right\}_{N=1}^{\infty}\right)=\omega\left(D_{2}\left\{\gamma_{2 N}\right\}_{N=1}^{\infty}\right)=\omega\left(\left\{\gamma_{2}, \gamma_{2}, \gamma_{4}, \gamma_{4}, \gamma_{6}, \gamma_{6}, \ldots\right\}\right)
$$

and therefore, in order to prove (8) it is sufficient to verify that

$$
D_{2}\left\{\gamma_{2 N}\right\}_{N=1}^{\infty}-\left\{\gamma_{N}\right\}_{N=1}^{\infty}=\left\{\gamma_{2}, \gamma_{2}, \gamma_{4}, \gamma_{4}, \gamma_{6}, \gamma_{6}, \ldots\right\}-\left\{\gamma_{1}, \gamma_{2}, \gamma_{3}, \gamma_{4}, \gamma_{5}, \ldots\right\} \in c_{0} \text {, }
$$

or equivalently, that $\gamma_{2 N}-\gamma_{2 N-1} \rightarrow 0$, as $N \rightarrow \infty$. For $N \geqslant 2$, we have

$$
\gamma_{2 N}-\gamma_{2 N-1}=\left(\frac{1}{\log 2 N}-\frac{1}{\log (2 N-1)}\right) \sigma_{2 N-1}(T+S)+\frac{1}{\log 2 N} \cdot \mu_{2 N}(T+S) .
$$

It is obvious that the second summand above tends to 0 as $N \rightarrow \infty$. Noting that the condition $T+S \in \mathcal{L}^{(1, \infty)}(\mathcal{H})$ guarantees $\sigma_{2 N-1}(T+S)=O(\log (2 N-1))$ and that $\frac{1}{\log 2 N}-\frac{1}{\log (2 N-1)}=$ $o\left(\frac{1}{\log (2 N-1)}\right)$, we see that the first summand also tends to 0 as $N \rightarrow \infty$.

Remark 5.3. Consider an isometric embedding $i: \ell_{\infty} \rightarrow L_{\infty}[0, \infty)$ given by $\left\{x_{j}\right\}_{j=1}^{\infty} \stackrel{i}{\mapsto}$ $\sum_{j=1}^{\infty} x_{j} \chi_{[j-1, j)}$, where $\chi_{[j-1, j)}$ is the characteristic function of the interval $[j-1, j)$. Observe that if $i\left(\left\{\gamma_{N}\right\}_{N \geqslant 1}\right)=f$, then $i\left(\left\{\gamma_{2 N}\right\}_{N \geqslant 1}\right)=f \circ D_{\frac{1}{2}}$. Therefore, the proof of (8) above would become trivial, if $\omega$ were a $D_{\frac{1}{2}}$-invariant state on $L_{\infty}[0, \infty)$.

Definition 5.4. Dixmier trace of a self-adjoint operator $T \in \mathcal{L}^{(1, \infty)}(\mathcal{H})$ is $\tau_{\omega}(T):=\tau_{\omega}\left(T_{+}\right)-$ $\tau_{\omega}\left(T_{-}\right)$and Dixmier trace of an arbitrary operator $T \in \mathcal{L}^{(1, \infty)}(\mathcal{H})$ is $\tau_{\omega}(T):=\tau_{\omega}(\operatorname{Re}(T))+$ $i \tau_{\omega}(\operatorname{Im}(T))$. 
Proposition 5.5. Dixmier trace $\tau_{\omega}$ is a (singular) symmetric functional on $\mathcal{L}^{(1, \infty)}(\mathcal{H})$, such that $\tau_{\omega}(S T)=\tau_{\omega}(T S)$ for every $T \in \mathcal{L}^{(1, \infty)}(\mathcal{H}), S \in B(\mathcal{H})$.

Proof. The inequality $\left|\tau_{\omega}(x)\right| \leqslant\|x\|_{(1, \infty)}, x \in \mathcal{L}^{(1, \infty)}(\mathcal{H})$, and further that $\tau_{\omega}$ is a symmetric functional follows immediately from Definition 5.1 and Corollary 4.8. Since every operator $S \in \mathcal{L}(\mathcal{H})$ is a linear combination of four unitary operators [84, VI.6], it is sufficient to prove the equality $\tau_{\omega}(U T)=\tau_{\omega}(T U)$ for a unitary $U$. Since every operator from $\mathcal{L}(\mathcal{H})$ is a linear combination of positive operators, it is sufficient to prove the last equality for positive $T$ 's. In this case, the latter equality follows immediately from the fact $\mu_{n}\left(U T U^{*}\right)=\mu_{n}(U T)=$ $\mu_{n}(T U)=\mu_{n}(T), \forall n \geqslant 1$.

Definitions 5.1 and 5.4 extend to Marcinkiewicz spaces $\mathcal{L}^{(1, \infty)}(\mathcal{N}, \tau)$ and further to Marcinkiewicz spaces $M(\psi)(\mathcal{N}, \tau)$, where $\psi \in \Omega$ satisfies condition (5). More precisely, fix an arbitrary state $\omega$ on $L_{\infty}\left(\mathbb{R}_{+}^{*}\right)$ satisfying conditions (1)-(3) of Corollary 4.8 which is $D_{\frac{1}{2}}$-invariant. Setting

$$
\tau_{\omega}(x):=\omega-\lim _{t \rightarrow \infty} a(x, t), \quad 0 \leqslant x \in M(\psi)(\mathcal{N}, \tau)
$$

and repeating a slightly modified argument (see the details in [47, p. 51]) from the proof of Propositions 5.2 and 5.5, we obtain an additive homogeneous functional on $M(\psi)(\mathcal{N}, \tau)_{+}$, which extends to a symmetric functional on $M(\psi)(\mathcal{N}, \tau)$ by linearity. In the sequel, we refer to any functional $\tau_{\omega}$ defined in $(9)$, where $\omega \in D\left(\mathbb{R}_{+}^{*}\right)$ as a Dixmier trace.

Finally, we note that the duality between the dilation invariant functionals on $L_{\infty}\left(\mathbb{R}_{+}^{*}\right)$ and translation invariant functionals on $L_{\infty}(\mathbb{R})$ allows an alternative definition of Dixmier traces. For simplicity, we consider this definition only for the space $\mathcal{L}^{(1, \infty)}(\mathcal{N}, \tau)$, where $(\mathcal{N}, \tau)$ is an arbitrary semifinite von Neumann algebra.

Let $\mathbb{L}$ (respectively, $\mathbb{L}$ ) belong to $B L\left(\mathbb{R}_{+}\right)$(respectively, $B L(\mathbb{N})$ ). We set

$$
\begin{aligned}
& F_{\mathbb{L}}(T):=\mathbb{L}-\lim _{t \rightarrow \infty} \frac{1}{\log \left(1+e^{t}\right)} \int_{0}^{e^{t}} \mu_{s}(T) d s, \\
& F_{\mathbb{L}}(T):=\mathbb{L}-\lim _{N \rightarrow \infty} \frac{1}{\log \left(1+e^{N}\right)} \sum_{n=1}^{\left[e^{N}\right]} \mu_{n}(T),
\end{aligned}
$$

where $\left[e^{N}\right]$ is the integral part of $e^{N}$.

Theorem 5.6. [ $[25,45,46,74]]$ For every semifinite von Neumann algebra $(\mathcal{N}, \tau)$ and arbitrary states $\mathbb{L} \in B L\left(\mathbb{R}_{+}\right)$and $\mathbb{L} \in B L(\mathbb{N})$, the functionals $F_{\mathbb{L}}$ and $F_{\mathbb{L}}$ are symmetric functionals on $\mathcal{L}^{(1, \infty)}(\mathcal{N}, \tau)$.

The following result shows that the class of Dixmier traces on $\mathcal{L}^{(1, \infty)}(\mathcal{N}, \tau)$ coincides with the sets of functionals $\left\{F_{\mathbb{L}}: \mathbb{L} \in B L(\mathbb{N})\right\}$ and $\left\{F_{\mathbb{L}}: \mathbb{L} \in B L\left(\mathbb{R}_{+}\right)\right\}$. The proof of the first equality below follows from the remarks at the end of the preceding section.

Theorem 5.7 ([74, Theorems 2.3,6.2]). For every semifinite von Neumann algebra $(\mathcal{N}, \tau)$, we have

$$
\left\{\tau_{\omega} \mid \omega \in D\left(\mathbb{R}_{+}^{*}\right)\right\}=\left\{F_{\mathbb{L}} \mid \mathbb{L} \in B L\left(\mathbb{R}_{+}\right)\right\}=\left\{F_{\mathbb{L}} \mid \mathbb{L} \in B L(\mathbb{N})\right\}
$$


The detailed study of the class of concave functions $\psi \in \Omega$ for which analogues of Theorems 5.6 and 5.7 hold for similarly defined classes of symmetric functionals on Marcinkiewicz spaces $M(\psi)(\mathcal{N}, \tau)$ is contained in $[45,46,74]$.

5.2. Connes-Dixmier traces. We have shown in the preceding subsection that with every state $\omega \in D\left(\mathbb{R}_{+}^{*}\right)$ (respectively, $\mathbb{L} \in B L\left(\mathbb{R}_{+}\right), B L(\mathbb{R}), \mathbb{L} \in B L(\mathbb{N})$ ) there exists an associated Dixmier trace $\tau_{\omega}$ (respectively, a symmetric functional $F_{\mathbb{L}}, F_{\mathbb{L}}$ ). It is possible to isolate various subsets in the sets of states $D\left(\mathbb{R}_{+}^{*}\right), B L\left(\mathbb{R}_{+}\right), B L(\mathbb{R}), B L(\mathbb{N})$ and relate with them corresponding subsets of traces. For example, let us consider the sets of all $H$-invariant (respectively, $M$-invariant) states on $L_{\infty}(\mathbb{R})\left(\right.$ or $\left.\ell_{\infty}\right)$ (respectively, $L_{\infty}\left(\mathbb{R}_{+}^{*}\right)$ ). It is easy to see that

$\left\{\omega: \quad \omega\right.$ is a $H$-invariant state on $L_{\infty}(\mathbb{R})\left(\right.$ resp. $\left.\left.\ell_{\infty}\right)\right\} \varsubsetneqq\{\omega: \omega \in B L(\mathbb{R}) \quad(\operatorname{resp} . B L(\mathbb{N}))\}$

and

$$
\left\{\omega: \quad \omega \text { is an } M \text {-invariant state on } L_{\infty}\left(\mathbb{R}_{+}^{*}\right)\right\} \varsubsetneqq\left\{\omega: \omega \in D\left(\mathbb{R}_{+}^{*}\right)\right\} .
$$

Indeed, suppose that $0 \leqslant \omega \in \ell_{\infty}^{*}$ is such that $\omega(\mathbf{1})=1$ and $\omega(H x)=\omega(x)$, for every $x \in \ell_{\infty}$. To prove $\omega(T x)=\omega(x), x \in \ell_{\infty}$, it is sufficient to show that $\omega(H T x)=\omega(H x), x \in \ell_{\infty}$. However, a straightforward calculation yields

$$
(H T x)_{N}-(H x)_{N}=\frac{x_{2}+\ldots+x_{N+1}}{N}-\frac{x_{1}+\ldots+x_{N}}{N}=\frac{x_{N+1}-x_{1}}{N} \rightarrow 0, \text { as } \quad N \rightarrow \infty .
$$

Similarly, it can be shown that for every $x \in L_{\infty}(\mathbb{R})$ and $b \in \mathbb{R}$ we have

$$
\lim _{t \rightarrow \infty}\left(H T_{b} x\right)(t)-(H x)(t)=0
$$

which establishes (10) and the inclusion (11) follows from (10) via Corollary 4.8. Alain Connes in [31] suggested to work with the set of states on $L_{\infty}\left(\mathbb{R}_{+}^{*}\right)$, which is larger then the set on the left hand side of (11). Namely, let us consider the following class of states on $L_{\infty}\left(\mathbb{R}_{+}^{*}\right)$

$$
C D\left(\mathbb{R}_{+}^{*}\right):=\left\{\tilde{\omega}=\gamma \circ M: \gamma \text { is an arbitrary singular state on } C_{b}[0, \infty)\right\} .
$$

It is still easy to verify that $C D\left(\mathbb{R}_{+}^{*}\right) \varsubsetneqq D\left(\mathbb{R}_{+}^{*}\right)$, and then infer the proper inclusion $C B L\left(\mathbb{R}_{+}\right) \varsubsetneqq B L\left(\mathbb{R}_{+}\right)$, where

$$
C B L\left(\mathbb{R}_{+}\right):=\left\{\mathbb{L}=\gamma \circ H: \gamma \text { is an arbitrary singular state on } C_{b}\left(\mathbb{R}_{+}\right)\right\},
$$

from Proposition 4.5. We refer to the subclass of Dixmier traces

$$
\left\{\tau_{\omega}: \omega \in C D\left(\mathbb{R}_{+}^{*}\right)\right\}
$$

as the class of Connes-Dixmier traces. The following theorem shows that an analogue of Theorem 5.7 also holds for the class of Connes-Dixmier traces.

Theorem 5.8 ([74, Theorems 5.6, 6.2]). For every semi-finite von Neumann algebra $(\mathcal{N}, \tau)$, we have

$$
\left\{\tau_{\omega}: \omega \in C D\left(\mathbb{R}_{+}^{*}\right)\right\}=\left\{F_{\mathbb{L}}: \quad \mathbb{L} \in C B L\left(\mathbb{R}_{+}\right)\right\}
$$

We complete this subsection with the remark that there is another natural subclass of Dixmier traces which is associated with the subset of states on $L_{\infty}\left(\mathbb{R}_{+}^{*}\right)$ appearing in Corollary 4.8

$\left\{\omega \in L_{\infty}\left(\mathbb{R}_{+}^{*}\right)^{*}: \quad \omega\right.$ is an $M$-invariant and $P^{a}$-invariant state on $\left.L_{\infty}\left(\mathbb{R}_{+}^{*}\right), a>0\right\}$, 
or equivalently, with the set

$$
\left\{\mathbb{L} \in L_{\infty}(\mathbb{R})^{*}: \mathbb{L} \text { is an } H \text {-invariant and } D_{a} \text {-invariant state on } L_{\infty}(\mathbb{R}), a>0\right\} .
$$

The class of Dixmier traces associated with the latter set is further referred to as the class of maximally invariant Dixmier traces. Clearly, the latter class is contained in the class of Connes-Dixmier traces. Maximally invariant Dixmier traces are used in Sections 10 and 11.

5.3. Rearrangement invariant functionals and singular traces. The class of Dixmier traces (and its subclasses) studied in the preceding subsections is a special subclass of the general class of (singular) symmetric functionals on the (fully symmetric) operator space $\mathcal{L}^{(1, \infty)}(\mathcal{N}, \tau)$ (or, on operator ideal $\mathcal{L}^{(1, \infty)}(\mathcal{H})$ ). The latter class is, in its turn, a subclass of the class of r.i. functionals on $\mathcal{L}^{(1, \infty)}(\mathcal{N}, \tau)$. In the case of the operator ideal $\mathcal{L}^{(1, \infty)}(\mathcal{H})$, the latter class may be viewed as the set of all (singular) traces on $\mathcal{L}(\mathcal{H})$, which take finite values on the elements from $\mathcal{L}^{(1, \infty)}(\mathcal{H})$. A literature devoted to general singular traces on $\mathcal{L}(\mathcal{H})$ is tremendous. We limit our list of papers from this area to the following articles $[58,59,2,3,4,26,92]$. In many cases, the theory of singular traces runs in parallel with the theory of symmetric functionals. For instance, results of $[2,92]$ (respectively, [58]) concerning necessary and sufficient conditions on a positive compact operator $T$ (respectively, positive $\tau$-measurable operators $\left.T \in L_{1}(\mathcal{N}, \tau)+\mathcal{N}\right)$ for the existence of a singular trace which takes a finite non-zero value on $T$ are in fact very close to the result of Theorem 2.2. It should be noted that the results [58] are stated for general $\tau$-measurable operators and not just for the operators from $L_{1}(\mathcal{N}, \tau)+\mathcal{N}$, however not all the results there treating this general case are supplied with a reliable proof (e.g. [58, Proposition 3.3]).

Finally, we point out at the important connections between the theory of general singular traces and the study of the structure of commutator spaces for operators acting on a Hilbert space (see the comments made at the end Section 3). The latter study is related to cyclic homology and algebraic $K$-theory of operator ideals and is beyond the scope of the present survey. For details, we recommend [48, 66, 49, 50, 52].

\section{Class of measurable elements.}

In this section, we briefly review the notion of measurable operators introduced by A.Connes [31].

Definition 6.1. $T \in \mathcal{L}^{(1, \infty)}(\mathcal{H})$ is called (Dixmier)-measurable if $\tau_{\omega}(T)$ does not depend on the choice of $\omega \in D\left(\mathbb{R}_{+}^{*}\right)$.

Definition $6.2([31]) . T \in \mathcal{L}^{(1, \infty)}(\mathcal{H})$ is called (Connes-Dixmier)-measurable if $\tau_{\omega}(T)$ does not depend on the choice of $\omega \in C D\left(\mathbb{R}_{+}^{*}\right)$.

Remark 6.3. (i). It is obvious that the sets of measurable operators defined above are linear spaces, which are, in fact, closed subspaces of $\mathcal{L}^{(1, \infty)}(\mathcal{H})$. However, these subspaces are not order ideals, in other words, the fact that a self-adjoint operator $A$ is measurable does not necessarily imply that $A_{+}$and $A_{-}$are measurable operators. Example. Take a positive non-measurable diagonal operator $A=\operatorname{diag}\left\{a_{1}, a_{2}, a_{3}, \ldots\right\}$ from $\mathcal{L}^{(1, \infty)}(\mathcal{H})$. Define a diagonal operator $B$ by $B=\operatorname{diag}\left\{a_{1},-a_{1}, a_{2},-a_{2}, \ldots\right\}$. Evidently, $B$ is measurable, moreover $\tau_{\omega}(B)=0$ for all $\omega$. However, the positive and negative parts of $B$ are not measurable. 
(ii). Definitions of Dixmier and Connes-Dixmier measurable operators naturally extend [74] to Marcinkiewicz spaces $\mathcal{L}^{(1, \infty)}(\mathcal{N}, \tau)$, where $(\mathcal{N}, \tau)$ is an arbitrary semifinite von Neumann algebra, and further, to operator Marcinkiewicz spaces $M(\psi)(\mathcal{N}, \tau)$, for all $\psi$ satisfying condition (3).

It is obvious, that every Dixmier-measurable operator is also Connes-Dixmier measurable. Our objective in the present section is to describe the classes of positive Connes-Dixmier measurable operators and positive Dixmier-measurable operators and to show that these two classes actually coincide. To this end, we will need two auxiliary results.

Theorem 6.4. [63, section 6.8] Let $b(t)$ be a positive piecewise differentiable function such that $t b^{\prime}(t)>-H$ for some $H>0$ and all $t>C$, where $C$ is a constant. Then

$$
\lim _{t \rightarrow \infty} \frac{1}{t} \int_{0}^{t} b(s) d s=A \text { for some } \quad A \geqslant 0 \quad \text { if and only if } \quad \lim _{t \rightarrow \infty} b(t)=A .
$$

Imitating the Lorentz definition of almost convergent sequences, a positive function $f \in$ $C_{b}[0, \infty)$ is said to be almost convergent if all states from $B L\left(\mathbb{R}_{+}\right)$take the same value on this function.

Theorem 6.5. [74, Theorem 3.3] If a function $f \in C_{b}[0, \infty)$ is almost convergent to a number $A$ then, the following limit

$$
\lim _{t \rightarrow \infty} \frac{1}{t} \int_{0}^{t} f(s) d s
$$

exists and is equal to $A$.

Proof. Suppose the result is false. Then there exists a constant $c \neq A$ such that $(H f)\left(t_{n}\right) \rightarrow$ $c$ for some sequence $t_{n} \uparrow \infty$. Take the unit ball $B$ of $C_{b}([0, \infty))^{*}$ and consider the sequence of functionals $\sigma_{t_{n}}(x)=x\left(t_{n}\right), n \geqslant 1$ from $B$. Since $B$ is weak*-compact, this sequence has a limit point $V \in B$. It is easy to see that $V \geqslant 0, V(1)=1, V(p)=\lim _{n \rightarrow \infty} p\left(t_{n}\right)=0$ for every $p \in C_{0}[0, \infty)$ and also that $V(H(f))=\lim _{n \rightarrow \infty} H(f)\left(t_{n}\right)=c$. Define the functional $L$ on $L_{\infty}\left(\mathbb{R}_{+}\right)$by setting $L(x):=V(H(x))$. It is easy to verify that $L$ is a state from $B L\left(\mathbb{R}_{+}\right)$ and that $V(f)=c \neq A$. Thus, the supposition that the result does not hold is false.

The following theorem is the main result of this section.

Theorem 6.6. [74] A positive operator $T$ from $\mathcal{L}^{(1, \infty)}(\mathcal{N}, \tau)$ is Dixmier-measurable if and only if the limit

$$
\lim _{t \rightarrow \infty} \frac{1}{\log (1+t)} \int_{0}^{t} \mu_{s}(T) d s
$$

exists.

Proof. The "if" part of the assertion is trivial. Now, fix an operator $T \in \mathcal{L}_{+}^{(1, \infty)}(\mathcal{N}, \tau)$ such that for $g(t):=\frac{1}{\log (1+t)} \int_{0}^{t} \mu_{s}(T) d s$, we have $\tau_{\omega}(T)=\omega$ - $\lim _{t \rightarrow \infty} g(t)=A \geqslant 0$ for every $\omega \in C D\left(\mathbb{R}_{+}^{*}\right)$. It follows from the remarks made in Section 5.2 that for all $\mathbb{L} \in B L\left(\mathbb{R}_{+}\right)$, we have $\operatorname{Tr}_{\mathbb{L}}(T):=\mathbb{L}-\lim _{\lambda \rightarrow \infty} g\left(e^{\lambda}\right)=A$, and therefore, by Theorem 6.5 , we obtain

$$
\lim _{u \rightarrow \infty} \frac{1}{u} \int_{0}^{u}\left(\frac{1}{\log \left(1+e^{\lambda}\right)} \int_{0}^{e^{\lambda}} \mu_{s}(T) d s\right) d \lambda=A .
$$


Setting,

we have

$$
b(\lambda):=\frac{1}{\log \left(1+e^{\lambda}\right)} \int_{0}^{e^{\lambda}} \mu_{s}(T) d s, \quad \lambda>0
$$

$$
\begin{aligned}
& \lambda b^{\prime}(\lambda) \geqslant \lambda \frac{d}{d \lambda}\left(\frac{1}{\log \left(1+e^{\lambda}\right)}\right) \int_{0}^{e^{\lambda}} \mu_{s}(T) d s=-\frac{\lambda e^{\lambda}}{\left(1+e^{\lambda}\right) \log ^{2}\left(1+e^{\lambda}\right)} \int_{0}^{e^{\lambda}} \mu_{s}(T) d s \\
& \geqslant-\frac{\lambda}{\log \left(1+e^{\lambda}\right)} \cdot \frac{1}{\log \left(1+e^{\lambda}\right)} \int_{0}^{e^{\lambda}} \mu_{s}(T) d s \geqslant-\|T\|_{(1, \infty)} .
\end{aligned}
$$

Applying Theorem 6.4, we now infer that $\lim _{\lambda \rightarrow \infty} b(\lambda)=A$, and therefore $\lim _{t \rightarrow \infty} g(t)=A$.

We shall now show that a similar argument as in the proof above yields a stronger result.

Theorem 6.7 ([74]). A positive operator $T$ from $\mathcal{L}^{(1, \infty)}(\mathcal{N}, \tau)$ is Connes-Dixmier-measurable if and only if the limit

$$
\lim _{t \rightarrow \infty} \frac{1}{\log (1+t)} \int_{0}^{t} \mu_{s}(T) d s
$$

exists.

Proof. We need only to show the "only if" part. We shall use the notations $g(\cdot)$ and $b(\cdot)$ introduced in the proof of Theorem 6.6. Suppose that $T \in \mathcal{L}_{+}^{(1, \infty)}(\mathcal{N}, \tau)$ satisfies the equality $A=\gamma \circ M(g)=\gamma \circ L H L^{-1}(g)$ for every state $\gamma \in C_{b}^{*}[0, \infty)$ vanishing on $C_{0}(0, \infty)$.

Note that if $\gamma$ is dilation invariant state, then $\gamma \circ L$ is a translation invariant state. This remark (and the fact that $L: L_{\infty}(\mathbb{R}) \rightarrow L_{\infty}\left(\mathbb{R}_{+}^{*}\right)$ is an isomorphism) show that $H L^{-1}(g)=H b$ is almost convergent. Applying Theorem 6.5 to the function $H b$, we see that the limit $\lim _{t \rightarrow \infty} H H(b)(t)$ exists. Assume, for a moment, that we have already verified the assumption of Theorem 6.4 for the function $H b$. Then, we infer from that theorem that the limit $\lim _{t \rightarrow \infty} H(b)(t)$ also exists, and repeating the application of the same theorem (as in the proof of Theorem 6.6), we conclude that there exists the $\operatorname{limit}_{\lim _{t \rightarrow \infty}} b(t)$, and hence the limit $\lim _{\lambda \rightarrow \infty} g(\lambda)$.

It remains to verify the assumption of Theorem 6.4 for $H b$. We have for all $\lambda \geqslant 1$

$$
\lambda(H b)^{\prime}(\lambda)=\frac{\lambda b(\lambda)-\int_{0}^{\lambda} b(s) d s}{\lambda} \geqslant-\|b\|_{\infty} \geqslant-\|T\|_{(1, \infty)} .
$$

Corollary 6.8. The set of all positive Dixmier measurable operators and the set of all positive Connes-Dixmier measurable operators coincide.

The following questions are open:

(i). Do the spaces of Dixmier measurable and Connes-Dixmier measurable operators coincide? 
(ii). What is the description of the set of all (positive) operators, which are measurable with respect to the set of all maximally invariant Dixmier traces?

(iii). What is the description of the set of all (positive) operators measurable with respect to the set of all symmetric functionals?

One might be tempted to pose question (iii) in greater generality and ask whether the concept of operators measurable with respect to the set of all r.i. functionals can be sensibly formulated. There is an example due to [68] (see the end of Section 2 above) which answers this in the negative.

For results extending Theorems 6.6 and 6.7 to Marcinkiewicz spaces $M(\psi)(\mathcal{N}, \tau)$, with $\psi \in \Omega$ satisfying condition (4), we refer the reader to [74].

\section{Norming Properties of Dixmier and Connes-Dixmier functionals}

A reader may have an impression that Dixmier and Connes-Dixmier traces form a very "thin" subset of the unit sphere of the dual space. Such an impression is wrong as established by Theorems 7.3 and 7.4 below. We shall need the following theorem of Sucheston.

Theorem $7.1([90])$. For $x \in \ell_{\infty}$

$$
\sup _{\mathbb{L} \in B L(\mathbb{N})} \mathbb{L}(x)=\lim _{n \rightarrow \infty}\left(\sup _{m} \frac{1}{n} \sum_{j=1}^{n} x_{m+j}\right) .
$$

The following proposition easily follows from its "commutative" counterpart, which, in its turn, can be obtained from [69].

Proposition 7.2. Let $T \in \mathcal{L}^{(1, \infty)}(\mathcal{N}, \tau)$. The distance from $T$ to the subspace $\mathcal{L}_{0}^{(1, \infty)}(\mathcal{N}, \tau)$ in the norm $\|\cdot\|_{(1, \infty)}$ is equal to

$$
\rho(T):=\limsup _{t \rightarrow \infty} \frac{1}{\log (1+t)} \int_{0}^{t} \mu_{s}(T) d s .
$$

Recall, that in the special case $\mathcal{L}^{(1, \infty)}(\mathcal{N}, \tau)=\mathcal{L}^{(1, \infty)}(\mathcal{H})$, the space $\mathcal{L}_{0}^{1, \infty}(\mathcal{H})$ is the closed linear span in $\mathcal{L}^{(1, \infty)}(\mathcal{H})$ of the set of all finite-dimensional operators.

Theorem 7.3. [74] Let $T \in \mathcal{L}^{(1, \infty)}(\mathcal{N}, \tau)$. The distance from $T$ to the subspace $\mathcal{L}_{0}^{(1, \infty)}(\mathcal{N}, \tau)$ in the norm $\|\cdot\|_{(1, \infty)}$ is equal to $\sup \left\{\tau_{\omega}(|T|): \omega \in D\left(\mathbb{R}_{+}^{*}\right)\right\}$.

Proof. It is sufficient to consider the case $T \geqslant 0$. We note first that

$$
\sup \left\{\tau_{\omega}(T): \omega \in D\left(\mathbb{R}_{+}^{*}\right)\right\}=\sup \left\{\operatorname{Tr}_{\mathbb{L}}(T): \mathbb{L} \in B L\left(\mathbb{R}_{+}\right)\right\}=\sup \left\{\operatorname{Tr}_{\mathbb{L}}(T): \mathbb{L} \in B L(\mathbb{N})\right\} .
$$

It is clear that

$$
q(T):=\sup _{\mathbb{L} \in B L(\mathbb{N})} \operatorname{Tr}_{\mathbb{L}}(T) \leqslant \limsup _{t \rightarrow \infty} \frac{1}{\log (1+t)} \int_{0}^{t} \mu_{s}(T) d s(=\rho(T)) .
$$


We have to prove the reverse inequality $q(T) \geqslant \rho(T)$. By Sucheston's theorem 7.1, it is enough to prove that $\forall \epsilon>0 \exists N \in \mathbb{N} \forall n \geqslant N \exists m \in \mathbb{N}$ such that

$$
\frac{1}{n} \sum_{j=1}^{n} \frac{1}{m+j} \int_{0}^{e^{m+j}} \mu_{s}(T) d s \geqslant \rho(T)-\epsilon
$$

For this purpose, it is enough to put $N=1$ and to take $m$ such that $\frac{m}{m+n}>\frac{\rho(T)-\epsilon}{\rho(T)-\epsilon / 2}$ and

$$
\frac{1}{\log \left(1+e^{m}\right)} \int_{0}^{e^{m}} \mu_{s}(T) d s>\rho(T)-\epsilon / 2
$$

Then

$$
\frac{1}{n} \sum_{j=1}^{n} \frac{1}{m+j} \int_{0}^{e^{m+j}} \mu_{s}(T) d s \geqslant \frac{1}{n} \sum_{j=1}^{n} \frac{1}{m+n} \int_{0}^{e^{m}} \mu_{s}(T) d s=\frac{m}{m+n} \cdot \frac{1}{m} \int_{0}^{e^{m}} \mu_{s}(T) d s>\rho(T)-\epsilon .
$$

To verify that selection of $m$ satisfying (12) is feasible, first we locate a sequence $1 \leqslant t_{1}<$ $t_{2} \ldots \uparrow \infty$, such that

$$
\lim _{k \rightarrow \infty} \frac{1}{\log \left(1+t_{k}\right)} \int_{0}^{t_{k}} \mu_{s}(T) d s>\rho(T)-\epsilon / 4, \quad k \geqslant 1,
$$

(this may be done due to Proposition 7.2). For every $k$, we define $m_{k} \in \mathbb{N}$, so that $e^{m_{k}-1} \leqslant$ $t_{k} \leqslant e^{m_{k}}$. Then

$$
\begin{aligned}
\frac{1}{\log \left(1+t_{k}\right)} \int_{0}^{t_{k}} \mu_{s}(T) d s & \leqslant \frac{1}{\log \left(1+e^{m_{k}-1}\right)} \int_{0}^{e^{m_{k}}} \mu_{s}(T) d s \\
& =\frac{\log \left(1+e^{m_{k}}\right)}{\log \left(1+e^{m_{k}-1}\right)} \cdot \frac{1}{\log \left(1+e^{m_{k}}\right)} \int_{0}^{e^{m_{k}}} \mu_{s}(T) d s .
\end{aligned}
$$

Since $\frac{\log \left(1+e^{m} k\right)}{\log \left(1+e^{m} k^{-1}\right)} \rightarrow 1$, we see that (12) follows from (13).

A natural question is whether the norming property remains true for the class of ConnesDixmier traces, as answered below.

Theorem 7.4. [74] Let $T \in \mathcal{L}^{(1, \infty)}(\mathcal{N}, \tau)$. The distance from $T$ to the subspace $\mathcal{L}_{0}^{(1, \infty)}(\mathcal{N}, \tau)$ in the norm $\|\cdot\|_{(1, \infty)}$ is equivalent to $\sup \tau_{\omega}(T)$, where the supremum is taken over all singular states $\omega=\gamma \circ M$, where $\gamma$ is a singular state on $C_{b}[0, \infty)$.

As in the preceding section, the results given in Theorems 7.3 and 7.4 admit an extension to Marcinkiewicz spaces $M(\psi)(\mathcal{N}, \tau)$ with $\psi \in \Omega$ satisfying condition (4).

We finish this section with the comment that it is not clear yet, whether the difference in the results of Theorems 7.3 and 7.4 signify that the set of all Dixmier traces is different from the set of all Connes-Dixmier traces. 


\section{Fredholm modules ANd SPECtral triples}

8.1. Notation and definitions. Let $\mathcal{N}$ be a semifinite von Neumann algebra on a separable Hilbert space $\mathcal{H}$ and let $L_{p}(\mathcal{N}, \tau)$ be a non-commutative $L_{p}$-space associated with $(\mathcal{N}, \tau)$, where $\tau$ is a faithful, normal semifinite trace on $\mathcal{N}$. Let $\mathcal{A}$ be a unital Banach $*$-algebra which is represented in $\mathcal{N}$ via a continuous $*$-homomorphism $\pi$ which, without loss of generality, we may assume to be faithful. Where no confusion arises we suppress $\pi$ in the notation. The fundamental objects of our analysis are explained in the following definition.

Let $\mathcal{K}_{\mathcal{N}}$ be the $\tau$-compact operators in $\mathcal{N}$ (that is the norm closed ideal generated by the projections $E \in \mathcal{N}$ with $\tau(E)<\infty)$.

Definition 8.1. (i) A semifinite odd spectral triple $(\mathcal{A}, \mathcal{H}, \mathcal{D})$ is given by a Hilbert space $\mathcal{H}$, a $*$-algebra $\mathcal{A} \subset \mathcal{N}$ where $\mathcal{N}$ is a semifinite von Neumann algebra acting on $\mathcal{H}$, and a densely defined unbounded self-adjoint operator $\mathcal{D}$ affiliated to $\mathcal{N}$ such that

1) $[\mathcal{D}, a]$ is densely defined and extends to a bounded operator for all $a \in \mathcal{A}$

2) $(\lambda-\mathcal{D})^{-1} \in \mathcal{K}_{\mathcal{N}}$ for all $\lambda \notin \mathbb{R}$

(ii) We say that $(\mathcal{A}, \mathcal{H}, \mathcal{D})$ is even if in addition there is a $\mathbf{Z}_{2}$-grading such that $\mathcal{A}$ is even and $\mathcal{D}$ is odd. That is an operator $\Gamma$ such that $\Gamma=\Gamma^{*}, \Gamma^{2}=1, \Gamma a=a \Gamma$ for all $a \in \mathcal{A}$ and $\mathcal{D} \Gamma+\Gamma \mathcal{D}=0$.

(iii) If $\mathcal{I}$ is a symmetrically normed ideal in $\mathcal{K}_{\mathcal{N}}$ then we say that the spectral triple $(\mathcal{A}, \mathcal{N}, \mathcal{D})$ is $\mathcal{I}$-summable if $\left(1+\mathcal{D}^{2}\right)^{-1 / 2} \in \mathcal{I}$.

Remark 8.2. In [10] the terminology for the concept we have just introduced is 'von Neumann spectral triple'. The two most important special cases of this definition are when

a) $\mathcal{I}$ is the ideal $L^{p}$ in which case we say $(\mathcal{A}, \mathcal{N}, \mathcal{D})$ is $p$-summable and

b) the case where the Dixmier trace was first evident namely $\mathcal{I}=\mathcal{L}^{p, \infty}$. In this case, we say that $(\mathcal{A}, \mathcal{N}, \mathcal{D})$ is $(p, \infty)$-summable.

There is a third case which is not relevant for the discussion here and that is the notion of theta summability $[29,30,31]$.

Note. In this paper, for simplicity of exposition, we will deal only with unital algebras $\mathcal{A} \subset \mathcal{N}$ where the identity of $\mathcal{A}$ is that of $\mathcal{N}$. We have adopted a notational convention correlated to the context in which we are working. A calligraphic $\mathcal{D}$ will always denote an unbounded self-adjoint operator forming part of a semifinite spectral triple $(\mathcal{A}, \mathcal{H}, \mathcal{D})$. A roman $D$ will denote a self-adjoint operator on a Hilbert space, usually with some side conditions.

In the original paper on noncommutative geometry by Alan Connes [28] the notion of spectral triple was introduced as an 'unbounded Fredholm module' (see also [31]). The study of semifinite spectral triples was initiated in [19] in the context of spectral flow [5, 6, 80] and carried further in [10] with a focus on applications to foliations. In [28] the notion of Fredholm module was introduced based on the idea of a Kasparov module. This may be generalised to the semifinite case as follows.

Definition 8.3. ([19], [91]) A bounded $p$-summable pre-Breuer-Fredholm module for $\mathcal{A}$, is a pair $\left(\mathcal{N}, F_{0}\right)$ where $F_{0}$ is a bounded self-adjoint operator in $\mathcal{N}$ satisfying: 
(1) $\left|\mathbf{1}-F_{0}^{2}\right|^{1 / 2}$ belongs to $L_{p}(\mathcal{N}, \tau)$; and

(2) $\left.\mathcal{A}_{p}:=\left\{a \in \mathcal{A} \mid\left[F_{0}, a\right] \in L_{p}(\mathcal{N}, \tau)\right\}\right)$ is a dense $*$-subalgebra of $\mathcal{A}$.

When $F_{0}^{2}=\mathbf{1}$ we drop the prefix 'pre-'.

In the special case when $\mathcal{N}=\mathcal{L}(\mathcal{H})$ and $\tau$ is the standard trace Tr, we omit "Breuer" from the definition. In this case, the non-commutative $L_{p}$-space coincides with the Schatten-von Neumann ideal $\mathcal{C}_{p}$ of compact operators and the definition originates from Definition 3 from [31] p.290.

8.2. Bounded versus unbounded. The relationship between the bounded and unbounded pictures is worth some comment. There are two approaches to this question, that in [87] (and explained in more detail in [57]) and a more general approach via perturbation theory in [24]. In the case of even spectral triples this matter was settled by Connes (see [28] I.6). Let $D$ and $D_{0}$ be unbounded self adjoint operators on $\mathcal{H}$ differing by a bounded operator in $\mathcal{N}$. We study directly the map $\varphi$ defined by $\varphi(D)=D\left(1+D^{2}\right)^{-1 / 2}$ and the difference $\varphi(D)-\varphi\left(D_{0}\right)$. In [24] a number of results were established, the one most relevant to this survey being:

Theorem 8.4. With the assumptions above on $D$ and $D_{0}$ and with $D-D_{0} \in \mathcal{N}$ and $1<p<\infty$ we have

$$
\left\|\varphi(D)-\varphi\left(D_{0}\right)\right\|_{L_{p}(\mathcal{N}, \tau)} \leqslant Z_{p} \max \left\{\left\|D-D_{0}\right\|^{1 / 2},\left\|D-D_{0}\right\|\right\} \cdot\left\|\left(1+D^{2}\right)^{-1 / 2}\right\|_{L_{p}(\mathcal{N}, \tau)} .
$$

for some positive constant $Z_{p}$ which depends on $p$ only.

From this theorem (cf [87]) one obtains the

Corollary 8.5. If $1<p<\infty$ and $\left(\mathcal{A}, \mathcal{N}, \mathcal{D}_{0}\right)$ is an odd semifinite $p$-summable spectral triple for the Banach $*$-algebra then $\left(\mathcal{N}, \operatorname{sign}\left(\mathcal{D}_{0}\right)\right)$ is an odd bounded $p$-summable Breuer-Fredholm module for $\mathcal{A}$.

\subsection{More on Semifinite Spectral Triples.}

Definition 8.6. A semifinite spectral triple $(\mathcal{A}, \mathcal{H}, \mathcal{D})$ is $Q C^{k}$ for $k \geqslant 1$ ( $Q$ for quantum) if for all $a \in \mathcal{A}$ the operators $a$ and $[\mathcal{D}, a]$ are in the domain of $\delta^{k}$, where $\delta(T)=[|\mathcal{D}|, T]$ is the partial derivation on $\mathcal{N}$ defined by $|\mathcal{D}|$.

Notes. (i) The notation is meant to be analogous to the classical case, but we introduce the $Q$ so that there is no confusion between quantum differentiability of $a \in \mathcal{A}$ and classical differentiability of functions.

(ii) By a partial derivation we mean that $\delta$ is defined on some subalgebra of $\mathcal{N}$ which need not be (weakly) dense in $\mathcal{N}$. More precisely, $\operatorname{dom} \delta=\{T \in \mathcal{N}: \delta(T)$ is bounded $\}$.

Observation If $T \in \mathcal{N}$, one can show that $[|\mathcal{D}|, T]$ is bounded if and only if $\left[\left(1+\mathcal{D}^{2}\right)^{1 / 2}, T\right]$ is bounded, by using the functional calculus to show that $|\mathcal{D}|-\left(1+\mathcal{D}^{2}\right)^{1 / 2}$ extends to a bounded operator in $\mathcal{N}$. In fact, writing $|\mathcal{D}|_{1}=\left(1+\mathcal{D}^{2}\right)^{1 / 2}$ and $\delta_{1}(T)=\left[|\mathcal{D}|_{1}, T\right]$ we have $\operatorname{dom} \delta^{n}=\operatorname{dom} \delta_{1}^{n}$ for all $n$. 
Proof. Let $f(\mathcal{D})=\left(1+\mathcal{D}^{2}\right)^{1 / 2}-|\mathcal{D}|$, so, as noted above, $f(\mathcal{D})$ extends to a bounded operator in $\mathcal{N}$. Since

$$
\delta_{1}(T)-\delta(T)=[f(\mathcal{D}), T]
$$

is always bounded, dom $\delta=\operatorname{dom} \delta_{1}$. Now $\delta \delta_{1}=\delta_{1} \delta$, so

$$
\begin{aligned}
\delta_{1}^{2}(T)-\delta^{2}(T) & =\delta_{1}\left(\delta_{1}(T)\right)-\delta_{1}(\delta(T))+\delta_{1}(\delta(T))-\delta(\delta(T)) \\
& =\left[f(\mathcal{D}), \delta_{1}(T)\right]+[f(\mathcal{D}), \delta(T)] .
\end{aligned}
$$

Both terms on the right hand side are bounded, so dom $\delta^{2}=d o m \delta_{1}^{2}$. The proof proceeds by induction.

Thus the condition defining $Q C^{k}$ can be replaced by

$$
a,[\mathcal{D}, a] \in \bigcap_{k \geqslant n \geqslant 0} \operatorname{dom} \delta_{1}^{n} \quad \forall a \in \mathcal{A} .
$$

This is important as we do not assume at any point that $|\mathcal{D}|$ is invertible.

If $(\mathcal{A}, \mathcal{H}, \mathcal{D})$ is a $Q C^{k}$ spectral triple, we may endow the algebra $\mathcal{A}$ with the topology determined by the seminorms

$$
a \longrightarrow\left\|\delta^{n}(a)\right\|+\left\|\delta^{n}([\mathcal{D}, a])\right\|, \quad n=0,1,2, \ldots, k
$$

By [85, Lemma 16] we may, without loss of generality, suppose that $\mathcal{A}$ is complete in the resulting topology by completing if necessary. This completion is stable under the holomorphic functional calculus, so we have a sensible spectral theory and $K_{*}(\mathcal{A}) \cong K_{*}(\overline{\mathcal{A}})$ via inclusion, where $\overline{\mathcal{A}}$ is the $C^{*}$-completion of $\mathcal{A}$. A $Q C^{\infty}$ spectral triple is one that is $Q C^{k}$ for all $k=1,2, \ldots$

Observation If $T \in \mathcal{N}$ and $[\mathcal{D}, T]$ is bounded, then $[\mathcal{D}, T] \in \mathcal{N}$.

Proof. Observe that $\mathcal{D}$ is affiliated with $\mathcal{N}$, and so commutes with all projections in the commutant of $\mathcal{N}$, and the commutant of $\mathcal{N}$ preserves the domain of $\mathcal{D}$. Thus if $[\mathcal{D}, T]$ is bounded, it too commutes with all projections in the commutant of $\mathcal{N}$, and these projections preserve the domain of $\mathcal{D}$, and so $[\mathcal{D}, T] \in \mathcal{N}$.

Similar comments apply to $[|\mathcal{D}|, T],\left[\left(1+\mathcal{D}^{2}\right)^{1 / 2}, T\right]$ and combinations such as $\left[\mathcal{D}^{2}, T\right](1+$ $\left.\mathcal{D}^{2}\right)^{-1 / 2}$. We will often simply write $\mathcal{L}^{1}$ for the trace ideal in order to simplify the notation, and denote the norm on $\mathcal{L}^{1}$ by $\|\cdot\|_{1}$. Note that in the case where $\mathcal{N} \neq \mathcal{L}(\mathcal{H}), \mathcal{L}^{1}$ need not be complete in this norm but it is complete in the norm $\|\cdot\|_{1}+\|\cdot\|_{\infty}$. (where $\|\cdot\|_{\infty}$ is the uniform norm).

8.4. Summability and Dimension. Finite summability conditions on a spectral triple give a half-plane where the function

$$
z \mapsto \tau\left(\left(1+\mathcal{D}^{2}\right)^{-z}\right)
$$

is well-defined and holomorphic.

Definition 8.7. If $(\mathcal{A}, \mathcal{H}, \mathcal{D})$ is a $Q C^{\infty}$ spectral triple, we call

$$
p=\inf \left\{a \in \mathbb{R}: \tau\left(\left(1+\mathcal{D}^{2}\right)^{-a / 2}\right)<\infty\right\}
$$

the spectral dimension of $(\mathcal{A}, \mathcal{H}, \mathcal{D})$. 


\section{Spectral Flow}

One of the main motivations for extending the study of spectral triples to the semifinite von Neumann setting is the study of type $I I$ spectral flow (this concept is due to Phillips [80, 81]). Let $\pi: \mathcal{N} \rightarrow \mathcal{N} / \mathcal{K}_{\mathcal{N}}$ be the canonical mapping. A Breuer-Fredholm operator is one that maps to an invertible operator under $\pi$. A full discussion of Breuer-Fredholm theory in a semifinite von Neumann algebra is contained in [22] extending the discussion of the Appendix to [82] and $[14,15]$. As usual $D$ is an unbounded densely defined self-adjoint Breuer-Fredholm operator on $\mathcal{H}$ (meaning $D\left(1+D^{2}\right)^{-1 / 2}$ is bounded and Breuer-Fredholm in $\left.\mathcal{N}\right)$ with $\left(1+D^{2}\right)^{-1 / 2} \in \mathcal{K}_{\mathcal{N}}$. For a unitary $u \in \mathcal{N}$ such that $[D, u]$ is a bounded operator, the path

$$
D_{t}^{u}:=(1-t) D+t u D u^{*}
$$

of unbounded self-adjoint Breuer-Fredholm operators is continuous in the sense that

$$
F_{t}^{u}:=D_{t}^{u}\left(1+\left(D_{t}^{u}\right)^{2}\right)^{-\frac{1}{2}}
$$

is a norm continuous path of self-adjoint Breuer-Fredholm operators in $\mathcal{N}$ [19]. Recall that the Breuer-Fredholm index of a Breuer-Fredholm operator $F$ is defined by

$$
\operatorname{ind}(F)=\tau\left(Q_{\text {kerF }}\right)-\tau\left(Q_{\text {cokerF }}\right)
$$

where $Q_{\text {kerF }}$ and $Q_{\text {cokerF }}$ are the projections onto the kernel and cokernel of $F$.

Definition 9.1. If $\left\{F_{t}\right\}$ is a continuous path of self-adjoint Breuer-Fredholm operators in $\mathcal{N}$, then the definition of the spectral flow of the path, $s f\left(\left\{F_{t}\right\}\right)$ is based on the following sequence of observations in [78] and [81] (see also $[9,11]$ ):

1. While the function $t \mapsto \operatorname{sign}\left(F_{t}\right)$ is typically discontinuous, as is the projection-valued mapping $t \mapsto P_{t}=\frac{1}{2}\left(\operatorname{sign}\left(F_{t}\right)+1\right)$, for $F_{t}=2 P_{t}-1$ with $P_{t}$ the non-negative spectral projection, $t \mapsto \pi\left(P_{t}\right)$ is continuous.

2. If $P$ and $Q$ are projections in $\mathcal{N}$ then $P Q: Q \mathcal{H} \rightarrow P \mathcal{H}$ is a Breuer-Fredholm operator if and only if $\|\pi(P)-\pi(Q)\|<1$ in which case $i n d(P Q) \in \mathbb{R}$ is well-defined.

3. If we partition the parameter interval of $\left\{F_{t}\right\}$ so that the $\pi\left(P_{t}\right)$ do not vary much in norm on each subinterval of the partition then

$$
s f\left(\left\{F_{t}\right\}\right):=\sum_{i=1}^{n} i n d\left(P_{t_{i-1}} P_{t_{i}}\right)
$$

is a well-defined and (path-) homotopy-invariant number which agrees with the usual notion of spectral flow in the type $I_{\infty}$ case.

4. For $D$ and $u$ as above, we define the spectral flow of the path $D_{t}^{u}:=(1-t) D+t u D u^{*}$ to be the spectral flow of the path $F_{t}$ where $F_{t}=D_{t}^{u}\left(1+\left(D_{t}^{u}\right)^{2}\right)^{-\frac{1}{2}}$. We denote this by

$$
s f\left(D, u D u^{*}\right)=s f\left(\left\{F_{t}\right\}\right),
$$

and observe that this is an integer in the $\mathcal{N}=\mathcal{L}(\mathcal{H})$ case and a real number in the general semifinite case.

Special cases of spectral flow in a semifinite von Neumann algebra were discussed in $[77,78$, $79,80,81]$. 
Let $P$ denote the projection onto the nonnegative spectral subspace of $D$. The spectral flow along $\left\{D_{t}^{u}\right\}$ is equal to $s f\left(\left\{F_{t}\right\}\right)$ and by [19] this is the Breuer-Fredholm index of $P u P u^{*}$. (Note that $\operatorname{signF}_{1}^{u}=2 u P u^{*}-1$ and that for this special path we have $P-u P u^{*}$ is compact so $P u P u^{*}$ is certainly Breuer-Fredholm from $u P u^{*} \mathcal{H}$ to $P \mathcal{H}$.) Now, [82, Appendix B], we have ind $\left(P u P u^{*}\right)=\operatorname{ind}(P u P)$.

The operator $P u P$ is known as a generalised Toeplitz operator. Formulae for its index in terms of the Dixmier trace are discussed in Sections 13 and 16. These Dixmier trace formulae follow from the analytic formulae for spectral flow discovered in [55],[19],[20] which we will now explain.

9.1. Spectral Flow Formulae. We now introduce the spectral flow formula of Carey and Phillips, $[19,20]$. This formula starts with a semifinite spectral triple $(\mathcal{A}, \mathcal{H}, \mathcal{D})$ and computes the spectral flow from $\mathcal{D}$ to $u \mathcal{D} u^{*}$, where $u \in \mathcal{A}$ is unitary with $[\mathcal{D}, u]$ bounded, in the case where $(\mathcal{A}, \mathcal{H}, \mathcal{D})$ is of dimension $p \geqslant 1$. Thus for any $n>p$ we have by the extension of Theorem 9.3 of [20] to the case of general semifinite von Neumann algebras (see [23]):

$$
s f\left(\mathcal{D}, u \mathcal{D} u^{*}\right)=\frac{1}{C_{n / 2}} \int_{0}^{1} \tau\left(u\left[\mathcal{D}, u^{*}\right]\left(1+\left(\mathcal{D}+t u\left[\mathcal{D}, u^{*}\right]\right)^{2}\right)^{-n / 2}\right) d t,
$$

with $C_{n / 2}=\int_{-\infty}^{\infty}\left(1+x^{2}\right)^{-n / 2} d x$. This real number $\operatorname{sf}\left(\mathcal{D}, u \mathcal{D} u^{*}\right)$ recovers the pairing of the $K$-homology class $[\mathcal{D}]$ of $\mathcal{A}$ with the $K_{1}(\mathcal{A})$ class $[u]$ (see below). There is a geometric way to view this formula due originally to Getzler [55]. It is shown in [20] that the functional $X \mapsto \tau\left(X\left(1+(\mathcal{D}+X)^{2}\right)^{-n / 2}\right)$ on $\mathcal{N}_{s a}$ determines an exact one-form on an affine space $\mathcal{D}+\mathcal{N}_{s a}$. Thus (15) represents the integral of this one-form along the path $\left\{\mathcal{D}_{t}=(1-t) \mathcal{D}+t u \mathcal{D} u^{*}\right\}$ provided one appreciates that $\dot{\mathcal{D}}_{t}=u\left[\mathcal{D}, u^{*}\right]$ is a tangent vector to this path. Moreover this formula is scale invariant. By this we mean that if we replace $\mathcal{D}$ by $\epsilon \mathcal{D}$, for $\epsilon>0$, in the right hand side of (15), then the left hand side is unchanged as is evident from the definition of spectral flow. This is because spectral flow only involves the phase of $\mathcal{D}$ which is the same as the phase of $\epsilon \mathcal{D}$.

9.2. Relation to Cyclic Cohomology. To place some of the discussion in its correct context and to prepare for later applications we need to discuss some aspects of cyclic cohomology. We will use the normalised $(b, B)$-bicomplex (see [31, 73]).

We introduce the following linear spaces. Let $C_{m}=\mathcal{A} \otimes \overline{\mathcal{A}}^{\otimes m}$ where $\overline{\mathcal{A}}$ is the quotient $\mathcal{A} / \mathbb{C} I$ with $I$ being the identity element of $\mathcal{A}$ and (assuming with no loss of generality that $\mathcal{A}$ is complete in the $\delta$-topology) we employ the projective tensor product. Let $C^{m}=\operatorname{Hom}\left(C_{m}, \mathbb{C}\right)$ be the linear space of continuous multilinear functionals on $C_{m}$. We may define the $(b, B)$ bicomplex using these spaces (as opposed to $C_{m}=\mathcal{A}^{\otimes m+1}$ et cetera) and the resulting cohomology will be the same. This follows because the bicomplex defined using $\mathcal{A} \otimes \overline{\mathcal{A}}^{\otimes m}$ is quasi-isomorphic to that defined using $\mathcal{A} \otimes \mathcal{A}^{\otimes m}$.

A normalised (b, B)-cochain, $\varphi$ is a finite collection of continuous multilinear functionals on $\mathcal{A}$,

$$
\varphi=\left\{\varphi_{m}\right\}_{m=1,2, \ldots, M} \text { with } \varphi_{m} \in C^{m} .
$$

It is a (normalised) (b, B)-cocycle if, for all $m, b \varphi_{m}+B \varphi_{m+2}=0$ where $b: C^{m} \rightarrow C^{m+1}$, $B: C^{m} \rightarrow C^{m-1}$ are the coboundary operators given by 


$$
\begin{aligned}
& \left(B \varphi_{m}\right)\left(a_{0}, a_{1}, \ldots, a_{m-1}\right)=\sum_{j=0}^{m-1}(-1)^{(m-1) j} \varphi_{m}\left(1, a_{j}, a_{j+1}, \ldots, a_{m-1}, a_{0}, \ldots, a_{j-1}\right) \\
& \left(b \varphi_{m-2}\right)\left(a_{0}, a_{1}, \ldots, a_{m-1}\right)= \\
& \sum_{j=0}^{m-2}(-1)^{j} \varphi_{m-2}\left(a_{0}, a_{1}, \ldots, a_{j} a_{j+1}, \ldots, a_{m-1}\right)+(-1)^{m-1} \varphi_{m-2}\left(a_{m-1} a_{0}, a_{1}, \ldots, a_{m-2}\right)
\end{aligned}
$$

We write $(b+B) \varphi=0$ for brevity. Thought of as functionals on $\mathcal{A}^{\otimes m+1}$ a normalised cocycle will satisfy $\varphi\left(a_{0}, a_{1}, \ldots, a_{n}\right)=0$ whenever any $a_{j}=1$ for $j \geqslant 1$. An odd (even) cochain has $\left\{\varphi_{m}\right\}=0$ for $m$ even (odd).

Similarly, a $\left(\mathbf{b}^{\mathbf{T}}, \mathbf{B}^{\mathbf{T}}\right)$-chain, $c$ is a (possibly infinite) collection $c=\left\{c_{m}\right\}_{m=1,2, \ldots}$ with $c_{m} \in C_{m}$. The $(b, B)$-chain $\left\{c_{m}\right\}$ is a $\left(\mathbf{b}^{\mathbf{T}}, \mathbf{B}^{\mathbf{T}}\right)$-cycle if $b^{T} c_{m+2}+B^{T} c_{m}=0$ for all $m$. More briefly, we write $\left(b^{T}+B^{T}\right) c=0$. Here $b^{T}, B^{T}$ are the boundary operators of cyclic homology, and are the transpose of the coboundary operators $b, B$ in the following sense.

The pairing between a $(b, B)$-cochain $\varphi=\left\{\varphi_{m}\right\}_{m=1}^{M}$ and a $\left(b^{T}, B^{T}\right)$-chain $c=\left\{c_{m}\right\}$ is given by

$$
\langle\varphi, c\rangle=\sum_{m=1}^{M} \varphi_{m}\left(c_{m}\right) .
$$

This pairing satisfies

$$
\langle(b+B) \varphi, c\rangle=\left\langle\varphi,\left(b^{T}+B^{T}\right) c\right\rangle .
$$

We have the relations

$$
b^{2}=B^{2}=0=b B+B b=(b+B)^{2}
$$

so that we may define the cyclic cohomology of $\mathcal{A}$ as the cohomology of the total $(b, B)$ complex.

In this survey the main application we will make of the Dixmier trace to cyclic cohomology is the discussion in Section 17 of the formula of A. Connes for the Hochschild class of the Chern character. Here we use the unrenormalised complex $\tilde{C}^{m}=\mathcal{A}^{\otimes m+1}$. The definition of the Hochschild coboundary $b$ on $\tilde{C}^{m}$ involves the same formula (16). The Hochschild cohomology, denoted $H H^{*}\left(\mathcal{A}, \mathcal{A}^{*}\right)$, is then the cohomology of the complex $\left(\tilde{C}^{*}(\mathcal{A}), b\right)$.

The Hochshild boundary on the complex $\tilde{C}_{m}=\mathcal{A}^{\otimes m+1}$ is the operator $b^{T}$. We say that $c$ is a Hochschild cycle if $b^{T} c=0$. When the Hochschild homology is well-defined we denote it by $H H_{*}(\mathcal{A})$.

One can interpret spectral flow (in the type $I$ case) as the pairing between an odd $K$-theory class represented by a unitary $u$, and an odd $K$-homology class represented by $(\mathcal{A}, \mathcal{H}, \mathcal{D}),[31$, Chapter III,IV]. This point of view also makes sense in the general semifinite setting, though one must suitably interpret $K$-homology, [21, 20]. A central feature of [31] is the translation of the $K$-theory pairing to cyclic theory in order to obtain index theorems. One associates to a suitable representative of a $K$-theory class, respectively a $K$-homology class, a class in periodic cyclic homology, respectively a class in periodic cyclic cohomology, called a Chern 
character in both cases. (We will not digress here to discuss the periodic theory and the periodicity operator referring instead to [31] and [57].) The principal result is then

$$
s f\left(\mathcal{D}, u \mathcal{D} u^{*}\right)=\langle[u],[(\mathcal{A}, \mathcal{H}, \mathcal{D})]\rangle=-\frac{1}{\sqrt{2 \pi i}}\left\langle\left[C h_{*}(u)\right],\left[C h^{*}(\mathcal{A}, \mathcal{H}, \mathcal{D})\right]\right\rangle,
$$

where $[u] \in K_{1}(\mathcal{A})$ is a $K$-theory class with representative $u$ and $[(\mathcal{A}, \mathcal{H}, \mathcal{D})]$ is the $K$ homology class of the spectral triple $(\mathcal{A}, \mathcal{H}, \mathcal{D})$. On the right hand side, $C h_{*}(u)$ is the Chern character of $u$. We recall that the Chern character of a unitary $u$ is the following (infinite) collection of odd chains $C h_{2 j+1}(u)$ satisfying $b C h_{2 j+3}(u)+B C h_{2 j+1}(u)=0$,

$$
C h_{2 j+1}(u)=(-1)^{j} j ! u^{*} \otimes u \otimes u^{*} \otimes \cdots \otimes u \quad(2 j+2 \quad \text { entries }) .
$$

We have used the notation $\left[C h_{*}(u)\right]$ for the periodic cyclic homology class. Similarly $\left[C h^{*}(\mathcal{A}, \mathcal{H}, \mathcal{D})\right]$ is the periodic cyclic cohomology class of the Chern character of $(\mathcal{A}, \mathcal{H}, \mathcal{D})$.

\section{The Dixmier trace AND RESIDUES of the ZETA FunCtion}

Many applications of the Dixmier trace rely on being able to calculate it by taking a residue of an associated zeta function. The main result in this direction in the type $I$ case is Proposition IV.2.4 of [31]. Recent advances [25] have extended this to the general semifinite von Neumann setting.

10.1. Preliminaries. First it is useful to have an estimate on the singular values of the operators in $\mathcal{L}^{(1, \infty)}$.

Lemma 10.1. For $T \in \mathcal{L}^{(1, \infty)}$ positive there is a constant $K>0$ such that for each $p \geqslant 1$,

$$
\int_{0}^{t} \mu_{s}(T)^{p} d s \leqslant K^{p} \int_{0}^{t} \frac{1}{(s+1)^{p}} d s .
$$

Proof. By [53, Lemma 2.5 (iv)], for all $0 \leqslant T \in \mathcal{N}$ and all continuous increasing functions $f$ on $[0, \infty)$ with $f(0) \geqslant 0$, we have $\mu_{s}(f(T))=f\left(\mu_{s}(T)\right)$ for all $s>0$. Combining this fact with well-known result of Hardy-Littlewood-Pólya (see e.g. [51], Lemma 4.1), we see that $T_{1} \prec \prec T_{2}, 0 \leqslant T_{1}, T_{2} \in \mathcal{N}$ implies $T_{1}^{p} \prec \prec T_{2}^{p}$ for all $p \in(1, \infty)$. Now, by definition of $\mathcal{L}^{(1, \infty)}$ the singular values of $T$ satisfy $\int_{0}^{t} \mu_{s}(T) d s=O(\log t)$ so that for some $K>0$,

$$
\int_{0}^{t} \mu_{s}(T) d s \leqslant K \int_{0}^{t} \frac{1}{(s+1)} d s, \quad \forall t>0 .
$$

In other words $\mu_{s}(T) \prec \prec K /(1+s)$ and the assertion of lemma follows immediately.

In the next theorem we use dilation invariant states from Theorem 4.7.

Theorem 10.2. (weak* ${ }^{*}$ Karamata theorem) Let $\tilde{\omega} \in L_{\infty}(\mathbb{R})^{*}$ be a dilation invariant state and let $\beta$ be a real valued, increasing, right continuous function on $\mathbb{R}_{+}$which is zero at zero and such that the integral $h(r)=\int_{0}^{\infty} e^{-\frac{t}{r}} d \beta(t)$ converges for all $r>0$ and $C=\tilde{\omega}-\lim _{r \rightarrow \infty} \frac{1}{r} h(r)$ exists. Then

$$
\tilde{\omega}-\lim _{r \rightarrow \infty} \frac{1}{r} h(r)=\tilde{\omega}-\lim _{t \rightarrow \infty} \frac{\beta(t)}{t} .
$$


Remark 10.3. The classical Karamata theorem states, in the notation of the theorem, that if the ordinary $\operatorname{limit}_{\lim _{r \rightarrow \infty}} \frac{1}{r} h(r)=C$ exists then $C=\lim _{t \rightarrow \infty} \frac{\beta(t)}{t}$. The proof of this classical result is obtained by replacing, in the proof of Theorem 10.2, $\tilde{\omega}$-lim throughout by the ordinary limit.

Proof. Let

$$
g(x)= \begin{cases}x^{-1} & \text { for } e^{-1} \leqslant x \leqslant 1 \\ 0 & \text { for } 0 \leqslant x<e^{-1}\end{cases}
$$

so that $g$ is right continuous at $e^{-1}$. Then for $r>0, t \rightarrow e^{-t / r} g\left(e^{-t / r}\right)$ is left continuous at $t=r$. Thus the Riemann-Stieltjes integral $\int_{0}^{\infty} e^{-t / r} g\left(e^{-t / r}\right) d \beta(t)$ exists for each $r>0$. We claim that for any polynomial $p$

$$
\tilde{\omega}-\lim _{r \rightarrow \infty} \frac{1}{r} \int_{0}^{\infty} e^{-t / r} p\left(e^{-t / r}\right) d \beta(t)=C \int_{0}^{\infty} e^{-t} p\left(e^{-t}\right) d t .
$$

To see this first compute for $p(x)=x^{n}$,

$$
\frac{1}{r} \int_{0}^{\infty} e^{-t / r} e^{-n t / r} d \beta(t)=\frac{1}{r} \int_{0}^{\infty} e^{-(n+1) t / r} d \beta(t) .
$$

Therefore

$$
\frac{1}{n+1} \tilde{\omega}-\lim _{r \rightarrow \infty} \frac{1}{r /(n+1)} \int_{0}^{\infty} e^{-(n+1) t / r} d \beta(t)=\frac{C}{n+1}
$$

by dilation invariance of $\tilde{\omega}$. Thus

$$
\tilde{\omega}-\lim _{r \rightarrow \infty} \frac{1}{r} \int_{0}^{\infty} e^{-t / r} e^{-n t / r} d \beta(t)=C \int_{0}^{\infty} e^{-t}\left(e^{-t}\right)^{n} d t .
$$

Since $\tilde{\omega}$ is linear the claim follows for all $p$.

Choose sequences of polynomials $\left\{p_{n}\right\},\left\{P_{n}\right\}$ such that for all $x \in[0,1]$

$$
-1 \leqslant p_{n}(x) \leqslant g(x) \leqslant P_{n}(x) \leqslant 3
$$

and such that $p_{n}$ and $P_{n}$ converge a.e. to $g(x)$. Then since $\tilde{\omega}$ is positive it preserves order:

$$
\begin{gathered}
C \int_{0}^{\infty} e^{-t} p_{n}\left(e^{-t}\right) d t=\tilde{\omega}-\lim _{r \rightarrow \infty} \frac{1}{r} \int_{0}^{\infty} e^{-t / r} p_{n}\left(e^{-t / r}\right) d \beta(t) \leqslant \tilde{\omega}-\lim _{r \rightarrow \infty} \frac{1}{r} \int_{0}^{\infty} e^{-t / r} g\left(e^{-t / r}\right) d \beta(t) \\
\leqslant \ldots \leqslant C \int_{0}^{\infty} e^{-t} P_{n}\left(e^{-t}\right) d t .
\end{gathered}
$$

By the Lebesgue Dominated Convergence Theorem both $\int_{0}^{\infty} e^{-t} p_{n}\left(e^{-t}\right) d t$ and $\int_{0}^{\infty} e^{-t} P_{n}\left(e^{-t}\right) d t$ converge to $\int_{0}^{\infty} e^{-t} g\left(e^{-t}\right) d t$ as $n \rightarrow \infty$. But a direct calculation yields $\int_{0}^{\infty} e^{-t} g\left(e^{-t}\right) d t=1$ and

$$
\int_{0}^{\infty} e^{-t / r} g\left(e^{-t / r}\right) d \beta(t)=\beta(r)
$$

Hence

$$
C=\tilde{\omega}-\lim _{r \rightarrow \infty} \frac{1}{r} \int_{0}^{\infty} e^{-t / r} g\left(e^{-t / r}\right) d \beta(t)=\tilde{\omega}-\lim _{r \rightarrow \infty} \frac{\beta(r)}{r}
$$

Recall that for any $\tau$-measurable operator $T$, the distribution function of $T$ is defined by

$$
\lambda_{t}(T):=\tau\left(\chi_{(t, \infty)}(|T|)\right), \quad t>0
$$


where $\chi_{(t, \infty)}(|T|)$ is the spectral projection of $|T|$ corresponding to the interval $(t, \infty)$ (see $[\mathrm{FK}])$. By Proposition 2.2 of $[\mathrm{FK}]$,

$$
\mu_{s}(T)=\inf \left\{t \geqslant 0: \lambda_{t}(T) \leqslant s\right\}
$$

we infer that for any $\tau$-measurable operator $T$, the distribution function $\lambda_{(\cdot)}(T)$ coincides with the (classical) distribution function of $\mu_{(\cdot)}(T)$. From this formula and the fact that $\lambda$ is right-continuous, we can easily see that for $t>0, s>0$

$$
s \geqslant \lambda_{t} \Longleftrightarrow \mu_{s} \leqslant t \text {. }
$$

Or equivalently,

$$
s<\lambda_{t} \Longleftrightarrow \mu_{s}>t .
$$

Using Remark 3.3 of $[\mathrm{FK}]$ this implies that:

$$
\int_{0}^{\lambda_{t}} \mu_{s}(T) d s=\int_{\left[0, \lambda_{t}\right)} \mu_{s}(T) d s=\tau\left(|T| \chi_{(t, \infty)}(|T|)\right), \quad t>0 .
$$

Lemma 10.4. For $T \in \mathcal{L}^{(1, \infty)}$ and $C>\|T\|_{(1, \infty)}$ we have eventually

$$
\lambda_{\frac{1}{t}}(T) \leqslant C t \log t
$$

Proof. Suppose not and there exists $t_{n} \uparrow \infty$ such that $\lambda_{\frac{1}{t_{n}}}(T)>C t_{n} \log t_{n}$ and so for $s \leqslant C t_{n} \log t_{n}$ we have $\mu_{s}(T) \geqslant \mu_{C t_{n}} \log t_{n}(T)>\frac{1}{t_{n}}$. Then for sufficiently large $n$

$$
\int_{0}^{C t_{n} \log t_{n}} \mu_{s}(T) d s>\frac{1}{t_{n}} \cdot C t_{n} \log t_{n}=C \log t_{n} .
$$

Choose $\delta>0$ with $C-\delta>\|T\|_{(1, \infty)}$. Then for sufficiently large $n$

$$
\begin{gathered}
C \log t_{n}=(C-\delta) \log t_{n}+\delta \log t_{n}>\|T\|_{(1, \infty)} \log \left(C t_{n}\right)+\|T\|_{(1, \infty)} \log \left(\log \left(t_{n}+1\right)\right) \\
=\|T\|_{(1, \infty)} \log \left(C t_{n} \log \left(t_{n}+1\right)\right) .
\end{gathered}
$$

This is a contradiction with the inequality $\int_{0}^{t} \mu_{s}(T) d s \leqslant\|T\|_{(1, \infty)} \log (t+1)$, which holds for any $t>0$ due to the definition of the norm in $\mathcal{L}^{(1, \infty)}$.

An assertion somewhat similar to Proposition 10.5 below was formulated in [83] and supplied with an incorrect proof. We use a different approach.

Proposition 10.5. For $T \in \mathcal{L}^{(1, \infty)}$ positive let $\omega$ be a state on $L_{\infty}\left(\mathbb{R}_{+}^{*}\right)$ satisfying all the conditions of Corollary 4.8. For every $C>0$

$$
\begin{aligned}
& \tau_{\omega}(T)=\omega-\lim _{t \rightarrow \infty} \frac{1}{\log (1+t)} \int_{0}^{t} \mu_{s}(T) d s=\omega-\lim _{t \rightarrow \infty} \frac{1}{\log (1+t)} \tau\left(T \chi_{\left(\frac{1}{t}, \infty\right)}(T)\right) \\
&=\omega-\lim _{t \rightarrow \infty} \frac{1}{\log (1+t)} \int_{0}^{C t \log t} \mu_{s}(T) d s
\end{aligned}
$$

and if one of the $\omega$-limits is a true limit then so are the others. 
Proof. We first note that

$$
\int_{0}^{t} \mu_{s}(T) d s \leqslant \int_{0}^{\lambda_{\frac{1}{t}}(T)} \mu_{s}(T) d s+1, \quad t>0 .
$$

Indeed, the inequality above holds trivially if $t \leqslant \lambda_{\frac{1}{t}}(T)$. If $t>\lambda_{\frac{1}{t}}(T)$, then

$$
\int_{0}^{t} \mu_{s}(T) d s=\int_{0}^{\lambda_{\frac{1}{t}}(T)} \mu_{s}(T) d s+\int_{\lambda_{\frac{1}{t}}(T)}^{t} \mu_{s}(T) d s .
$$

Now $s>\lambda_{\frac{1}{t}}(T)$ implies that $\mu_{s}(T) \leqslant \frac{1}{t}$ so we have

$$
\int_{0}^{t} \mu_{s}(T) d s \leqslant \int_{0}^{\lambda_{\frac{1}{t}}(T)} \mu_{s}(T) d s+\frac{1}{t}\left(t-\lambda_{\frac{1}{t}}(T)\right) \leqslant \int_{0}^{\lambda_{\frac{1}{t}}(T)} \mu_{s}(T) d s+1 .
$$

Using this observation and lemma above we see that for $C>\|T\|_{(1, \infty)}$ and any fixed $\alpha>1$ eventually

$$
\int_{0}^{t} \mu_{s}(T) d s \leqslant \int_{0}^{\lambda_{\frac{1}{t}}(T)} \mu_{s}(T) d s+1 \leqslant \int_{0}^{C t \log t} \mu_{s}(T) d s+1 \leqslant \int_{0}^{t^{\alpha}} \mu_{s}(T) d s+1
$$

and so eventually

$$
\begin{aligned}
\frac{1}{\log (1+t)} \int_{0}^{t} \mu_{s}(T) d s \leqslant & \frac{1}{\log (1+t)}\left(\int_{0}^{\lambda_{\frac{1}{t}}(T)} \mu_{s}(T) d s+1\right) \leqslant \frac{1}{\log (1+t)}\left(\int_{0}^{C t \log t} \mu_{s}(T) d s+1\right) \\
& \leqslant \frac{\log \left(1+t^{\alpha}\right)}{\log (1+t) \log \left(1+t^{\alpha}\right)}\left(\int_{0}^{t^{\alpha}} \mu_{s}(T) d s+1\right) .
\end{aligned}
$$

Taking the $\omega$-limit we get

$$
\begin{aligned}
\tau_{\omega}(T) \leqslant \omega-\lim _{t \rightarrow \infty} & \frac{1}{\log (1+t)} \int_{0}^{\lambda_{\frac{1}{t}}(T)} \mu_{s}(T) d s \leqslant \omega-\lim _{t \rightarrow \infty} \frac{1}{\log (1+t)} \int_{0}^{C t \log t} \mu_{s}(T) d s \\
& \leqslant \omega-\lim _{t \rightarrow \infty} \frac{\alpha}{\log \left(1+t^{\alpha}\right)} \int_{0}^{t^{\alpha}} \mu_{s}(T) d s=\alpha \tau_{\omega}(T)
\end{aligned}
$$

where the last line uses Corollary 4.8 (4). Since this holds for all $\alpha>1$ and using 18 we get the conclusion for $\omega$-limits and $C>\|T\|_{(1, \infty)}$. The assertion for an arbitrary $0<C \leqslant\|T\|_{(1, \infty)}$ follows immediately by noting that for $C^{\prime}>\|T\|_{(1, \infty)}$ one has eventually

$$
\int_{0}^{t} \mu_{s}(T) d s \leqslant \int_{0}^{C t \log t} \mu_{s}(T) d s \leqslant \int_{0}^{C^{\prime} t \log t} \mu_{s}(T) d s .
$$

To see the last assertion of the Proposition suppose that $\lim _{t \rightarrow \infty} \frac{1}{\log (1+t)} \int_{0}^{t} \mu_{s}(T) d s=A$ then by the above argument we get

$$
A \leqslant \liminf _{t \rightarrow \infty} \frac{1}{\log (1+t)} \tau\left(T \chi_{\left(\frac{1}{t}, \infty\right)}(T)\right) \leqslant \limsup _{t \rightarrow \infty} \frac{1}{\log (1+t)} \tau\left(T \chi_{\left(\frac{1}{t}, \infty\right)}(T)\right) \leqslant \alpha A
$$

for all $\alpha>1$ and hence $\lim _{t \rightarrow \infty} \frac{1}{\log (1+t)} \tau\left(T \chi_{\left(\frac{1}{t}, \infty\right)}(T)\right)=A$. On the other hand if the limit $\lim _{t \rightarrow \infty} \frac{1}{\log (1+t)} \tau\left(T \chi_{\left(\frac{1}{t}, \infty\right)}(T)\right)$ exists and equals $B$ say then

$$
\limsup _{t \rightarrow \infty} \frac{1}{\log (1+t)} \int_{0}^{t} \mu_{s}(T) d s \leqslant B \leqslant \alpha \liminf _{t \rightarrow \infty} \frac{1}{\log (1+t)} \int_{0}^{t} \mu_{s}(T) d s
$$


for all $\alpha>1$ and so

$$
\lim _{t \rightarrow \infty} \frac{1}{\log (1+t)} \int_{0}^{t} \mu_{s}(T) d s=B
$$

as well. The remaining claims follow similarly.

10.2. The zeta function and the Dixmier trace. This subsection is motivated by Proposition IV.2.4 of [31]. We will describe several generalisations of this result to the von Neumann setting. Our approach will be analogous though somewhat different to that in [31].

The zeta function of a positive $T \in \mathcal{L}^{(1, \infty)}$ is given by $\zeta(s)=\tau\left(T^{s}\right)$ while for $A \in \mathcal{N}$ we set $\zeta_{A}(s)=\tau\left(A T^{s}\right)$. We are interested in the asymptotic behaviour of $\zeta(s)$ and $\zeta_{A}(s)$ as $s \rightarrow 1$.

Now it is elementary to see that the discussion of singular traces is relevant because by Lemma 10.1 we have for some $K>0$ and all $s>1$

$$
\begin{aligned}
\tau\left(T^{s}\right) & =\int_{0}^{\infty} \mu_{r}\left(T^{s}\right) d r=\int_{0}^{\infty} \mu_{r}(T)^{s} d r \\
& \leqslant \int_{0}^{\infty} \frac{K^{s}}{(1+r)^{s}} d r=\frac{K^{s}}{s-1} .
\end{aligned}
$$

From this it follows that $\left\{(s-1) \tau\left(T^{s}\right) \mid s>1\right\}$ is bounded. Now for $A$ bounded $\mid(s-$ 1) $\tau\left(A T^{s}\right) \mid \leqslant\|A\|(s-1) \tau\left(T^{s}\right)$ so that $(s-1) \tau\left(A T^{s}\right)$ is also bounded and hence for any $\tilde{\omega} \in L_{\infty}(\mathbb{R})^{*}$ satisfying conditions (1), (2) and (3) of Theorem 4.7

$$
\tilde{\omega}-\lim _{r \rightarrow \infty} \frac{1}{r} \tau\left(A T^{1+\frac{1}{r}}\right)
$$

exists.

Here $r \rightarrow \frac{1}{r} \tau\left(A T^{1+\frac{1}{r}}\right)$ is defined as a function on all of $\mathbb{R}$ by extending it to be identically zero for $r<1$. One might like to think of (19) as $\tilde{\omega}-\lim _{s \rightarrow 1}(s-1) \tau\left(A T^{s}\right)$ but this of course does not (strictly speaking) make sense whereas if $\lim _{s \rightarrow 1}(s-1) \tau\left(A T^{s}\right)$ exists then it is $\lim _{r \rightarrow \infty} \frac{1}{r} \tau\left(A T^{1+\frac{1}{r}}\right)$.

In the following theorem we will take $T \in \mathcal{L}^{(1, \infty)}$ positive, $\|T\| \leqslant 1$ with spectral resolution $T=\int \lambda d E(\lambda)$. We would like to integrate with respect to $d \tau(E(\lambda))$; unfortunately, these scalars $\tau(E(\lambda))$ are, in general, all infinite. To remedy this situation, we instead must integrate with respect to the increasing (negative) real-valued function $N_{T}(\lambda)=\tau(E(\lambda)-1)$ for $\lambda>0$. Away from 0 , the increments $\tau(\triangle E(\lambda))$ and $\triangle N_{T}(\lambda)$ are, of course, identical.

Theorem 10.6. For $T \in \mathcal{L}^{(1, \infty)}$ positive, $\|T\| \leqslant 1$ and $\tilde{\omega} \in L_{\infty}(\mathbb{R})^{*}$ satisfying all the conditions of Theorem 4.7, let $\tilde{\omega}=\omega \circ L$ where $L$ is given in section 4 (prior to Definition 4.4), then we have:

$$
\tau_{\omega}(T)=\tilde{\omega}-\lim \frac{1}{r} \tau\left(T^{1+\frac{1}{r}}\right) .
$$

If $\lim _{r \rightarrow \infty} \frac{1}{r} \tau\left(T^{1+\frac{1}{r}}\right)$ exists then

$$
\tau_{\omega}(T)=\lim _{r \rightarrow \infty} \frac{1}{r} \tau\left(T^{1+\frac{1}{r}}\right)
$$

for an arbitrary dilation invariant functional $\omega \in L_{\infty}\left(\mathbb{R}_{+}^{*}\right)^{*}$. 
Proof. By (19) we can apply the weak*-Karamata theorem to $\frac{1}{r} \tau\left(T^{1+\frac{1}{r}}\right)$. First write $\tau\left(T^{1+\frac{1}{r}}\right)=\int_{0^{+}}^{1} \lambda^{1+\frac{1}{r}} d N_{T}(\lambda)$. Thus setting $\lambda=e^{-u}$

$$
\tau\left(T^{1+\frac{1}{r}}\right)=\int_{0}^{\infty} e^{-\frac{u}{r}} d \beta(u)
$$

where $\beta(u)=\int_{u}^{0} e^{-v} d N_{T}\left(e^{-v}\right)=-\int_{0}^{u} e^{-v} d N_{T}\left(e^{-v}\right)$. Since the change of variable $\lambda=e^{-u}$ is strictly decreasing, $\beta$ is, in fact, nonnegative and increasing. By the weak ${ }^{*}$-Karamata theorem applied to $\tilde{\omega} \in L_{\infty}(\mathbb{R})^{*}$

$$
\tilde{\omega}-\lim _{r \rightarrow \infty} \frac{1}{r} \tau\left(T^{1+\frac{1}{r}}\right)=\tilde{\omega}-\lim _{u \rightarrow \infty} \frac{\beta(u)}{u} .
$$

Next with the substitution $\rho=e^{-v}$ we get:

$$
\tilde{\omega}-\lim _{u \rightarrow \infty} \frac{\beta(u)}{u}=\tilde{\omega}-\lim _{u \rightarrow \infty} \frac{1}{u} \int_{e^{-u}}^{1} \rho d N_{T}(\rho) .
$$

Set $f(u)=\frac{\beta(u)}{u}$. We want to make the change of variable $u=\log t$ or in other words to consider $f \circ \log =L f$. We use the discussion in section 4 which tells us that if we start with an $M$ invariant functional $\omega \in L_{\infty}\left(\mathbb{R}_{+}^{*}\right)^{*}$ then the functional $\tilde{\omega}=\omega \circ L$ is $H$ invariant as required by the theorem. Then we have

$$
\tilde{\omega}-\lim _{r \rightarrow \infty} \frac{1}{r} \tau\left(T^{1+\frac{1}{r}}\right)=\tilde{\omega}-\lim _{u \rightarrow \infty} \frac{\beta(u)}{u}=\tilde{\omega}-\lim _{u \rightarrow \infty} f(u)=\omega-\lim _{t \rightarrow \infty} L f(t)=\omega-\lim _{t \rightarrow \infty} \frac{1}{\log t} \int_{1 / t}^{1} \lambda d N_{T}(\lambda) .
$$

Now, by Proposition 10.5

$$
\omega-\lim _{t \rightarrow \infty} \frac{1}{\log t} \int_{1 / t}^{1} \lambda d N_{T}(\lambda)=\omega-\lim _{t \rightarrow \infty} \frac{1}{\log t} \tau\left(\chi_{\left(\frac{1}{t}, 1\right]}(T) T\right)=\tau_{\omega}(T) .
$$

This completes the proof of the first part of the theorem.

The proof of the second part is similar. Using the classical Karamata theorem (see the remark following the statement of Theorem 10.2) we obtain the following analogue of (20):

$$
\lim _{r \rightarrow \infty} \frac{1}{r} \tau\left(T^{1+r}\right)=\lim \frac{\beta(u)}{u}=\lim _{u \rightarrow \infty} \frac{1}{u} \int_{e^{-u}}^{1} \rho d N_{T}(\rho)
$$

Making the substitution $u=\log t$ on the right hand side we have

$$
\lim _{u \rightarrow \infty} \frac{1}{u} \int_{e^{-u}}^{1} \rho d N_{T}(\rho)=\lim _{t \rightarrow \infty} \frac{1}{\log t} \int_{\frac{1}{t}}^{1} \lambda d N_{T}(\lambda)=\tau_{\omega}(T)
$$

where in the last equality we need only dilation invariance of the state $\omega \in L_{\infty}\left(\mathbb{R}_{+}^{*}\right)^{*}$ and not the full list of conditions of Corollary 4.8

The map on positive $T \in \mathcal{L}^{(1, \infty)}$ to $\mathbb{R}$ given by $T \rightarrow \tau_{\omega}(T)$ can be extended by linearity to a $\mathbb{C}$ valued functional on all of $\mathcal{L}^{(1, \infty)}$. Then the functional

$$
A \mapsto \tau_{\omega}(A T)
$$

for $A \in \mathcal{N}$ and fixed $T \in \mathcal{L}^{(1, \infty)}$ is well defined. We intend to study the properties of (21). Part of the interest in this functional stems from the following result (see [26]) as well as the use of the Dixmier trace in noncommutative geometry [31]. 
Lemma 10.7. Let $T \in \mathcal{L}^{(1, \infty)}$, then

(i) For $A \in \mathcal{N}$ we have

$$
\tau_{\omega}(A T)=\tau_{\omega}(T A)
$$

(ii) Assume that $D_{0}$ is an unbounded self adjoint operator affiliated with $\mathcal{N}$ such that $T=\left(1+D_{0}^{2}\right)^{-1 / 2} \in \mathcal{L}^{(1, \infty)}$. If $\left[A_{j},\left|D_{0}\right|\right]$ is a bounded operator for $A_{j} \in \mathcal{N}, j=1,2$ then

$$
\tau_{\omega}\left(A_{1} A_{2} T\right)=\tau_{\omega}\left(A_{2} A_{1} T\right) .
$$

Proof.(i) This is proposition A.2 of [40]. The proof is elementary, first show that $\tau_{\omega}\left(U T U^{*}\right)=$ $\tau_{\omega}(T)$ then use linearity to extend to arbitrary $T \in \mathcal{L}^{(1, \infty)}$. Replace $T$ by $T U$ then use linearity again.

(ii) We remark that $\left[A_{j},\left|D_{0}\right|\right]$ defining a bounded operator means that the $A_{j}$ leave $\operatorname{dom}\left(\left|D_{0}\right|\right)=\operatorname{dom}\left(D_{0}\right)$ invariant and that $\left[A_{j},\left|D_{0}\right|\right]$ is bounded on this domain (see [12, Proposition 3.2.55] and its proof for equivalent but seemingly weaker conditions). As $\left|D_{0}\right|-\left(1+D_{0}^{2}\right)^{1 / 2}$ is bounded, $\left[A_{j},\left(1+D_{0}^{2}\right)^{1 / 2}\right]$ defines a bounded operator whenever $\left[A_{j},\left|D_{0}\right|\right]$ does. As $T^{-1}=\left(1+D_{0}^{2}\right)^{1 / 2}$ and $T: \mathcal{H} \rightarrow \operatorname{dom}\left(T^{-1}\right)$, we see that the formal calculation:

$$
\left[A_{j}, T\right]=A_{j} T-T A_{j}=T\left(T^{-1} A_{j}-A_{j} T^{-1}\right) T=T\left[T^{-1}, A_{j}\right] T
$$

makes sense as an everywhere-defined operator on $\mathcal{H}$. That is,

$$
\left[A_{j}, T\right]=T\left[\left(1+D_{0}^{2}\right)^{1 / 2}, A_{j}\right] T \in\left(\mathcal{L}^{(1, \infty)}\right)^{2} \subseteq \mathcal{L}^{1} .
$$

Then we have, using part (i),

$$
\tau_{\omega}\left(A_{1} A_{2} T\right)=\tau_{\omega}\left(A_{2} A_{1} T\right)-\tau_{\omega}\left(\left[A_{1}, T\right] A_{2}\right) .
$$

So then

$$
\tau_{\omega}\left(A_{1} A_{2} T\right)=\tau_{\omega}\left(A_{2} A_{1} T\right)-\tau_{\omega}\left(T\left[\left(1+D_{0}^{2}\right)^{1 / 2}, A_{1}\right] T A_{2}\right) .
$$

Since the operator in the last term is trace class we are done.

We will consider spectral triples which involves choosing a subalgebra of $\mathcal{N}$ on which (21) will define a trace.

Theorem 10.8. Let $A \in \mathcal{N}, T \geqslant 0, T \in \mathcal{L}^{(1, \infty)}$.

(i) If $\lim _{s \rightarrow 1^{+}}(s-1) \tau\left(A T^{s}\right)$ exists then it is equal to $\tau_{\omega}(A T)$ where we choose $\omega$ as in the proof of Theorem 10.6.

(ii) More generally, if we choose functionals $\omega$ and $\tilde{\omega}$ as in the proof of Theorem 10.6 then

$$
\tilde{\omega}-\lim _{r \rightarrow \infty} \frac{1}{r} \tau\left(A T^{1+\frac{1}{r}}\right)=\tau_{\omega}(A T) .
$$

Proof. For part (i) we first assume that $A$ is self adjoint. Write $A=a^{+}-a^{-}$where $a^{ \pm}$are positive. Choose $\tilde{\omega}$ as in the proof of Theorem 10.6, then

$$
\begin{aligned}
\lim _{s \rightarrow 1^{+}}(s-1) \tau\left(A T^{s}\right) & =\tilde{\omega}-\lim _{r \rightarrow \infty} \frac{1}{r} \tau\left(A T^{1+\frac{1}{r}}\right) \\
& =\tilde{\omega}-\lim _{r \rightarrow \infty} \frac{1}{r} \tau\left(a^{+} T^{1+\frac{1}{r}}\right)-\tilde{\omega}-\lim _{r \rightarrow \infty} \frac{1}{r} \tau\left(a^{-} T^{1+\frac{1}{r}}\right) \\
& =\tau_{\omega}\left(a^{+} T\right)-\tau_{\omega}\left(a^{-} T\right) \\
& =\tau_{\omega}(A T) .
\end{aligned}
$$


Here the third equality uses a technical result (Proposition 3.6) from [25] and then Theorem 10.6. The reduction from the general case to the self-adjoint case now follows in a similar way.

For part (ii), we assume that $A$ is positive. From Lemma 10.7(i) and Theorem 10.6, and Proposition 3.6 of [25] we have

$$
\begin{aligned}
\tau_{\omega}(A T) & =\tau_{\omega}\left(A^{1 / 2} T A^{1 / 2}\right)=\tilde{\omega}-\lim _{r \rightarrow \infty} \frac{1}{r} \tau\left(\left(A^{1 / 2} T A^{1 / 2}\right)^{1+\frac{1}{r}}\right) \\
& =\tilde{\omega}-\lim _{r \rightarrow \infty} \frac{1}{r} \tau\left(A T^{1+\frac{1}{r}}\right) .
\end{aligned}
$$

For general $A$ we reduce to the case $A$ positive as in the proof of part (i).

\section{The heat Semigroup Formula}

This Section is inspired by [31] and again is taken from [25]. We retain the assumption $T \geqslant 0$ throughout and define $e^{-T^{-2}}$ as the operator that is zero on $\operatorname{ker} T$ and on $\operatorname{ker} T^{\perp}$ is defined by the functional calculus. We remark that if $T \geqslant 0, T \in \mathcal{L}^{(p, \infty)}$ for some $p \geqslant 1$ then $e^{-t T^{-2}}$ is trace class for all $t>0$.

Our aim in this Section is to prove the following

Theorem 11.1. If $A \in \mathcal{N}, T \geqslant 0, T \in \mathcal{L}^{(1, \infty)}$ then,

$$
\omega-\lim _{\lambda \rightarrow \infty} \lambda^{-1} \tau\left(A e^{-\lambda^{-2} T^{-2}}\right)=\Gamma(3 / 2) \tau_{\omega}(A T)
$$

for $\omega \in L_{\infty}\left(\mathbb{R}_{+}^{*}\right)^{*}$ satisfying the conditions of Corollary 4.8.

Let $\zeta_{A}\left(p+\frac{1}{r}\right)=\tau\left(A T^{p+\frac{1}{r}}\right)$. Notice that $\frac{1}{2} \Gamma\left(\frac{p}{2}\right) \tilde{\omega}-\lim _{r \rightarrow \infty} \frac{1}{r} \zeta_{A}\left(p+\frac{1}{r}\right)$ always exists. Hence we can reduce the hard part of the proof of Theorem 11.1 to the following preliminary result.

Proposition 11.2. If $A \in \mathcal{N}, A \geqslant 0, T \geqslant 0, T \in \mathcal{L}^{(p, \infty)}, 1 \leqslant p<\infty$ then, choosing $\omega$ and $\tilde{\omega}$ as in the proof of Theorem 10.6, we have

$$
\omega-\lim _{\lambda \rightarrow \infty} \frac{1}{\lambda} \tau\left(A e^{-T^{-2} \lambda^{-2 / p}}\right)=\frac{1}{2} \Gamma\left(\frac{p}{2}\right) \tilde{\omega}-\lim _{r \rightarrow \infty} \frac{1}{r} \zeta_{A}\left(p+\frac{1}{r}\right) .
$$

Proof. We have, using the Laplace transform,

$$
T^{s}=\frac{1}{\Gamma(s / 2)} \int_{0}^{\infty} t^{s / 2-1} e^{-t T^{-2}} d t
$$

Then

$$
\zeta_{A}(s)=\tau\left(A T^{s}\right)=\frac{1}{\Gamma(s / 2)} \int_{0}^{\infty} t^{s / 2-1} \tau\left(A e^{-t T^{-2}}\right) d t
$$

Make the change of variable $t=1 / \lambda^{2 / p}$ so that the preceding formula becomes

$$
\frac{p}{2} \Gamma(s / 2) \zeta_{A}(s)=\int_{0}^{\infty} \lambda^{-\frac{s}{p}-1} \tau\left(A e^{-\lambda^{-2 / p} T^{-2}}\right) d \lambda .
$$

We split this integral into two parts, $\int_{0}^{1}$ and $\int_{1}^{\infty}$ and call the first integral $R(r)$ where $s=p+\frac{1}{r}$. Then

$$
R(r)=\int_{0}^{1} \lambda^{-\frac{1}{p r}-2} \tau\left(A e^{-\lambda^{-2 / p} T^{-2}}\right) d \lambda=\int_{1}^{\infty} t^{\frac{p}{2}+\frac{1}{2 r}-1} \tau\left(A e^{-t T^{-2}}\right) d t .
$$


The integrand decays exponentially in $t$ as $t \rightarrow \infty$ because $T^{-2} \geqslant\left\|T^{2}\right\|^{-1} \mathbf{1}$ so that

$$
\tau\left(A e^{-t T^{-2}}\right) \leqslant \tau\left(A e^{-T^{-2}} e^{-\frac{t-1}{\left\|T^{2}\right\|}}\right) .
$$

Then we can conclude that $R(r)$ is bounded independently of $r$ and so $\lim _{r \rightarrow \infty} \frac{1}{r} R(r)=0$. For the other integral the change of variable $\lambda=e^{\mu}$ gives

$$
\int_{1}^{\infty} \lambda^{-\frac{1}{p r}-2} \tau\left(A e^{-\lambda^{-2 / p} T^{-2}}\right) d \lambda=\int_{0}^{\infty} e^{-\frac{\mu}{p r}} d \beta(\mu)
$$

where $\beta(\mu)=\int_{0}^{\mu} e^{-v} \tau\left(A e^{-e^{-\frac{2}{p} v} T^{-2}}\right) d v$. Hence we can now write

$$
\frac{p}{2} \Gamma\left(\left(p+\frac{1}{r}\right) / 2\right) \zeta_{A}\left(p+\frac{1}{r}\right)=\int_{0}^{\infty} e^{-\frac{\mu}{p r}} d \beta(\mu)+R(r) .
$$

Now consider

$$
\frac{p}{2} \tilde{\omega}-\lim _{r \rightarrow \infty} \frac{1}{r} \Gamma\left(\frac{p}{2}+\frac{p}{2 r}\right) \zeta_{A}\left(p+\frac{1}{r}\right)=\frac{p}{2} \Gamma(p / 2) \tilde{\omega}-\lim _{r \rightarrow \infty} \frac{1}{r} \zeta_{A}\left(p+\frac{1}{r}\right) .
$$

Then

$$
\frac{p}{2} \Gamma(p / 2) \tilde{\omega}-\lim _{r \rightarrow \infty} \frac{1}{r} \zeta_{A}\left(p+\frac{1}{r}\right)=p \tilde{\omega}-\lim _{r \rightarrow \infty} \frac{1}{p r} \int_{0}^{\infty} e^{-\mu / p r} d \beta(\mu)
$$

(remembering that the term $\frac{1}{r} R(r)$ has limit zero as $r \rightarrow \infty$ ). By dilation invariance and Theorem 10.2 we then have

$$
\frac{p}{2} \Gamma(p / 2) \tilde{\omega}-\lim _{r \rightarrow \infty} \frac{1}{r} \zeta_{A}\left(p+\frac{1}{r}\right)=p \tilde{\omega}-\lim _{\mu \rightarrow \infty} \frac{\beta(\mu)}{\mu} .
$$

Making the change of variable $\lambda=e^{v}$ in the expression for $\beta(\mu)$ we get

$$
\frac{\beta(\mu)}{\mu}=\frac{1}{\mu} \int_{1}^{e^{\mu}} \lambda^{-2} \tau\left(A e^{-T^{-2} \lambda^{-2 / p}}\right) d \lambda
$$

Make the substitution $\mu=\log t$ so the RHS becomes

This is the Cesaro mean of

$$
\frac{1}{\log t} \int_{1}^{t} \lambda^{-2} \tau\left(A e^{-T^{-2} \lambda^{-2 / p}}\right) d \lambda=g_{1}(t)
$$

$$
g_{2}(\lambda)=\frac{1}{\lambda} \tau\left(A e^{-T^{-2} \lambda^{-2 / p}}\right) .
$$

So as we chose $\omega \in L_{\infty}\left(\mathbb{R}_{+}^{*}\right)^{*}$ satisfying Corollary 4.8 , we have $\omega\left(g_{1}\right)=\omega\left(g_{2}\right)$. Recalling that we choose $\tilde{\omega}$ to be related to $\omega$ as in Theorem 10.6 and so using (22) we obtain

$$
\omega-\lim _{\lambda \rightarrow \infty} \frac{1}{\lambda} \tau\left(A e^{-T^{-2} \lambda^{-2 / p}}\right)=\frac{1}{2} \Gamma\left(\frac{p}{2}\right) \tilde{\omega}-\lim _{r \rightarrow \infty} \frac{1}{r} \zeta_{A}\left(p+\frac{1}{r}\right) .
$$

To prove the theorem consider first the case where $A$ is bounded, $A \geqslant 0$ and use the Proposition 11.2 and Theorem 10.8 to assert that

$$
\Gamma(3 / 2) \tau_{\omega}(A T)=\Gamma(3 / 2) \tilde{\omega}-\lim _{r \rightarrow \infty} \frac{1}{r} \tau\left(A T^{1+\frac{1}{r}}\right)=\omega-\lim _{\lambda \rightarrow \infty} \lambda^{-1} \tau\left(A e^{-\lambda^{-2} T^{-2}}\right) .
$$

Then for self adjoint $A$ write $A=a^{+}-a^{-}$where $a^{ \pm}$are positive so that

$$
\begin{gathered}
\Gamma(3 / 2) \tau_{\omega}(A T)=\Gamma(3 / 2)\left(\tau_{\omega}\left(a^{+} T\right)-\tau_{\omega}\left(a^{-} T\right)\right) \\
=\omega-\lim _{\lambda \rightarrow \infty} \lambda^{-1} \tau\left(a^{+} e^{-\lambda^{-2} T^{-2}}\right)-\omega-\lim _{\lambda \rightarrow \infty} \lambda^{-1} \tau\left(a^{-} e^{-\lambda^{-2} T^{-2}}\right)
\end{gathered}
$$




$$
=\omega-\lim _{\lambda \rightarrow \infty} \lambda^{-1} \tau\left(A e^{-\lambda^{-2} T^{-2}}\right) .
$$

We can extend to general bounded $A$ by a similar argument.

\section{The CASE OF $p>1$}

The results of the previous sections have analogues for $p>1$. Some arguments are simpler due to the fact that the singular values satisfy for $T \in \mathcal{L}^{(p, \infty)}$ for $p>1, T \geqslant 0$ the asymptotic estimate $\mu_{s}(T)=O\left(\frac{1}{s^{1 / p}}\right)$. Moreover $\tau\left(T^{p+\frac{1}{r}}\right)=\int_{0}^{1} \lambda^{p+1 / r} d N_{T}(\lambda)$ where $N_{T}(\lambda)=\tau(E(\lambda)-1)$ for $\lambda>0$ where $T=\int \lambda d E(\lambda)$ is the spectral resolution for $T$.

We now establish some $\mathcal{L}^{(p, \infty)}$ versions of our previous results.

Lemma 12.1. For $T \in \mathcal{L}^{(p, \infty)}$ and $\omega$ and $\tilde{\omega}$ as in the proof of theorem 10.6 we have

$$
p \tau_{\omega}\left(T^{p}\right)=\tilde{\omega}-\lim _{r \rightarrow \infty} \frac{1}{r} \tau\left(T^{p+\frac{1}{r}}\right) .
$$

Proof. Set $\lambda=e^{-u / p}$ so that

$$
\frac{1}{r} \tau\left(T^{p+\frac{1}{r}}\right)=p \frac{1}{p r} \int_{0}^{\infty} e^{-u / r p} d \beta(u)
$$

where $\beta(u)=\int_{0}^{u} e^{-v} d N_{T}\left(e^{-v / p}\right)$. So using dilation invariance:

$$
\tilde{\omega}-\lim _{r \rightarrow \infty} \frac{1}{r} \tau\left(T^{p+\frac{1}{r}}\right)=p \tilde{\omega}-\lim _{r \rightarrow \infty} \frac{1}{p r} \int_{0}^{1} e^{-u / p r} d \beta(u)=p \tilde{\omega}-\lim _{u \rightarrow \infty} \frac{\beta(u)}{u}
$$

by the weak*-Karamata Theorem 10.2. Reasoning as in the proof of Theorem 10.6 and substituting $\lambda=e^{-v / p}$ and $u=\log t$ we have

$$
\begin{gathered}
\tilde{\omega}-\lim _{u \rightarrow \infty} \frac{\beta(u)}{u}=\omega-\lim _{t \rightarrow \infty} \frac{1}{\log t} \int_{t^{-1 / p}}^{1} \lambda^{p} d N_{T}(\lambda) \\
=\omega-\lim _{t \rightarrow \infty} \frac{1}{\log t} \tau\left(\chi_{\left(\frac{1}{t}, \infty\right)}\left(T^{p}\right) T^{p}\right)=\tau_{\omega}\left(T^{p}\right) .
\end{gathered}
$$

Corollary 12.2. Let $T \geqslant 0, T \in \mathcal{L}^{(p, \infty)}$ then

$$
\omega-\lim _{\lambda \rightarrow \infty} \frac{1}{\lambda} \tau\left(e^{-T^{-2} \lambda^{-2 / p}}\right)=\Gamma\left(1+\frac{p}{2}\right) \tilde{\omega}-\lim _{r \rightarrow \infty} \frac{1}{r} \tau\left(T^{p+\frac{1}{r}}\right) .
$$

Proof. Combine Proposition 11.2 and Lemma 12.1.

The $\mathcal{L}^{(p, \infty)}$ version of Theorem 10.8 and the following result of Connes' are proved by following the same methods as for $p=1$. One needs a number of straightforward extensions of various technical results as described in Section 5 of [25].

Theorem 12.3. If $A$ is bounded, $T \geqslant 0, T \in \mathcal{L}^{(p, \infty)}$ for $p \geqslant 1$

$$
\omega-\lim _{\lambda \rightarrow \infty} \lambda^{-1} \tau\left(A e^{-\lambda^{-2 / p} T^{-2}}\right)=\Gamma(1+p / 2) \tau_{\omega}\left(A T^{p}\right) .
$$

Finally it is now straightforward to obtain the following result. 
Theorem 12.4. If $A$ is bounded, $T \geqslant 0, T \in \mathcal{L}^{(p, \infty)}$ and

$$
\lim _{s \rightarrow p^{+}}(s-p) \tau\left(A T^{s}\right)
$$

exists then it is equal to $p \tau_{\omega}\left(A T^{p}\right)$.

\section{Generalised Toeplitz operators And their index}

The index theory of generalised Toeplitz operators has a long history which may be traced in part from [27] and [70]. We will explain how the results established in earlier sections may be used to contribute to this theory.

As before $P$ denotes the projection onto the nonnegative spectral subspace of an unbounded self adjoint operator $\mathcal{D}_{0}$ (with bounded inverse) and we are interested in the Breuer-Fredholm index of the operator $P u P$ acting on $P \mathcal{H}$. When $\mathcal{N}=\mathcal{L}(\mathcal{H})$ and $\mathcal{D}_{0}$ is part of an $\mathcal{L}^{(1, \infty)}$ summable spectral triple the most general such theorem in this type $I$ situation is that due to [40] who show show that

$$
\operatorname{ind}(P u P)=\frac{1}{2} \tau_{\omega}\left(u\left[\mathcal{D}_{0}, u^{*}\right]\left|\mathcal{D}_{0}\right|^{-1}\right)
$$

We now show that this formula holds when $\mathcal{N}$ is a general semifinite von Neumann algebra.

Remark. Of course [40] consider a much more general situation which would apply for example in the case where $\mathcal{D}_{0}$ is part of an $\mathcal{L}^{(p, \infty)}$ summable spectral triple. The authors obtain a very general formula for the index of $P u P$ in terms of sums of residues of zeta functions constructed from $\mathcal{D}_{0}$ under certain assumptions on the analytic properties of these zeta functions. Some, but not all, of the zeta functions in their formula are Dixmier traces. We will not attempt to describe this result here as there is an excellent overview in the article [64]. The theorem in [40] is proved for the type $I$ case and its generalisation to the semifinite von Neumann algebra setting is achieved in $[21,22]$.

We need a preliminary result, [25] Lemma 6.1.

Lemma 13.1. Let $D_{0}$ be an unbounded self-adjoint operator affiliated with $\mathcal{N}$ so that $(1+$ $\left.D_{0}^{2}\right)^{-1 / 2}$ is in $\mathrm{cl}^{(1, \infty)}$. Let $A_{t}$ and $B$ be in $\mathcal{N}$ for $t \in[0,1]$ with $A_{t}$ self-adjoint and $t \mapsto A_{t}$ continuous. Let $D_{t}=D_{0}+A_{t}$ and let $p$ be a real number with $1<p<4 / 3$. Then, the quantity

$$
\tau\left(B\left(1+D_{0}^{2}\right)^{-p / 2}-B\left(1+D_{t}^{2}\right)^{-p / 2}\right)
$$

is uniformly bounded independent of $t \in[0,1]$ and $p \in(1,4 / 3)$.

Theorem 13.2. Let $\left(\mathcal{N}, \mathcal{D}_{0}\right)$ be a $\mathcal{L}^{(1, \infty)}$-summable Breuer-Fredholm module for the unital Banach $*$-algebra, $\mathcal{A}$, and let $u \in \mathcal{A}$ be a unitary such that $\left[\mathcal{D}_{0}, u\right]$ is bounded. Let $P$ be the projection on the non-negative spectral subspace of $\mathcal{D}_{0}$. Then with $\omega$ chosen as in Corollary 
4.8,

$$
\begin{aligned}
\operatorname{ind}(\operatorname{PuP})=\operatorname{sf}\left(\mathcal{D}_{0}, u \mathcal{D}_{0} u^{*}\right) & =\lim _{p \rightarrow 1^{+}} \frac{1}{2}(p-1) \tau\left(u\left[\mathcal{D}_{0}, u^{*}\right]\left(1+\mathcal{D}_{0}^{2}\right)^{-p / 2}\right) \\
& =\frac{1}{2} \tau_{\omega}\left(u\left[\mathcal{D}_{0}, u^{*}\right]\left(1+\mathcal{D}_{0}^{2}\right)^{-1 / 2}\right) \\
& =\frac{1}{2} \tau_{\omega}\left(u\left[\mathcal{D}_{0}, u^{*}\right]\left|\mathcal{D}_{0}\right|^{-1}\right)
\end{aligned}
$$

where the last equality only holds if $\mathcal{D}_{0}$ has a bounded inverse.

Remark 13.3. (1) The equality

$$
\operatorname{ind}(P u P)=\frac{1}{2} \tau_{\omega}\left(u\left[\mathcal{D}_{0}, u^{*}\right]\left|\mathcal{D}_{0}\right|^{-1}\right)
$$

proved above should be compared with Theorem IV.2.8 of [31]. In the case where $\mathcal{N}=\mathcal{L}(\mathcal{H})$ the RHS of (23) is a Hochschild 1 - cocycle on $\mathcal{A}$ which is known to equal the Chern character of the $\mathcal{L}^{(1, \infty)}$-summable Fredholm module $\left(\mathcal{A}, \mathcal{D}_{0}, \mathcal{H}\right)$.

(2) Since any 1 -summable module is clearly a $\mathcal{L}^{(1, \infty)}$-summable module, the theorem implies that any unbounded 1-summable module must have a trivial pairing with $K_{1}(\mathcal{A})$ and is therefore uninteresting from the homological point of view.

Proof. Let $\mathcal{D}_{t}^{u}=\mathcal{D}_{0}+t u\left[\mathcal{D}_{0}, u^{*}\right], t \in[0,1]$ then by equation (2) of Section 2.1 (of [20]) we have for each $p>1$ that

$$
\operatorname{ind}(P u P)=\frac{1}{C_{p / 2}} \int_{0}^{1} \tau\left(u\left[\mathcal{D}_{0}, u^{*}\right]\left(1+\left(\mathcal{D}_{t}^{u}\right)^{2}\right)^{-p / 2}\right) d t .
$$

Now, by Lemma 13.1, we have that

$$
\left|\tau\left(u\left[\mathcal{D}_{0}, u^{*}\right]\left[\left(1+\left(\mathcal{D}_{t}^{u}\right)^{2}\right)^{-p / 2}-\left(1+\mathcal{D}_{0}^{2}\right)^{-p / 2}\right]\right)\right|
$$

is uniformly bounded independent of $t$ and $p$ for $1<p<4 / 3$. Since, $\tilde{C}_{p / 2} \rightarrow \infty$ as $p \rightarrow 1^{+}$, we see that:

$$
\begin{aligned}
& \left|\operatorname{ind}(P u P)-\frac{1}{C_{p / 2}} \tau\left(u\left[\mathcal{D}_{0}, u^{*}\right]\left(1+\mathcal{D}_{0}^{2}\right)^{-p / 2}\right)\right| \\
= & \left|\frac{1}{C_{p / 2}} \int_{0}^{1} \tau\left(u\left[\mathcal{D}_{0}, u^{*}\right]\left(1+\left(\mathcal{D}_{t}^{u}\right)^{2}\right)^{-p / 2}\right) d t-\frac{1}{C_{p / 2}} \int_{0}^{1} \tau\left(u\left[\mathcal{D}_{0}, u^{*}\right]\left(1+\mathcal{D}_{0}^{2}\right)^{-p / 2}\right) d t\right| \\
\leqslant & \frac{1}{C_{p / 2}} \int_{0}^{1}\left|\tau\left(u\left[\mathcal{D}_{0}, u^{*}\right]\left[\left(1+\left(\mathcal{D}_{t}^{u}\right)^{2}\right)^{-p / 2}-\left(1+\mathcal{D}_{0}^{2}\right)^{-p / 2}\right]\right)\right| d t \\
\leqslant & \text { Constant } / C_{p / 2} \rightarrow 0
\end{aligned}
$$

Now, it is elementary that as $p \rightarrow 1^{+}$

$$
\frac{2}{p-1}=\int_{|x| \geqslant 1}\left(\frac{1}{|x|}\right)^{p} d x \sim \int_{-\infty}^{\infty}\left(\frac{1}{\sqrt{1+x^{2}}}\right)^{p} d x=\tilde{C}_{p / 2} .
$$

This ends the proof of the first equality while the second equality follows from Theorem 10.8(i). 
The third equality follows from

$$
\left(\sqrt{1+\mathcal{D}_{0}^{2}}\right)^{-1}-\left|\mathcal{D}_{0}\right|^{-1}=\left(\sqrt{1+\mathcal{D}_{0}^{2}}\right)^{-1}\left|\mathcal{D}_{0}\right|^{-1}\left(\sqrt{1+\mathcal{D}_{0}^{2}}+\left|\mathcal{D}_{0}\right|\right)^{-1}
$$

\section{Non-SMOOTh FOLIATIONS AND PSEUdO-DIFFERENTIAL OPERATORS}

In this Section we give a further application of the earlier results following [10] and [83]. The main aim of Prinzis' thesis [83] was to establish a Wodzicki residue formula for the Dixmier trace of certain pseudo-differential operators associated to actions of $\mathbb{R}^{n}$ on a compact space $X$. This was greatly generalised in [10], however to cover that paper in detail would require an extensive discussion of foliations, thus we restrict ourselves here to a synopsis of the simple case in [83].

The set-up is the group-measure space construction of Murray-von Neumann. Thus $X$ is a compact space equipped with a probability measure $\nu$ and a continuous free minimal ergodic action $\alpha$ of $\mathbb{R}^{n}$ on $X$ leaving $\nu$ invariant. We write the action as $x \rightarrow t$. $x$ for $x \in X$ and $t \in \mathbb{R}^{n}$. Then the crossed product $L_{\infty}(X, \nu) \times_{\alpha} \mathbb{R}^{n}$ is a type $I I$ factor contained in the bounded operators on $L^{2}\left(\mathbb{R}^{n}, L^{2}(X, \nu)\right)$. We describe the construction. For a function $f \in L^{1}\left(\mathbb{R}, L_{\infty}(X, \nu)\right) \subset L_{\infty}(X, \nu) \times_{\alpha} \mathbb{R}^{n}$ the action of $f$ on a vector $\xi$ in $L^{2}\left(\mathbb{R}^{n}, L^{2}(X, \nu)\right)$ is defined by twisted left convolution as follows:

$$
(\tilde{\pi}(f) \xi)(s)=\int_{\mathbb{R}^{n}} \alpha_{s}^{-1}(f(t)) \xi(s-t) d t
$$

Here $f(t)$ is a function on $X$ acting as a multiplication operator on $L^{2}(X, \nu)$. The twisted convolution algebra

$$
L^{1}\left(\mathbb{R}^{n}, L_{\infty}(X, \nu)\right) \cap L^{2}\left(\mathbb{R}^{n}, L^{2}(X, \nu)\right)
$$

is a dense subspace of $L^{2}\left(\mathbb{R}^{n}, L^{2}(X, \nu)\right)$ and there is a canonical faithful, normal, semifinite trace, $T r$, on the von Neumann algebra that it generates. This von Neumann algebra is

$$
\mathcal{N}=\left(\tilde{\pi}\left(L_{\infty}(X, \nu) \times_{\alpha} \mathbb{R}^{n}\right)\right)^{\prime \prime} .
$$

For functions $f, g: \mathbb{R}^{n} \rightarrow L_{\infty}(X)$ which are in $L^{2}\left(\mathbb{R}^{n}, L^{2}(X, \nu)\right)$ and whose twisted left convolutions $\tilde{\pi}(f), \tilde{\pi}(g)$ define bounded operators on $L^{2}\left(\mathbb{R}^{n}, L^{2}(X, \nu)\right)$, this trace is given by:

$$
\operatorname{Tr}\left(\tilde{\pi}(f)^{*} \tilde{\pi}(g)\right)=\int_{\mathbb{R}^{n}} \int_{X} f(t, x) g(t, x)^{*} d \nu(x) d t
$$

where we think of $f, g$ as functions on $\mathbb{R}^{n} \times X$.

Identify $L^{2}\left(\mathbb{R}^{n}\right)$ with $L^{2}\left(\mathbb{R}^{n}\right) \otimes 1 \subset L^{2}\left(\mathbb{R}, L^{2}(X, \nu)\right)$ then any scalar-valued function $f$ on $\mathbb{R}^{n}$ which is the Fourier transform $f=\widehat{g}$ of a bounded $L^{2}$ function, $g$ will satisfy $f \in$ $L^{2}\left(\mathbb{R}, L^{2}(X, \nu)\right)$ and $\tilde{\pi}(f)$ will be a bounded operator.

Pseudo-differential operators are defined in terms of their symbols. A smooth symbol of order $m$ is a function $a: X \times \mathbb{R}^{n} \rightarrow \mathbb{C}$ such that for each $x \in X \quad a_{x}$, defined by $a_{x}(t, \xi)=a(t . x, \xi)$, satisfies

(1) $\sup \left\{\left|\partial_{\xi}^{\beta} \partial_{t}^{\gamma} a_{x}(t, \xi)(1+|\xi|)^{-m+|\beta|}\right|(t, \xi) \in \mathbb{R}^{n} \times \mathbb{R}^{n}, \beta, \gamma \in \mathbb{N}^{n},|\beta|+|\gamma| \leqslant M\right\}<\infty$ for all $M \in \mathbb{N}$ 
(2) $\xi \rightarrow a_{x}(0, \xi)$ is a smooth function on $\mathbb{R}^{n}$ into the space $\mathcal{C}^{\infty}(X)$, the set of continuous functions $f$ on $X$ such that $t \rightarrow(x \rightarrow f(t . x))$ is smooth on $\mathbb{R}^{n}$.

Each symbol $a$ defines a pseudo-differential operator $O p(a)$ on $C(X) \otimes C_{c}^{\infty}\left(\mathbb{R}^{n}\right)$ (where $C_{c}^{\infty}\left(\mathbb{R}^{n}\right)$ is the space of smooth functions of compact support) by

$$
O p(a) f(x, t)=\frac{1}{(2 \pi)^{n}} \int_{\mathbb{R}^{n}} e^{i t \xi} a(t . x, \xi) \hat{f}(x, \xi) d \xi, \quad f \in C(X) \otimes C_{c}^{\infty}\left(\mathbb{R}^{n}\right) .
$$

The principal symbol of a pseudo-differential operator $A$ on $X$ is the limit

$$
\sigma_{m}(A)(x, \xi)=\lim _{\lambda \rightarrow \infty} \frac{a(x, \lambda \xi)}{\lambda^{m}}, \quad(x, \xi) \in X \times \mathbb{R}^{n} \backslash\{0\}
$$

if it exists. We say $A$ is elliptic if its symbol $a$ is such that $a_{x}$ is elliptic (that is invertible for all sufficiently large $\xi$ ) for all $x \in X$. Prinzis studies invertible positive elliptic pseudo-differential operators $A$ with a principal symbol. Henceforth we will only consider such operators. The zeta function of such an operator is $\zeta(z)=\tau\left(A^{z}\right)$ and this exists because $A^{z}$ is in the trace class in $\mathcal{N}$ [83] for $\operatorname{Re} z<-n / m$. Prinzis shows that

$$
\lim _{x \rightarrow-\frac{n}{m}-}\left(x+\frac{n}{m}\right) \zeta(x)=-\frac{1}{(2 \pi)^{n} m} \int_{X \times S^{n-1}} \sigma_{m}(A)(x, \xi)^{-\frac{n}{m}} d \nu(x) d \xi
$$

and that $A^{-\frac{n}{m}} \in \mathcal{L}^{(1, \infty)}(\mathcal{N}, \tau)$.

Now note that (24) combined with Theorem 12.4 implies that we have the relation

$$
\tau_{\omega}\left(A^{-\frac{n}{m}}\right)=\frac{1}{(2 \pi)^{n} n} \int_{X \times S^{n-1}} \sigma_{m}(A)(x, \xi)^{-\frac{n}{m}} d \nu(x) d \xi .
$$

In other words we have a type $I I$ Wodzicki residue for evaluating the Dixmier trace of these pseudo-differential operators.

Now, [10] considers the case of measured foliations in the sense of [36]. Thus the leaves of the foliation are no longer given by an action of $\mathbb{R}^{n}$ as above but are more general submanifolds. The main result of [10] on this topic is a formula for the Dixmier trace of certain pseudodifferential operators in terms of a local residue where the latter is a generalisation of the Wodzicki residue to the foliation setting.

\section{The Algebra of Almost PeRiodic FunCtions.}

This section is adapted from [9].

15.1. Almost periodic pseudodifferential operators. We review Shubin's [89] study of the index theory of differential operators with almost periodic coefficients which extends ideas of [27]. Recall that a trigonometric function is a finite linear combination of exponential functions $e_{\xi}: x \mapsto e^{i<x, \xi>}$. The space $\operatorname{Trig}\left(\mathbb{R}^{n}\right)$ of trigonometric functions is a $*$-subalgebra of the $C^{*}$-algebra $C_{b}\left(\mathbb{R}^{n}\right)$ of continuous bounded functions. The uniform closure of $\operatorname{Trig}\left(\mathbb{R}^{n}\right)$ is called the algebra of almost periodic functions and denoted $\mathcal{A} P\left(\mathbb{R}^{n}\right)$. This $C^{*}$-algebra is isomorphic to the algebra of continuous functions on $\mathbb{R}_{B}^{n}$, the Bohr compactification of $\mathbb{R}^{n}$. Addition in $\mathbb{R}^{n}$ extends to $\mathbb{R}_{B}^{n}$ which is a compact abelian group containing $\mathbb{R}^{n}$ as a 
dense subgroup. The normalized Haar measure $\alpha_{B}$ on $\mathbb{R}_{B}^{n}$ is such that the family $\left(e_{\xi}\right)_{\xi \in \mathbb{R}^{n}}$ is orthonormal. The measure $\alpha_{B}$ is given for any almost periodic function $f$ on $\mathbb{R}^{n}$ by:

$$
\alpha_{B}(f):=\lim _{T \rightarrow+\infty} \frac{1}{(2 T)^{n}} \int_{(-T, T)^{n}} f(x) d x .
$$

Using $\alpha_{B}$ one defines the Hilbert space completion $L^{2}\left(\mathbb{R}_{B}^{n}\right)$ of $\operatorname{Trig}\left(\mathbb{R}^{n}\right)$. This Hilbert space has an orthonormal basis given by $\left(e_{\xi}\right)_{\xi \in \mathbb{R}^{n}}$. The Fourier transform $\mathcal{F}_{B}: \ell^{2}\left(\mathbb{R}_{d}^{n}\right) \longrightarrow L^{2}\left(\mathbb{R}_{B}^{n}\right)$ is given by:

$$
\mathcal{F}_{B}\left(\delta_{\xi}\right)=e_{\xi}, \quad \text { with } \delta_{\xi}(\eta)=\delta_{\xi, \eta},
$$

where $\delta_{\xi, \eta}$ is the Kronecker symbol. We shall denote by $\mathcal{F}$ the usual Fourier transform on the abelian group $\mathbb{R}^{n}$ with its usual Lebesgue measure.

The action of $\mathbb{R}^{n}$ on $\mathbb{R}_{B}^{n}$ by translations yields a topological dynamical system whose naturally associated von Neumann algebra is the crossed product $L_{\infty}\left(\mathbb{R}_{B}^{n}\right) \times \mathbb{R}^{n}$. It is more convenient for applications to consider the commutant of this von Neumann algebra denoting it by $\mathcal{N}$. It is also a crossed product, namely the von Neumann algebra $L_{\infty}\left(\mathbb{R}^{n}\right) \times \mathbb{R}_{d}^{n}$. Then $\mathcal{N}$ is a type $I I_{\infty}$ factor with a faithful normal semifinite trace $\tau$. It can be described as the set of Borel essentially bounded families $\left(A_{\mu}\right)_{\mu \in \mathbb{R}_{B}^{n}}$ of bounded operators in $L^{2}\left(\mathbb{R}^{n}\right)$ which are $\mathbb{R}^{n}$ - equivariant, i.e. such that

$$
A_{\mu}=\sigma_{\mu}\left(A_{0}\right)=T_{-\mu} A_{0} T_{\mu}, \quad \forall \mu \in \mathbb{R}^{n} .
$$

Here and in the sequel we denote by $\sigma_{\mu}$ conjugation of any operator with the translation $T_{\mu}$ so that $\sigma_{\mu}(B)=T_{-\mu} B T_{\mu}$. If we denote by $M_{\varphi}$ the operator of multiplication by a bounded function $\varphi$, then examples of such families are given for any $\lambda$ by the families

$$
\left(\sigma_{\mu}\left(M_{e_{\lambda}}\right)\right)_{\mu \in \mathbb{R}_{B}^{n}}
$$

We choose the Fourier transform

$$
\mathcal{F} f(\zeta)=\frac{1}{(2 \pi)^{n / 2}} \int_{\mathbb{R}^{n}} e^{i x \zeta} f(x) d x .
$$

Then the von Neumann algebra $\mathcal{N}$ can be defined [27] as the completion in the Hilbert space $\mathcal{H}=L^{2}\left(\mathbb{R}^{n}\right) \otimes L^{2}\left(\mathbb{R}_{B}^{n}\right)$ of the set of operators $\left\{M_{e_{\lambda}} \otimes M_{e_{\lambda}}, T_{\lambda} \otimes 1\right\}$ when $\lambda$ ranges over $\mathbb{R}^{n}$.

There is a natural way to embed the $C^{*}$-algebra $\mathcal{A} P\left(\mathbb{R}^{n}\right)$ in $\mathcal{N}$ by setting

$$
\pi(f):=\left(\sigma_{\mu}\left(M_{f}\right)\right)_{\mu \in \mathbb{R}_{B}^{n}}
$$

This family then belongs to $\mathcal{N}$ and $\pi$ is clearly faithful. Viewed as an operator on $\mathcal{H}, \pi(f)$ is given by $\pi(f)(g)(x, \mu)=f(x+\mu) g(x, \mu)$. If $B=\left(B_{\mu}\right)_{\mu}$ is a positive element of $\mathcal{N}$, then we define the expectation $E(B)$ as the Haar integral:

$$
E(B):=\int_{\mathbb{R}_{B}^{n}} B_{\mu} d \alpha_{B}(\mu) .
$$

Since the family $B$ and $\alpha_{B}$ are translation invariant, the operator $E(B)$ clearly commutes with the translations in $L^{2}\left(\mathbb{R}^{n}\right)$ and is therefore given by a Fourier multiplier $\widetilde{M}\left(\varphi_{B}\right)$ with $\varphi_{B}$ a positive element of $L^{\infty}\left(\mathbb{R}^{n}\right)$. Recall that the Fourier multiplier $\widetilde{M}\left(\varphi_{B}\right)$ is conjugation of the multiplication operator $M_{\varphi}$ by the Fourier transform, i.e. $\widetilde{M}\left(\varphi_{B}\right)=\mathcal{F}^{-1} M_{\varphi} \mathcal{F}$. When the function $\varphi$ is in the Schwartz space, the operator $\widetilde{M}\left(\varphi_{B}\right)$ is convolution by the Schwartz 
function $\frac{1}{(2 \pi)^{n / 2}} \mathcal{F}^{-1} \varphi$. Hence the expectation $E$ takes values in the von Neumann algebra $\widetilde{M}\left(L^{\infty}\left(\mathbb{R}^{n}\right)\right)$, i.e.

$$
E: \mathcal{N} \longrightarrow \widetilde{M}\left(L^{\infty}\left(\mathbb{R}^{n}\right)\right)
$$

Now, using the usual Lebesgue integral on $\mathbb{R}^{n}$, and the normalisation of Coburn et al[27] we introduce the following definition of the trace $\tau$ :

$$
\tau(B)=\int_{\mathbb{R}^{n}} \varphi_{B}(\zeta) d \zeta
$$

Lemma 15.1. [27] The map $\tau$ is, up to constant, the unique positive normal faithful semifinite trace on $\mathcal{N}$.

Consider the trace on $\mathcal{N}$ evaluated on an operator of the form $M_{a} K$ where $a$ is almost periodic and $K$ is a convolution operator on $L^{2}\left(\mathbb{R}^{n}\right)$ arising from multiplication by an $L^{1}$ function $k$ on the Fourier transform. We have,

$$
\tau\left(M_{a} K\right)=\lim _{T \rightarrow+\infty} \frac{1}{(2 T)^{n}} \int_{(-T, T)^{n}} a(x) d x \int_{\mathbb{R}^{n}} k(\zeta) d \zeta .
$$

More generally, any pseudodifferential operator $A$ on $L^{2}\left(R^{n}, \mathbb{C}^{N}\right)$ with almost periodic coefficients of nonpositive order $m$ acting on $\mathbb{C}^{N}$-valued functions, can be viewed as a family over $\mathbb{R}_{B}^{n}$ of pseudodifferential operators on $\mathbb{R}^{n}$. To do this first take the symbol $a$ of $A$, then the operator $\sigma_{\mu}(A)$ is the pseudodifferential operator with almost periodic coefficients whose symbol is

$$
(x, \xi) \longmapsto a(x+\mu, \xi) .
$$

When $m \leqslant 0$, we obtain, in this way, an element of $\mathcal{N}$. We denote by $\Psi_{A P}^{0}$ the algebra of pseudodifferential operators with almost periodic coefficients and with non positive order. When the order $m$ of $A$ is $>0$ then the operator $A^{\sharp}$ given by the family $\left(\sigma_{\mu}(A)\right)_{\mu \in \mathbb{R}_{B}^{n}}$ is affiliated with $\mathcal{N}$. If the order $m$ of $A$ is $<-n$, then the bounded operator $A^{\sharp}$ is trace class with respect to the trace $\tau$ on the von Neumann algebra $\mathcal{N} \otimes M_{N}(\mathbb{C})[88$, Proposition 3.3] and we have:

$$
\tau\left(A^{\sharp}\right)=\lim _{T \rightarrow+\infty} \frac{1}{(2 T)^{n}} \int_{(-T,+T)^{n} \times \mathbb{R}^{n}} \operatorname{Tr}(a(x, \zeta)) d x d \zeta
$$

Indeed, the expectation $E\left(A^{\sharp}\right)$ is a pseudodifferential operator on $\mathbb{R}^{n}$ with symbol denoted by $E(a)$ and is independent of the $x$-variable, it is given by:

$$
E(a)(\zeta)=\lim _{T \rightarrow+\infty} \frac{1}{(2 T)^{n}} \int_{(-T,+T)^{n}} a(x, \zeta) d x .
$$

Hence the operator $E\left(A^{\sharp}\right)$ is precisely the Fourier multiplier $\widetilde{M}(E(a))$ and so:

$$
\tau\left(A^{\sharp}\right)=\int_{\mathbb{R}^{n}} \operatorname{Tr}(E(a)(\zeta)) d \zeta .
$$

Let $\Psi_{A P}^{\infty}$ be the space of one step polyhomogeneous classical pseudodifferential operators on $\mathbb{R}^{n}$ with almost periodic coefficients.

Theorem 15.2. Let $A$ be a (scalar) pseudodifferential operator with almost periodic coefficients on $\mathbb{R}^{n}$. We assume that the order $m$ of $A$ is $\leqslant-n$ and we denote by $a_{-n}$ the $-n$ homogeneous part of the symbol $a$. Then the operator $A^{\sharp}$ belongs to the Dixmier ideal 
$\mathcal{L}^{1, \infty}(\mathcal{N}, \tau)$. Moreover, the Dixmier trace $\tau_{\omega}\left(A^{\sharp}\right)$ of $A^{\sharp}$ associated with a limiting process $\omega$ does not depend on $\omega$ and is given by the formula:

$$
\tau_{\omega}\left(A^{\sharp}\right)=\frac{1}{n} \int_{\mathbb{R}_{B}^{n} \times \mathbb{S}^{n-1}} a_{-n}(x, \zeta) d \alpha_{B}(x) d \zeta .
$$

Proof. We denote as usual by $\Delta$ the Laplace operator on $\mathbb{R}^{n}$. The operator $A(1+\Delta)^{n / 2}$ is then a pseudodifferential operator with almost periodic coefficients and nonpositive order. Hence, the operator $\left[A(1+\Delta)^{n / 2}\right]^{\sharp}=A^{\sharp}\left(1+\Delta^{\sharp}\right)^{n / 2}$ belongs to the von Neumann algebra $\mathcal{N}$. Now the operator $\left(1+\Delta^{\sharp}\right)^{-n / 2}$ is a Fourier multiplier defined by the function $\zeta \mapsto\left(1+\zeta^{2}\right)^{-n / 2}$. Hence if, for $\lambda>0, E_{\lambda}$ is the spectral projection of the operator $(1+\Delta)^{-n / 2}$ corresponding to the interval $(0, \lambda)$ then the operator $1-E_{\lambda}$ is a Fourier multiplier as well. Let us suppose it is defined by the function $\zeta \mapsto f_{\lambda}\left(\left(\zeta^{2}+1\right)^{-n / 2}\right)$. It follows that the trace $\tau$ of the operator $1-E_{\lambda}$ is given by

$$
\int_{\mathbb{R}^{n}} f_{\lambda}\left(\frac{1}{\left(\zeta^{2}+1\right)^{n / 2}}\right) d \zeta
$$

It is easy to compute this integral and to show that it is proportional to $\frac{1}{\lambda}$. So the infimum of those $\lambda$ for which $\tau\left(1-E_{\lambda}\right) \leqslant t$ is precisely proportional to $\frac{1}{t}$. Hence the operator $\left(1+\Delta^{\sharp}\right)^{-n / 2}$, and hence $A$, belongs to the Dixmier ideal $\mathcal{L}^{1, \infty}(\mathcal{N}, \tau)$.

In order to compute the Dixmier trace of the operator $A$, we apply [89, Theorem 10.1] to deduce that the spectral $\tau$-density $N_{A}(\lambda)$ of $A$ has the asymptotic expansion

$$
N_{A}(\lambda)=\frac{\gamma_{0}(A)}{\lambda}(1+o(1)), \quad \lambda \rightarrow+\infty,
$$

where $\gamma_{0}(A)$ is given by:

$$
\gamma_{0}(A)=\frac{1}{n} \int_{\mathbb{R}_{B}^{n} \times \mathbb{S}^{n-1}} a_{-n}(x, \zeta) d \alpha_{B}(x) d \zeta
$$

Now, if $A$ is positive then by [10, Proposition 1]:

$$
\tau_{\omega}(A)=\lim _{\lambda \rightarrow+\infty} \lambda N_{A}(\lambda)=\gamma_{0}(A)
$$

This proves the theorem for positive $A$. Since the principal symbol map is a homomorphism, we deduce the result for general $A$.

The normalisation we have chosen for the trace in the von Neumann setting of this Section eliminates a factor of $\frac{1}{(2 \pi)^{n}}$ which occurs on the Wodzicki residue in the type $I$ theory.

15.2. Almost periodic spectral triple. We denote by $\mathcal{A}$ the $*$-subalgebra of $\mathcal{A} P^{\infty}\left(\mathbb{R}^{n}\right)$ of smooth almost periodic functions on $\mathbb{R}^{n}$. We take the Hilbert space on which the algebra $\mathcal{N}$ acts to be $B^{2}\left(\mathbb{R}^{n}\right) \otimes L^{2}\left(\mathbb{R}^{n}\right)$ where $B^{2}\left(\mathbb{R}^{n}\right)$ is the Hilbert space of almost periodic functions on $\mathbb{R}^{n}$ where the norm and inner product are given by the restriction of the Haar trace on $\mathcal{A} P^{\infty}\left(\mathbb{R}^{n}\right)$ to $\mathcal{A}$ (note that $\left.B^{2}\left(\mathbb{R}^{n}\right) \cong \ell^{2}\left(\mathbb{R}_{d}^{n}\right)\right)$. This type $I I_{\infty}$ von Neumann algebra is endowed with a faithful normal semifinite trace that we denote by $\tau$. (We note that the explicit formula for $\tau$ is as given in the previous subsection.)

The usual Dirac operator on $\mathbb{R}^{n}$ is denoted by $\mathcal{D}_{0}$. So, if $\mathcal{S}$ is the spin representation of $\mathbb{R}^{n}$ then $\mathcal{D}_{0}$ acts on smooth $\mathcal{S}$-valued functions on $\mathbb{R}^{n}$. The operator $\mathcal{D}_{0}$ is $\mathbf{Z}^{n}$-periodic and it 
is affiliated with the von Neumann algebra $\mathcal{N}_{\mathcal{S}}=\mathcal{N} \otimes \operatorname{End}(\mathcal{S})$. This latter is also a type $I I_{\infty}$ von Neumann algebra with the trace $\tau \otimes \operatorname{tr}$. More generally, for any $N \geqslant 1$, we shall denote by $\mathcal{N}_{\mathcal{S}, N}$ the von Neumann algebra $\mathcal{N} \otimes \operatorname{End}\left(\mathcal{S} \otimes \mathbb{C}^{N}\right)$ with the trace $\tau \otimes \operatorname{tr}$.

The algebra $\mathcal{A}$ and its closure are faithfully represented as $*$-subalgebras of the von Neumann algebra $\mathcal{N}_{\mathcal{S}}$. In the same way the algebra $\mathcal{A} \otimes M_{N}(\mathbb{C})$ can be viewed as a $*$-subalgebra of $\mathcal{N}_{\mathcal{S}, N}$. More precisely, if $a \in \mathcal{A}$ then the operator $a^{\sharp}$ defined by

$$
\left(a^{\sharp} f\right)(x, y):=a(x+y) f(x, y), \quad \forall f \in B^{2}\left(\mathbb{R}^{n}\right) \otimes L^{2}\left(\mathbb{R}^{n}\right),
$$

belongs to $\mathcal{N}_{S, N}$. The operator $a^{\sharp}$ is just the one associated with the zero-th order differential operator corresponding to multiplication by $a$. The same formula allows to represent $\mathcal{A}$ in $\mathcal{N}_{\mathcal{S}}$.

Proposition 15.3. The triple $\left(\mathcal{A}, \mathcal{N}_{\mathcal{S}}, \mathcal{D}_{0}^{\sharp}\right)$ is a semifinite spectral triple of finite dimension equal to $n$.

Proof. Note that the algebra $\mathcal{A}$ is unital. The differential operator $\mathcal{D}_{0}$ is known to be elliptic periodic and self-adjoint on $\mathbb{R}^{n}$. Therefore, the operator $\mathcal{D}_{0}^{\sharp}$ is affiliated with the von Neumann algebra $\mathcal{N}_{\mathcal{S}}$ and it is self-adjoint as a densely defined unbounded operator on the Hilbert space $B^{2}\left(\mathbb{R}^{n}\right) \otimes L^{2}\left(\mathbb{R}^{n}\right)$ with $\mathcal{D}_{0}^{2}=\Delta \otimes I d$ with $\Delta$ the usual Laplacian. For any smooth almost periodic function $f$ on $\mathbb{R}^{n}$, the commutator $\left[\mathcal{D}_{0}, f\right]$ is a 0 - th order almost periodic differential operator and so $\left[\mathcal{D}_{0}^{\sharp}, f\right]$ belongs to the von Neumann algebra $\mathcal{N}$.

On the other hand, the pseudodifferential operator $T=(\Delta+I)^{-1 / 2}$ is the Fourier multiplier associated with the function $k \mapsto \frac{1}{\left(\|k\|^{2}+1\right)^{1 / 2}}$. Therefore, its singular numbers $\mu_{t}(T)$ can be computed explicitly as in the proof of Theorem 15.2 and shown to be proportional to $t^{-1 / n}$.

\section{LESCH'S INDEX ThEOREM}

In this Section we describe (following [25]) a proof of an index theorem due to M. Lesch [70] (see also [82]) for Toeplitz operators with noncommutative symbol that relies on the zeta function approach to the Dixmier trace. We begin with a unital $C^{*}$-algebra $\mathcal{A}$ with a faithful finite trace, $\tau_{\mathcal{A}}$ satisfying $\tau_{\mathcal{A}}(1)=1$ and a continuous action $\alpha$ of $\mathbb{R}$ on $\mathcal{A}$ leaving $\tau_{\mathcal{A}}$ invariant.

We let $H_{\tau_{\mathcal{A}}}$ denote the Hilbert space completion of $\mathcal{A}$ in the inner product $(a \mid b)=\tau\left(b^{*} a\right)$. Then $\mathcal{A}$ is a Hilbert algebra and the left regular representation of $\mathcal{A}$ on itself extends by continuity to a representation, $a \mapsto \pi_{\tau_{\mathcal{A}}}(a)$ of $\mathcal{A}$ on $H_{\tau_{\mathcal{A}}}$ [42]. In what follows, we will drop the notation $\pi_{\tau_{\mathcal{A}}}$ and just denote the action of $\mathcal{A}$ on $H_{\tau_{\mathcal{A}}}$ by juxtaposition.

We now look at the induced representation, $\tilde{\pi}$, of the crossed product $C^{*}$-algebra $\mathcal{A} \times{ }_{\alpha} \mathbb{R}$ on $L^{2}\left(\mathbb{R}, H_{\tau_{\mathcal{A}}}\right)$. That is, $\tilde{\pi}$ is the representation $\pi \times \lambda$ obtained from the covariant pair, $(\pi, \lambda)$ of representations of the system $(\mathcal{A}, \mathbb{R}, \alpha)$ defined for $a \in \mathcal{A}, t, s \in \mathbb{R}$ and $\xi \in L^{2}\left(\mathbb{R}, H_{\tau_{\mathcal{A}}}\right)$ by:

$$
(\pi(a) \xi)(s)=\alpha_{s}^{-1}(a) \xi(s)
$$

and

$$
\lambda_{t}(\xi)(s)=\xi(s-t)
$$


Then, for a function $x \in L^{1}(\mathbb{R}, \mathcal{A}) \subset \mathcal{A} \times{ }_{\alpha} \mathbb{R}$, the action of $\tilde{\pi}(x)$ on a vector $\xi$ in $L^{2}\left(\mathbb{R}, H_{\tau_{\mathcal{A}}}\right)$ is defined as follows:

$$
(\tilde{\pi}(x) \xi)(s)=\int_{-\infty}^{\infty} \alpha_{s}^{-1}(x(t)) \xi(s-t) d t
$$

Now the twisted convolution algebra $L^{1}(\mathbb{R}, \mathcal{A}) \cap L^{2}\left(\mathbb{R}, H_{\tau}\right)$ is a dense subspace of $L^{2}\left(\mathbb{R}, H_{\tau}\right)$ and also a Hilbert algebra in the given inner product. As such, there is a canonical faithful, normal, semifinite trace, $\tau$, on the von Neumann algebra

$$
\mathcal{N}=\left(\tilde{\pi}\left(\mathcal{A} \times_{\alpha} \mathbb{R}\right)\right)^{\prime \prime}
$$

For functions $x, y: \mathbb{R} \rightarrow \mathcal{A} \subset H_{\tau_{\mathcal{A}}}$ which are in $L^{2}\left(\mathbb{R}, H_{\tau_{\mathcal{A}}}\right)$ and whose twisted left convolutions $\tilde{\pi}(x), \tilde{\pi}(y)$ define bounded operators on $L^{2}\left(\mathbb{R}, H_{\tau_{\mathcal{A}}}\right)$, this trace is given by:

$$
\tau\left(\tilde{\pi}(y)^{*} \tilde{\pi}(x)\right)=\langle x \mid y\rangle=\int_{-\infty}^{\infty} \tau_{\mathcal{A}}\left(x(t) y(t)^{*}\right) d t .
$$

In particular, if we identify $L^{2}(\mathbb{R})=L^{2}(\mathbb{R}) \otimes 1_{\mathcal{A}} \subset L^{2}\left(\mathbb{R}, H_{\tau_{\mathcal{A}}}\right)$ then any scalar-valued function $x$ on $\mathbb{R}$ which is the Fourier transform $x=\widehat{f}$ of a bounded $L^{2}$ function, $f$ will have the properties that $x \in L^{2}\left(\mathbb{R}, H_{\tau_{\mathcal{A}}}\right)$ and $\tilde{\pi}(x)$ is a bounded operator. For such scalar functions $x$, the operator $\tilde{\pi}(x)$ is just the usual convolution by the function $x$ and is usually denoted by $\lambda(x)$ since it is just the integrated form of $\lambda$. The next Lemma follows easily from these considerations.

Lemma 16.1. With the hypotheses and notation discussed above

(i) if $h \in L^{2}(\mathbb{R})$ with $\lambda(h)$ bounded and $a \in \mathcal{A}$, then defining $f: \mathbb{R} \rightarrow H_{\tau}$ via $f(t)=a h(t)$ we see that $f \in L^{2}\left(\mathbb{R}, H_{\tau_{\mathcal{A}}}\right)$ and $\tilde{\pi}(f)=\pi(a) \lambda(h)$ is bounded,

(ii) if $g \in L^{1}(\mathbb{R}) \cap L^{\infty}(\mathbb{R})$ and $a \in \mathcal{A}$ then $\pi(a) \lambda(\hat{g})$ is trace-class in $\mathcal{N}$ and

$$
\operatorname{Tr}(\pi(a) \lambda(\hat{g}))=\tau_{\mathcal{A}}(a) \int_{-\infty}^{\infty} g(t) d t .
$$

Proof. To see part (i), let $\xi \in C_{c}\left(\mathbb{R}, H_{\tau_{\mathcal{A}}}\right) \subseteq L^{2}\left(\mathbb{R}, H_{\tau_{\mathcal{A}}}\right)$. Then

$$
\begin{aligned}
(\tilde{\pi}(f) \xi)(s) & =\int_{-\infty}^{\infty} \alpha_{s}^{-1}(f(t)) \xi(s-t) d t \\
& =\int_{-\infty}^{\infty} \alpha_{s}^{-1}(a) h(t) \xi(s-t) d t \\
& =\alpha_{s}^{-1}(a) \int_{-\infty}^{\infty} h(t) \xi(s-t) d t \\
& =\alpha_{s}^{-1}(a)(\lambda(h) \xi)(s) \\
& =(\pi(a) \lambda(h) \xi)(s) .
\end{aligned}
$$

To see part (ii) we can assume that $g$ is nonnegative and $a$ is self-adjoint. Then let $g=$ $g^{1 / 2} g^{1 / 2}$ so that $g^{1 / 2} \in L^{2} \cap L^{\infty}$ and so $\lambda\left(\widehat{g^{1 / 2}}\right)$ is bounded. Now,

$$
\pi(a) \lambda(\widehat{g})=\pi(a) \lambda\left(\widehat{g^{1 / 2}}\right) \pi\left(1_{\mathcal{A}}\right) \lambda\left(\widehat{g^{1 / 2}}\right) .
$$


Then, $\pi(a) \lambda\left(\widehat{g^{1 / 2}}\right)=\tilde{\pi}(x)$ where $x(t)=a \widehat{g^{1 / 2}}(t)$ and $\pi\left(1_{\mathcal{A}}\right) \lambda\left(\widehat{g^{1 / 2}}\right)=\tilde{\pi}(y)$ where $y(t)=$ $1_{\mathcal{A}} \widehat{g^{1 / 2}}(t)$. So, $\tilde{\pi}(x)$ and $\tilde{\pi}(y)$ are in $\mathcal{N}_{s a}$ and $\pi(a) \lambda(\widehat{g})=\tilde{\pi}(x) \tilde{\pi}(y)$.

Hence,

$$
\begin{aligned}
\tau(\pi(a) \lambda(\widehat{g})) & =\tau(\tilde{\pi}(x) \tilde{\pi}(y)) \\
& =\int_{-\infty}^{\infty} \tau(x(t) y(t)) d t \\
& =\tau_{\mathcal{A}}(a) \int_{-\infty}^{\infty}\left|\widehat{g^{1 / 2}}(t)\right|^{2} d t=\tau_{\mathcal{A}}(a) \int_{-\infty}^{\infty} g(s) d s
\end{aligned}
$$

By construction $\mathcal{N}$ is a semifinite von Neumann algebra with faithful, normal, semifinite trace, $\tau$, and a faithful representation $\pi: \mathcal{A} \rightarrow \mathcal{N}$ [42]. For each $t \in \mathbb{R}, \lambda_{t}$ is a unitary in $U(\mathcal{N})$. In fact the one-parameter unitary group $\left\{\lambda_{t} \mid t \in \mathbb{R}\right\}$ can be written $\lambda_{t}=e^{i t \mathcal{D}}$ where $\mathcal{D}$ is the unbounded self-adjoint operator

$$
\mathcal{D}=\frac{1}{2 \pi i} \frac{d}{d s}
$$

which is affiliated with $\mathcal{N}$. In the Fourier transform picture (i.e., the spectral picture for $\mathcal{D}$ ) of the previous proposition, $\mathcal{D}$ becomes multiplication by the independent variable and so $f(\mathcal{D})$ becomes pointwise multiplication by the function $f$. That is,

$$
\tilde{\pi}(\hat{f})=\lambda(\hat{f})=f(\mathcal{D}) .
$$

And, hence, if $f$ is a bounded $L^{1}$ function, then:

$$
\tau(f(\mathcal{D}))=\int_{-\infty}^{\infty} f(t) d t
$$

By this discussion and the previous lemma, we have the following result

Lemma 16.2. If $f \in L^{1}(\mathbb{R}) \cap L^{\infty}(\mathbb{R})$ and $a \in \mathcal{A}$ then $\pi(a) f(\mathcal{D})$ is trace-class in $\mathcal{N}$ and

$$
\tau(\pi(a) f(\mathcal{D}))=\tau(a) \int_{-\infty}^{\infty} f(t) d t
$$

We let $\delta$ be the densely defined (unbounded) $*$-derivation on $\mathcal{A}$ which is the infinitesimal generator of the representation $\alpha: \mathbb{R} \rightarrow A u t(\mathcal{A})$ and let $\hat{\delta}$ be the unbounded $*$-derivation on $\mathcal{N}$ which is the infinitesimal generator of the representation $A d \circ \lambda: \mathbb{R} \rightarrow \operatorname{Aut}(\mathcal{N})$ (here $\operatorname{Ad}\left(\lambda_{t}\right)$ denotes conjugation by $\left.\lambda_{t}^{*} \cdot \lambda_{t}\right)$. Now if $a \in \operatorname{dom}(\delta)$ then clearly $\pi(a) \in \operatorname{dom}(\hat{\delta})$ and $\pi(\delta(a))=\hat{\delta}(\pi(a))$. By [12] Proposition 3.2.55 (and its proof) we have that $\pi(\delta(a))$ leaves the domain of $\mathcal{D}$ invariant and

$$
\pi(\delta(a))=2 \pi i[\mathcal{D}, \pi(a)]
$$

We are now in a position to state and prove Lesch's index theorem.

Theorem $16.3([25])$. Let $\tau_{\mathcal{A}}$ be a faithful finite trace on the unital $C^{*}$-algebra, $\mathcal{A}$, which is invariant for an action $\alpha$ of $\mathbb{R}$. Let $\mathcal{N}$ be the semifinite von Neumann algebra $\left(\tilde{\pi}\left(\mathcal{A} \times{ }_{\alpha} \mathbb{R}\right)\right)^{\prime \prime}$, and let $\mathcal{D}$ be the infinitesimal generator of the canonical representation $\lambda$ of $\mathbb{R}$ in $U(\mathcal{N})$. Then, the representation $\pi: \mathcal{A} \rightarrow \mathcal{N}$ defines a $\mathcal{L}^{(1, \infty)}$ summable Breuer-Fredholm module 
$(\mathcal{N}, \mathcal{D})$ for $\mathcal{A}$. Moreover, if $P$ is the nonnegative spectral projection for $\mathcal{D}$ and $u \in U(\mathcal{A})$ is also in the domain of $\delta$, then $T_{u}:=P \pi(u) P$ is Breuer-Fredholm in $P \mathcal{N} P$ and

$$
\operatorname{ind}\left(T_{u}\right)=\frac{1}{2 \pi i} \tau\left(u \delta\left(u^{*}\right)\right)
$$

Proof. It is easy to see that $\mathcal{D}$ satisfies $\left(1+\mathcal{D}^{2}\right)^{-1 / 2} \in \mathcal{L}^{(1, \infty)}$. By the previous discussion, for any $a \in \operatorname{dom}(\delta)$ we have $\pi(\delta(a))=2 \pi i[\mathcal{D}, \pi(a)]$. Since the domain of $\delta$ is dense in $\mathcal{A}$ we see that $\pi$ defines a $\mathcal{L}^{(1, \infty)}$ summable Breuer-Fredholm module for $\mathcal{A}$.

By Theorem 13.2,

$$
\operatorname{ind}\left(T_{u}\right)=\lim _{p \rightarrow 1^{+}} \frac{1}{2}(p-1) \operatorname{Tr}\left(\pi(u)\left[\mathcal{D}, \pi\left(u^{*}\right)\right]\left(1+\mathcal{D}^{2}\right)^{-p / 2}\right)
$$

and hence

$$
\begin{aligned}
\operatorname{ind}\left(T_{u}\right) & =\lim _{p \rightarrow 1^{+}} \frac{1}{2}(p-1) \frac{1}{2 \pi i} \operatorname{Tr}\left(\pi\left(u \delta\left(u^{*}\right)\right)\left(1+\mathcal{D}^{2}\right)^{-p / 2}\right) \\
& =\lim _{p \rightarrow 1^{+}} \frac{1}{2}(p-1) \frac{1}{2 \pi i} \tau\left(u \delta\left(u^{*}\right)\right) \int_{-\infty}^{\infty}\left(1+t^{2}\right)^{-p / 2} d t \\
& =\lim _{p \rightarrow 1^{+}} \frac{1}{2 \pi i} \tau\left(u \delta\left(u^{*}\right)\right) \frac{1}{2}(p-1) C_{p / 2}=\frac{1}{2 \pi i} \tau\left(u \delta\left(u^{*}\right)\right)
\end{aligned}
$$

\section{The Hochschild Class of the Chern Character}

The theorem we discuss in this Section, for $\mathcal{N}=\mathcal{L}(\mathcal{H})$ and $1<p<\infty$ ( $p$ integral), was proved in lectures by Alain Connes at the Collège de France in 1990. A version of this argument appeared in [57]. The extension of this argument to general semifinite von Neumann algebras, with the additional hypothesis that the unbounded self-adjoint operator $\mathcal{D}$ (which will form part of a spectral triple) have bounded inverse, is presented by Benameur and Fack, [10]. A simpler strategy using the pseudodifferential calculus of Connes-Moscovici, [40], was communicated to us by Nigel Higson. In conjunction with the results in [25], Higson's argument appears to generalise to the semifinite case, however, we will not describe the details here focusing instead on another approach.

In [21] the theorems of Connes and Benameur-Fack were extended. First a proof was given for the case $p=1$ and second the hypothesis, in the type $I I_{\infty}$ case, that $\mathcal{D}$ has bounded inverse was removed. This is crucial due to the 'zero-in-the-spectrum' phenomenon for $\mathcal{D}$. That is, for type $I I \mathcal{N}$, zero is generically in the point and/or continuous spectrum, [54] and hence we are not dealing with just the simple problem that arises in the type $I$ case posed by a finite dimensional kernel. One feature of the approach in [21] is that the strategy of the proof is the same for all $p \geqslant 1$, and is independent of the type of the von Neumann algebra $\mathcal{N}$.

We explain the general semifinite version of the type $I$ result in [31, IV.2. $\gamma$ ] which identifies the Hochschild class of the Chern character of a $(p, \infty)$-summable spectral triple. 
Theorem 17.1. Let $(\mathcal{A}, \mathcal{H}, \mathcal{D})$ be a $Q C^{k}(p, \infty)$-summable spectral triple with $p \geqslant 1$ integral and $k=\max \{2, p-2\}$. Then

1) A Hochschild cocycle on $\mathcal{A}$ is defined by

$$
\varphi_{\omega}\left(a_{0}, \ldots, a_{p}\right)=\lambda_{p} \tau_{\omega}\left(\Gamma a_{0}\left[\mathcal{D}, a_{1}\right] \cdots\left[\mathcal{D}, a_{p}\right]\left(1+\mathcal{D}^{2}\right)^{-p / 2}\right)
$$

2) For all Hochschild $p$-cycles $c \in C_{p}(\mathcal{A})$ (i.e., $b c=0$ ),

$$
\left\langle\varphi_{\omega}, c\right\rangle=\left\langle C h_{F_{\mathcal{D}}}, c\right\rangle
$$

where $C h_{F_{\mathcal{D}}}$ is the Chern character in cyclic cohomology of the pre-Fredholm module over $\mathcal{A}$ with $F_{\mathcal{D}}=\mathcal{D}\left(1+\mathcal{D}^{2}\right)^{-1 / 2}$.

Remark 17.2. Here $\tau_{\omega}$ is the Dixmier trace associated to any state $\omega \in D\left(\mathbb{R}_{+}^{*}\right)$.

The two most important corollaries of Theorem 17.1 are the following.

Corollary 17.3. Let $(\mathcal{A}, \mathcal{H}, \mathcal{D})$ be as in Theorem 17.1. If $c=\sum_{i} a_{0}^{i} \otimes a_{1}^{i} \otimes \cdots \otimes a_{p}^{i}$ is a Hochschild $p$-cycle, then

$$
\Gamma \sum_{i} a_{0}^{i}\left[\mathcal{D}, a_{1}^{i}\right] \cdots\left[\mathcal{D}, a_{p}^{i}\right]\left(1+\mathcal{D}^{2}\right)^{-p / 2}
$$

is (Dixmier)-measurable.

This corollary is relevant to the axioms of noncommutative spin geometry since it tells us that when we use the noncommutative integration theory provided by the Dixmier trace we do not need to worry which functional $\omega$ we use to define the integration when we apply it to Hochschild cycles.

Corollary 17.4. With $(\mathcal{A}, \mathcal{H}, \mathcal{D})$ as in Theorem 17.1, and supposing that $C h_{F_{\mathcal{D}}}$ pairs nontrivially with $H_{p}(\mathcal{A})$, then

$$
\tau_{\omega}\left(\left(1+\mathcal{D}^{2}\right)^{-p / 2}\right) \neq 0
$$

Remark 17.5. The hypothesis of the Corollary is that there exists some Hochschild $p$-cycle such that $\left\langle I C h_{F_{\mathcal{D}}}, c\right\rangle \neq 0$ (where $I$ is the map defined by Connes, [31] subsection III.1. $\gamma$ pp 199-200). Computing this pairing using Theorem 17.1 above, we see that $\left(1+\mathcal{D}^{2}\right)^{-p / 2}$ can not have zero Dixmier trace for any choice of Dixmier functional $\omega$. For if $\left(1+\mathcal{D}^{2}\right)^{-p / 2}$ did have vanishing Dixmier trace, and $c=\sum_{i} a_{0}^{i} \otimes \cdots \otimes a_{p}^{i}$ is any Hochschild cycle

$$
\begin{aligned}
\left|\left\langle I C h_{F_{\mathcal{D}}}, c\right\rangle\right| & =\left|\sum_{i} \tau_{\omega}\left(\Gamma a_{0}^{i}\left[\mathcal{D}, a_{1}^{i}\right] \cdots\left[\mathcal{D}, a_{p}^{i}\right]\left(1+\mathcal{D}^{2}\right)^{-p / 2}\right)\right| \\
& \leqslant \sum_{i}\left\|\Gamma a_{0}^{i}\left[\mathcal{D}, a_{1}^{i}\right] \cdots\left[\mathcal{D}, a_{p}^{i}\right]\right\| \tau_{\omega}\left(\left(1+\mathcal{D}^{2}\right)^{-p / 2}\right)=0 .
\end{aligned}
$$

Hence if the pairing is nontrivial, the Dixmier trace can not vanish on $\left(1+\mathcal{D}^{2}\right)^{-p / 2}$.

There are subtle points in the proof. One first assumes that the triple triple $(\mathcal{A}, \mathcal{H}, \mathcal{D})$ has $\mathcal{D}$ invertible (by replacing $(\mathcal{A}, \mathcal{H}, \mathcal{D})$ by $\left(\mathcal{A}, \mathcal{H}^{2}, \mathcal{D}_{m}\right)$ with $\mathcal{D}_{m}=\left(\begin{array}{cc}\mathcal{D} & m \\ m & -\mathcal{D}\end{array}\right)$ if necessary). Then one needs to verify that $\varphi_{\omega}$ in Theorem 17.1 does not depend on this replacement. One 
also requires the verification that the functional $\varphi_{\omega}$ is indeed a Hochschild cocycle but this quite simple.

Lemma 17.6. Let $p \geqslant 1$ and suppose that $(\mathcal{A}, \mathcal{H}, \mathcal{D})$ is a $Q C^{1}(p, \infty)$-summable spectral triple. Then the multilinear functional

$$
\varphi_{\omega}\left(a_{0}, \ldots, a_{p}\right)=\lambda_{p} \tau_{\omega}\left(\Gamma a_{0}\left[\mathcal{D}, a_{1}\right] \cdots\left[\mathcal{D}, a_{p}\right]\left(1+\mathcal{D}^{2}\right)^{-p / 2}\right)
$$

is a Hochschild cocycle.

Proof. By Lemma 3 of [21] and the trace property of the Dixmier trace, we have

$$
\begin{aligned}
\left(b \varphi_{\omega}\right)\left(a_{0}, \ldots, a_{p}\right)= & (-1)^{p-1} \lambda_{p} \tau_{\omega}\left(\Gamma a_{0}\left[\mathcal{D}, a_{1}\right] \cdots\left[\mathcal{D}, a_{p-1}\right] a_{p}\left(1+\mathcal{D}^{2}\right)^{-p / 2}\right) \\
& -(-1)^{p-1} \lambda_{p} \tau_{\omega}\left(\Gamma a_{0}\left[\mathcal{D}, a_{1}\right] \cdots\left[\mathcal{D}, a_{p-1}\right]\left(1+\mathcal{D}^{2}\right)^{-p / 2} a_{p}\right) .
\end{aligned}
$$

As $(\mathcal{A}, \mathcal{H}, \mathcal{D})$ is $Q C^{1}$,

$$
\left[\left(1+\mathcal{D}^{2}\right)^{-p / 2}, a_{p}\right]=-\sum_{k=0}^{p-1}\left(1+\mathcal{D}^{2}\right)^{-(p-k) / 2}\left[\left(1+\mathcal{D}^{2}\right)^{1 / 2}, a_{p}\right]\left(1+\mathcal{D}^{2}\right)^{-(1+k) / 2},
$$

and this is trace class. So $a_{p}\left(1+\mathcal{D}^{2}\right)^{-p / 2}=\left(1+\mathcal{D}^{2}\right)^{-p / 2} a_{p}$ modulo trace class operators, and so the two terms above cancel.

\section{LiDSKII TYPE FORMUlA FOR DiXMiER TRACES}

A semifinite analogue of the classical Lidskii theorem stated in terms of the (so-called) Brown spectral measure $\mu_{T}$ of $T \in \mathcal{N}$ asserts [16] that

$$
\tau(T)=\int_{\sigma(T) \backslash\{0\}} \lambda d \mu_{T}(\lambda) .
$$

In the case, when $\mathcal{N}=\mathcal{L}(\mathcal{H})$ and $\tau$ is the standard trace $\operatorname{Tr}$ the equality above reduces to the classical case asserting that the trace $\operatorname{Tr}(T)$ of an arbitrary trace class operator $T$ is given by the sum $\sum_{n \geqslant 1} \lambda(T)$, where $\left\{\lambda_{n}(T)\right\}_{n \geqslant 1}$ is the sequence of eigenvalues of $T$, arranged in decreasing order of absolute values of $\lambda_{n}$ and counting multiplicities. Note, that in the case $T \geqslant$ 0 , the equality $\operatorname{Tr}(T)=\sum_{n \geqslant 1} \lambda_{n}(T)$ follows immediately from the spectral theorem for compact operators. If $T^{*}=T$, then again, by the spectral theorem we can select the orthonormal basis of $\mathcal{H}$ consisting of eigenvalues of $T$ and still infer Lidskii's theorem without any difficulty. Here it is worth observing that the assumption $T^{*}=T$ belongs to the ideal $\mathcal{L}^{1}(\mathcal{H})$ of all trace class operators on $\mathcal{H}$ implies the absolute convergence of the series $\sum_{n \geqslant 1}\left|\lambda_{n}(T)\right|$. The latter fact guarantees the convergence of the series $\sum_{n \geqslant 1} \lambda_{n}(T)$ in whatever ordering of the set of all eigenvalues for $T$ is chosen (in particular, for the decreasing ordering of absolute values of $T$ ).

The core difference of this situation with the setting of Dixmier traces consists in the fact that the series $\sum_{n \geqslant 1}\left|\lambda_{n}(T)\right|$ diverges for every normal $T \in \mathcal{L}^{(1, \infty)}(\mathcal{H}) \backslash \mathcal{L}^{1}(\mathcal{H})$. Therefore, even though for a given $T=T^{*} \in \mathcal{L}^{(1, \infty)}(\mathcal{H})$ we define $\tau_{\omega}(T)$ as the difference $\tau_{\omega}\left(T_{+}\right)-\tau_{\omega}\left(T_{-}\right)$ 
where each number is computed according to the definition $\omega-\lim _{N \rightarrow \infty} \frac{1}{\log (1+N)} \sum_{n=1}^{N} \lambda_{n}\left(T_{ \pm}\right)$, it is by no means clear that we have

$$
\tau_{\omega}(T)=\omega-\lim _{N \rightarrow \infty} \frac{1}{\log (1+N)} \sum_{n=1}^{N} \lambda_{n}(T)
$$

for the special enumeration of the set $\left\{\lambda_{n}(T)\right\}_{n \geqslant 1}$ given by the decreasing order of absolute values of $\left|\lambda_{n}(T)\right|$; or for that matter for any enumeration of this set. This difficulty becomes even more pronounced in the case of a general semifinite von Neumann albegra.

Note that the restriction $\mu_{t}(T) \leqslant \frac{C}{t}, t \geqslant 1$ imposed on $T \in \mathcal{L}^{(1, \infty)}(\mathcal{N}, \tau)$ in the theorem below, implies that $T$ belongs to the ideal $N\left(\psi_{1}\right)(\mathcal{N}, \tau)$ (see the definition of $\psi_{1}$ in Section 2.1 and the definition of r.i. ideal $N(\psi)$ in Section 2.2). Everywhere below, we assume that $\omega \in C D\left(\mathbb{R}_{+}^{*}\right.$ ) (see Section 5.2). When we apply $\omega$ to a sequence $x \in \ell_{\infty}$, we identify $x$ with its image $i(x)$ in $L_{\infty}\left(\mathbb{R}_{+}\right)$(see Remark 5.3).

Theorem 18.1 ([8]). (i). If $(\mathcal{N}, \tau)$ is a semifinite von Numann albegra and $T \in$ $\mathcal{L}^{(1, \infty)}(\mathcal{N}, \tau)$ satisfies $\mu_{t}(T) \leqslant \frac{C}{t}, t \geqslant 1$ for some $C>0$, then

$$
\tau_{\omega}(T)=\omega-\lim _{t \rightarrow \infty} \frac{1}{\log (1+t)} \int_{\lambda \notin \frac{1}{t} G} \lambda d \mu_{T}(\lambda) .
$$

(ii). Let $T$ be a compact operator on a Hilbert space $\mathcal{H}$, such that $\mu_{n}(T) \leqslant \frac{C}{n}, n \geqslant 1$ for some $C>0$. Let $\lambda_{1}, \lambda_{2}, \ldots$ be the list of eigenvalues of the operator $T$ counted with multiplicities such that $\left|\lambda_{1}\right| \geqslant\left|\lambda_{2}\right| \geqslant \ldots$. Then

$$
\tau_{\omega}(T)=\omega-\lim _{t \rightarrow \infty} \frac{1}{\log (1+t)} \sum_{\lambda \in \sigma(T), \lambda \notin \frac{1}{t} G} \lambda \mu_{T}(\lambda)=\omega-\lim _{N \rightarrow \infty} \frac{1}{\log (1+N)} \sum_{i=1}^{N} \lambda_{i},
$$

where $\mu_{T}(\lambda)$ is the algebraic multiplicity of the eigenvalue $\lambda$ and $G$ is an arbitrary bounded neighborhood of $0 \in \mathbb{C}$.

Here, we emphasize the fact that $G$ is an arbitrary bounded neighborhood of 0 . In the case $\mathcal{N}=\mathcal{L}(\mathcal{H})$, formula $(25)$ says that we need to compute the sum of all eigenvalues $\lambda_{j}(T)$ which do not belong to the "squeezed" neighborhood $\frac{1}{t} G$ as $t \rightarrow \infty$ and then to apply $\omega$-limit. It is interesting to emphasize the special case of formula (25) for measurable operators belonging to the ideal $N\left(\psi_{1}\right)$. The result below should be compared with the results of Theorems 6.6 and 6.7 .

Corollary 18.2. If $T$ is a (Connes-Dixmier) measurable operator satisfying the assumption of Theorem 18.1 (i) (respectively, (ii)), then

$$
\tau_{\omega}(T)=\lim _{t \rightarrow \infty} \frac{1}{\log (1+t)} \int_{\lambda \notin \frac{1}{t} G} \lambda d \mu_{T}(\lambda) \quad\left(\text { resp. }=\lim _{N \rightarrow \infty} \frac{1}{\log (1+N)} \sum_{i=1}^{N} \lambda_{i}\right),
$$

for every $\omega \in C D\left(\mathbb{R}_{+}^{*}\right)$. 
Fix an orthonormal basis in $\mathcal{H}$ and identify every element $x \in \mathcal{L}(\mathcal{H})$ with its matrix $\left(x_{i j}\right)_{i, j=1}^{\infty}$. It is well-known [56] that the triangular truncation operator $\mathfrak{T}$ given by

$$
\mathfrak{T}(x)=\left\{\begin{array}{cc}
x_{i j}, & i \geqslant j \\
0, & i<j
\end{array}\right.
$$

acts boundedly from the trace class $\mathcal{L}^{1}(\mathcal{H})$ into $\mathcal{L}^{(1, \infty)}(\mathcal{H})$. Noting that $\mathfrak{T}(x)-\operatorname{diag}(x)$ is quasinilpotent for every $x \in \mathcal{L}^{1}(\mathcal{H})$, we obtain the following

Corollary 18.3. The operator $\mathfrak{T}(x)$ is Connes-Dixmier measurable for every $x \in \mathcal{L}^{1}(\mathcal{H})$, moreover $\tau_{\omega}(\mathfrak{T}(x))=0, \omega \in C D\left(\mathbb{R}_{+}^{*}\right)$.

We conclude with the following result immediately following from Theorem 18.1 (see also [52, Proposition 1]).

Corollary 18.4. Let $M$ be a compact $n$-dimensional Riemannian manifold and let $T$ be a pseudodifferential operator of order $-n$ on $M$. Then

$$
\tau_{\omega}(T)=\lim _{N \rightarrow \infty} \frac{1}{\log N} \sum_{k=1}^{N} \lambda_{k} .
$$

\section{REFERENCES}

[1] M. Adler, On a trace functional for formal pseudodifferential operators and the symplectic structure of the Korteweg de Vries type equations, Inv. Math. 50 (1979), 219-248.

[2] S. Albeverio, D. Guido, A. Ponosov, S. Scarlatti, Singular traces and compact operators, J. Funct. Anal. 137 (1996), 281-302.

[3] S. Albeverio, D. Guido, A. Ponosov, S. Scarlatti, Singular traces and nonstandard analysis, Advances in analysis, probability and mathematical physics (Blaubeuren, 1992), 3-19, Math. Appl., 314, 1995.

[4] S. Albeverio, D. Guido, A. Ponosov, S. Scarlatti, Nonstandard representation of nonnormal traces. Dynamics of complex and irregular systems (Bielefeld, 1991), 1-11, Bielefeld Encount. Math. Phys., VIII, 1993.

[5] M.F. Atiyah, V. Patodi, I. M. Singer, Spectral Asymmetry and Riemannian Geometry, I, Proc. Camb. Phil. Soc., 77 (1975), 43-69.

[6] M.F. Atiyah, V. Patodi, I. M. Singer, Spectral Asymmetry and Riemannian Geometry. III, Proc. Camb. Phil. Soc., 79 (1976), 71-99.

[7] M.F. Atiyah, Elliptic operators, discrete groups and von Neumann algebras, Colloque "Analyse et Topologie" en l'Honneur de Henri Cartan (Orsay, 1974), pp. 43-72. Asterisque, No. 32-33, Soc. Math. France, Paris, 1976.

[8] N. Azamov, F. Sukochev, A Lidskii type formula for Dixmier traces, C.R. Acad. Sci. Paris, Ser. I, 340 (2005), 107-112.

[9] M.T. Benameur, A.L. Carey, J. Phillips, A. Rennie, F.A. Sukochev, K.P. Wojciechowski, An analytic approach to spectral flow in von Neumann algebras, Analysis, Geometry and Topology of Elliptic Operators. Papers in Honor of Krzysztof P Wojciechowski, 297-352, World Sci. Publ., Singapore, 2006 .

[10] M-T. Benameur, T. Fack, Type II Noncommutative Geometry. I. Dixmier Trace in von Neumann Algebras, Adv. Math. 199 (2006), 29-87.

[11] B. Boo $\beta$-Bavnbek, M. Lesch, J. Phillips, Unbounded Fredholm Operators and Spectral Flow, Can. J. Math. 57 (2005) 225-250

[12] O. Bratteli, D. Robinson, Operator Algebras and Quantum Statistical Mechanics I, Springer-Verlag, New York-Heidelberg-Berlin, 1979.

[13] M.S. Braverman, A.A. Mekler, The Hardy-Littlewood property for symmetric spaces, (Russian) Sibirsk. Mat. Z. 18 (1977), no. 3, 522-540.

[14] M. Breuer, Fredholm Theories in von Neumann algebras. I, Math. Ann., 178 (1968), 243-254. 
[15] M. Breuer, Fredholm Theories in von Neumann algebras. II, Math. Ann., 180 (1969), 313-325.

[16] L.G. Brown, Lidskii theorem in the type II case, Geometric methods in operator algebras (Kyoto, 1983), 1-35, Pitman Res. Notes Math. Ser., 123, Longman Sci. Tech., Harlow, 1986.

[17] V.I. Chilin, F.A. Sukochev, Symmetric spaces over semifinite von Neumann algebras. (Russian) Dokl. Akad. Nauk SSSR 313 (1990), no. 4, 811-815; translation in Soviet Math. Dokl. 42 (1991), no. 1, 97-101.

[18] V.I. Chilin, F.A. Sukochev, Weak convergence in non-commutative symmetric spaces, J. Operator Theory 31 (1994), 35-65.

[19] A.L. Carey, J. Phillips, Unbounded Fredholm Modules and Spectral Flow, Canadian J. Math., 50 (1998), no. 4, 673-718.

[20] A.L. Carey, J. Phillips, Spectral flow in $\Theta$-summable Fredholm modules, eta invariants and the JLO cocycle, K Theory 31 (2004), 135-194.

[21] A.L. Carey, J. Phillips, A. Rennie, F. A. Sukochev, The Hochschild Class of the Chern Character of Semifinite Spectral Triples, J. Funct. Analysis 213 (2004), 111-153.

[22] A.L. Carey, J. Phillips, A. Rennie, F. Sukochev, The local index theorem in semifinite von Neumann algebras I. Spectral Flow, Advances in Math to appear.

[23] A.L. Carey, J. Phillips, A. Rennie, F. Sukochev, The Local Index Theorem in semifinite von Neumann algebras II: the even case, Advances in Math to appear.

[24] A.L. Carey, J. Phillips, F. A. Sukochev, On unbounded p-summable Fredholm modules, Advances in Math. 151 (2000), 140-163.

[25] A.L. Carey, J. Phillips, F. A. Sukochev, Spectral Flow and Dixmier Traces, Advances in Math. 173 (2003), 68-113.

[26] F. Cipriani, E. Guido, S. Scarlatti, A remark on trace properties of $K$-cycles, J. Operator Theory 35 (1996), 179-189.

[27] L.A. Coburn, R.G. Douglas, D.G. Schaeffer, I.M. Singer, $C^{*}$-algebras of Operators on a Half Space II Index Theory, IHES Publ. Math. 40 (1971), 69-80.

[28] A. Connes, Noncommutative Differential Geometry, Publ. Math. Inst. Hautes Etudes Sci., 62 (1985), 41-44.

[29] A. Connes, Cyclic Cohomology of Banach Algebras and Characters of $\theta$-summable Fredholm Modules, $K$-Theory 1 (1988), 519-548.

[30] A. Connes, Compact Metric Spaces, Fredholm Modules and Hyperfiniteness, Ergodic Theory and Dynamical Systems 9 (1989), 207-220.

[31] A. Connes, Noncommutative Geometry, Acad. Press, San Diego, 1994.

[32] A. Connes, Geometry From the Spectral Point of View, Lett. Math. Phys., 34 (1995), 203-238.

[33] A. Connes, The action functional in noncommutative geometry, Commun. Math. Phys. 117 (1988), 673-683.

[34] A. Connes, Essay on Physics and Noncommutative Geometry, The interface of mathematics and particle physics, Oxford Univ. Press, New York, 1990, 9-48.

[35] A. Connes, Noncommutative geometry and reality J. Math. Phys. 36 (1995), 6194-6231.

[36] A. Connes, Sur la théorie non commutative de l'integration Algèbres d'opérateurs, Lecture Notes in Math., Springer-Verlag 725 (1979), 19-143.

[37] A. Connes, Gravity coupled to matter and the foundations of noncommutative geometry, Commun. Math. Phys. 182 (1996), 155-176.

[38] A. Connes, Traces de Dixmier, modules de Fredholm et geometrie riemannienne, in Conformal field theories and related topics (Annecy-le-Vieux, 1988). Nuclear Phys. In Proc. Suppl. 5b (1988), 65-70.

[39] A. Connes, J. Lott, Particle models and noncommutative geometry, Nuclear Physics B18 (1990) supplement 29-47 (1991).

[40] A. Connes, H. Moscovici, The Local Index Formula in Noncommutative Geometry, GAFA 5 (1995), 174-243

[41] J. Dixmier, Existence de traces non normales, (French) C. R. Acad. Sci. Paris 262 (1966) 1107-1108.

[42] J. Dixmier, Les algèbres d'opérateurs dans l'espace Hilbertien (Algèbres de von Neumann), GauthierVillars, Paris, 1969.

[43] P.G. Dodds, T.K. Dodds, B. de Pagter, Non-commutative Banach function spaces, Math. Z. 201 (1989), 583-597. 
[44] P.G. Dodds, T.K. Dodds, B. de Pagter, Fully symmetric operator spaces, Integr. Equat. Oper. Th. 15 (1992), 942-972.

[45] P.G. Dodds, B. de Pagter, F.A. Sedaev, E.M. Semenov, F.A. Sukochev, Singular symmetric functionals, (Russian) Zap. Nauchn. Sem. S.-Peterburg., Otdel. Mat. Inst. Steklov. (POMI) 290 (2002), Issled. po Linein. Oper. i Teor. Funkts. 30, 42-71, 178; translation in J. Math. Sci. (N. Y.) 124, (2004), no. 2, 4867-4885.

[46] P.G. Dodds, B. de Pagter, F.A. Sedaev, E.M. Semenov, F.A. Sukochev, Singular symmetric functionals and Banach limits with additional invariance properties, (Russian) Izv. Ross. Akad. Nauk, Ser. Mat. 67 (2003), no. 6, 111-136.

[47] P. G. Dodds, B. de Pagter, E. M. Semenov, F. A. Sukochev Symmetric functionals and singular traces, Positivity 2 (1998), 47-75.

[48] K. Dykema, T. Figiel, G. Weiss, M. Wodzicki, Commutator structure of operator ideals, Advances Math. 185 (2004), no. 1, 1-79.

[49] K. Dykema, N. Kalton, Sums of commutators in ideals and modules of type II factors, Ann. Inst. Fourier, Grenoble, 55 (2005), 931-971.

[50] K. Dykema, N. Kalton, Spectral characterization of sums of commutators. II, J. Reine Angew. Math. 504 (1998), 127-137.

[51] T. Fack, Sur la notion de valeur caractéristique, J. Operator Theory 7 (1982), 307-333.

[52] T. Fack, Sums of commutators in non-commutative Banach function space, J. Funct. Analysis, 207 (2004), 358-398.

[53] T. Fack, H. Kosaki, Generalised s-numbers of $\tau$-measurable operators, Pacific J. Math., 123 (1986), 269-300.

[54] M. Farber, S. Weinberger, On the zero in the Spectrum Conjecture, Annals of Math., 154 (2001), 139-154.

[55] E. Getzler, The Odd Chern Character in Cyclic Homology and Spectral Flow, Topology, 32 (1993), 489-507.

[56] I. C. Gohberg, M. G. Krein, Introduction to the theory of linear nonselfadjoint operators., Translated from the Russian by A. Feinstein. Translations of Mathematical Monographs, Vol. 18 American Mathematical Society, Providence, R.I. 1969.

[57] J.M. Gracia-Bondia, J.C. Varilly, H. Figueroa, Elements of Noncommutative Geometry, Birkhauser, Boston, 2001.

[58] D. Guido, T. Isola, Singular traces for semifinite von Neumann algebras, J. Funct. Analysis, 134 (1995), 451-485.

[59] D. Guido, T. Isola, On the domain of singular traces, Internat. J. Math., 13 (2002), 667-674.

[60] D. Guido, T. Isola, Singular traces and their applications to geometry, Operator algebras and quantum field theory (Rome, 1996), 440-456, Internat. Press, Cambridge, MA, 1997.

[61] D. Guido, T. Isola, Dimensions and singular traces for spectral triples, with applications to fractals, J. Funct. Analysis, 203 (2003), no. 2, 362-400.

[62] V.W. Guillemin, A new proof of Weyl's formula on the asymptotic distribution of eigenvalues, Advances in Math. 55 (1985) 131-160.

[63] G.H. Hardy, Divergent Series, Oxford, Clarendon Press, 1949.

[64] N. Higson, The Local Index Formula in Noncommutative Geometry, Contemporary developments in algebraic K-theory, 443-536, ICTP Lect. Notes, XV, Abdus Salam Int. Cent. Theoret. Phys., Trieste, 2004.

[65] W. Kalau, Hamilton formalism in non-commutative geometry, J. Geom. Phys. 18 (1996), 349-380.

[66] N. Kalton, Spectral characterization of sums of commutators. I, J. Reine Angew. Math. 504 (1998), $115-125$.

[67] D. Kastler, Noncommutative geometry and basic physics in Geometry and quantum physics Schladming lectures 1999, Lecture Notes in Physics 543 (2000) 131-230 and Noncommutative geometry and fundamental physical interactions J. Math. Phys. 41 (2000) 3867-3891.

[68] N. Kalton, F. Sukochev, Rearrangement invariant functionals with applications to traces on symmetrically normed ideals, Canadian Math. Bull. (to appear).

[69] S.G. Krĕ̌n, Yu.I. Petunīn, E.M Semenov, Interpolyatsiya lineı̆nykh operatorov. (Russian). "Nauka", Moscow, 1978; Interpolation of linear operators. Translated by J. Szücs. Translations of Mathematical Monographs, 54. American Mathematical Society, Providence, R.I., 1982. 
[70] M. Lesch, On the index of the infinitesimal generator of a flow, J. Operator Theory 26 (1991), no. 1, 73-92.

[71] J. Lindenstrauss, L. Tzafriri, Classical Banach spaces. I. Sequence spaces, Results in Mathematics and Related Areas, 92. Springer-Verlag, Berlin-New York, 1977.

[72] J. Lindenstrauss, L. Tzafriri, Classical Banach spaces. II. Function spaces, Results in Mathematics and Related Areas, 97. Springer-Verlag, Berlin-New York, 1979.

[73] J-L Loday, Cyclic Homology, 2nd Ed, Springer-Verlag, Berlin, 1998.

[74] S. Lord, A. Sedaev, F. Sukochev, Dixmier traces as singular symmetric functionals and applications to measurable operators, J. Funct. Analysis 224 (2005), no. 1, 72-106.

[75] G.G. Lorentz, A contribution to the theory of divergent sequences, Acta. Math. 80 (1948), 167-190.

[76] Yu. I. Manin Algebraic aspects of non-linear differential equations J. Soviet Math 11 (1979), 1-122.

[77] V. Mathai, Spectral flow, eta invariants and von Neumann algebras, J. Funct. Analysis, 109 (1992), 442-456.

[78] V.S. Perera, Real Valued Spectral Flow in Type $I I_{\infty}$ Factor, Ph.D. Thesis, IUPUI, 1993 and Realvalued spectral flow. Multivariable operator theory, 307-318, Contemp. Math., 185, Amer. Math. Soc., Providence, RI, 1995.

[79] V.S. Perera, Real Valued Spectral Flow in a Type $I I_{\infty}$ Factor, Houston J. Math., 25 (1999), 55-66.

[80] J. Phillips, Self-Adjoint Fredholm Operators and Spectral Flow, Canad. Math. Bull., 39(1996), 460467.

[81] J. Phillips, Spectral Flow in Type I and Type II factors - a New Approach, Cyclic cohomology and noncommutative geometry (Waterloo, ON, 1995), 137-153, Fields Inst. Commun., 17, Amer. Math. Soc., Providence, RI, 1997.

[82] J. Phillips, I. F. Raeburn An Index Theorem for Toeplitz Operators with Noncommutative Symbol Space, J. Funct. Analysis, 120 (1993) 239-263.

[83] R. Prinzis, Traces Residuelles et Asymptotique du Spectre d'Operateurs Pseudo-Differentiels Thèse, Université de Lyon, unpublished.

[84] M. Reed, B. Sumon, Methods of modern mathematical physics, I. Functional analysis. Second edition. Academic Press, Inc., New York, 1980.

[85] A. Rennie, Smoothness and Locality for Nonunital Spectral Triples K Theory 28 (2003), 127-161.

[86] W. Rudin, Principles of Mathematical Analysis, $3^{\text {rd }}$ McGraw-Hill, New York, London, Toronto, 1976.

[87] E. Schrohe, M. Walze, J.-M. Warzecha, Construction de triplets spectraux partir de modules de Fredholm, C. R. Acad. Sci. Paris Sér. I Math. 326 (1998), no. 10, 1195-1199.

[88] M.A. Shubin, Spectral theory and index of elliptic operators with almost periodic coefficients, (Russian) Uspekhi Mat. Nauk 34 (1979), no. 2(206), 95-135. English translation: Russian Math. Surveys 34 (1979), no. 2, 109-157.

[89] M.A. Shubin, Almost periodic pseudodifferential operators, and von Neumann Algebras, (Russian), Trudy Moskov. Mat. Obšc. 35 (1976), 103-164. English Translation: Moscow Math. Soc. 1 (1979), 103-166.

[90] L. Sucheston, Banach limits, Amer. Math. Monthly 74 (1967), 308-311.

[91] F. A. Sukochev, Operator estimates for Fredholm Modules, Canad.J.Math. 52 (2000), 849-896.

[92] J.V. Varga, Traces on irregular ideals, Proc. Amer. Math. Soc. 107 (1989), 715-723.

[93] M. Wodzicki, Noncommutative residue. I. Fundamentals K-theory, arithmetic and geometry (Moscow, 1984-1986), 320-399, Lecture Notes in Math., 1289, Springer, Berlin, 1987. 